\title{
A SEARCH FOR A NEW GAUGE BOSON $A^{\prime}$
}

\author{
Eric Jensen \\ Herndon, Virginia \\ Master of Science, College of William and Mary, 2010 \\ Bachelor of Arts, Christopher Newport University, 2008
}

A Dissertation presented to the Graduate Faculty of the College of William and Mary in Candidacy for the Degree of Doctor of Philosophy

Department of Physics

The College of William and Mary

August 2013 
(C) 2013

Eric Jensen

All rights reserved. 


\section{APPROVAL PAGE}

This Dissertation is submitted in partial fulfillment of the requirements for the degree of

Doctor of Philosophy

Eric Jensen

Approved by the Committee, May, 2013

Professor David Armstrong, Physics

The College of William and Mary

Professor Carl Carlson, Physics

The College of William and Mary

Professor Harvey Thinker, Physics

The College of William and Mary

Dr. Bogdan Wojtsekhowski, Physics

Science Fiction Institute 


\begin{abstract}
PAGE
In the Standard Model, gauge bosons mediate the strong, weak, and electromagnetic forces. New forces could have escaped detection only if their mediators are either heavier than $\mathcal{O}(\mathrm{TeV})$ or weakly coupled to charged matter. New vector bosons with small coupling $\alpha^{\prime}$ arise naturally from a small kinetic mixing with the photon and have received considerable attention as an explanation of various dark matter related anomolies. Such particles can be produced in electron-nucleus fixed-target scattering and then decay to $e^{+} e^{-}$pairs. New light vector bosons and their associated forces are a common feature of Standard Model extensions, but existing constraints are remarkably sparse.

The APEX experiment will search for a new vector boson $A^{\prime}$ with coupling $\alpha^{\prime} / \alpha_{\mathrm{fs}} \gtrsim 6 \times 10^{-8}$ to electrons in the mass range $65 \mathrm{MeV}<m_{A^{\prime}}<550 \mathrm{MeV}$. The experiment will study $e^{+} e^{-}$production off an electron beam incident on a high-Z target in Hall A at Jefferson Lab. The $e^{-}$and $e^{+}$will be detected in the High Resolution Spectrometers (HRSs). The invariant mass spectrum of the $e^{+} e^{-}$pairs will be scanned for a narrow resonance corresponding to the mass of the $A^{\prime}$.

A test run for the APEX experiment was held in the summer of 2010. Using the test run data, an $A^{\prime}$ search was performed in the mass range $175-250 \mathrm{MeV}$. The search found no evidence for an $A^{\prime} \rightarrow e^{+} e^{-}$reaction, and set an upper limit of $\alpha^{\prime} / \alpha_{\mathrm{fs}} \simeq 10^{-6}$.
\end{abstract}


TABLE OF CONTENTS

List of Tables $\ldots \ldots \ldots \ldots \ldots \ldots$ ii

List of Figures . . . . . . . . . . . . . . . iii

CHAPTER

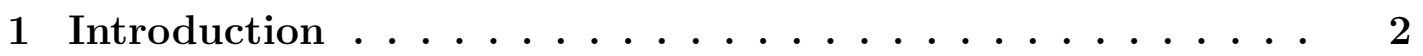

1.1 Dark Matter . . . . . . . . . . . . . . . . . . . . . . . 2

1.2 Experimental Overview . . . . . . . . . . . . . . . . 2

2 Motivation for the Existence of the $A^{\prime} \ldots \ldots \ldots \ldots$

2.1 Standard Model Extensions $\ldots \ldots \ldots$

2.2 Super Symmetry $\ldots \ldots \ldots \ldots$

2.3 Grand Unified Theory $\ldots \ldots \ldots \ldots$

2.4 Dark Matter Related Anomalies . . . . . . . . . . . . . . . 3

$2.5 \quad$ Current Limits on Light $U 1$ Gauge Bosons $\ldots \ldots \ldots$

3 Jefferson Lab. . . . . . . . . . . . . . . . . 4

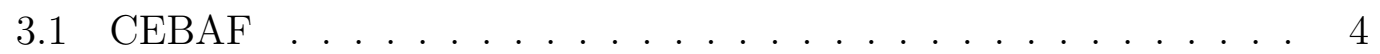

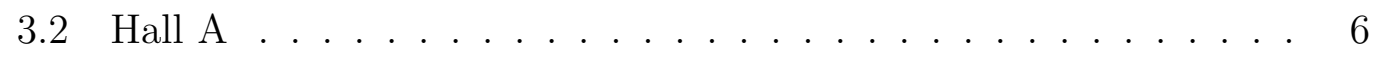

3.2 .1 Beamline. . . . . . . . . . . . . . . . . . 7

$3.2 .2 \quad$ High Resolution Spectrometers $\ldots \ldots \ldots$

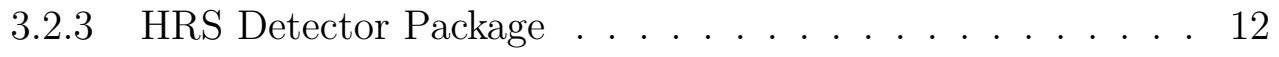

$3.2 .4 \quad$ Data Acquisition $\ldots \ldots \ldots \ldots$ 
4 The APEX Experiment $\ldots \ldots \ldots \ldots \ldots \ldots \ldots$

$4.1 \quad$ A' Production in Fixed Target Experiments $\ldots . . . . . . . .21$

4.2 Signal and Trident Kinematics _ . . . . . . . . . . . . . . . . 24

4.3 Accidentals and rates . . . . . . . . . . . . . . . . 27

$4.4 \quad$ Experimental Setup . . . . . . . . . . . . . . . . . . . . . 30

4.4 .1 Target $\ldots \ldots \ldots \ldots \ldots \ldots \ldots$

4.4 .2 Septum Magnet . . . . . . . . . . . . . . . . . . . . . 34

4.4 .3 Track Measurement . . . . . . . . . . . . . . . . . . . . 34

$4.4 .4 \quad$ SciFi Detector . . . . . . . . . . . . . . . . . 36

4.4 .5 Particle Identification . . . . . . . . . . . . . . . . . 37

$4.4 .6 \quad$ Trigger and DAQ $\ldots \ldots \ldots \ldots \ldots \ldots$

4.5 Proposed Measurement . . . . . . . . . . . . . . . . . . . . . . . 41

5 The APEX Test Run . . . . . . . . . . . . . . . 42

5.1 PAC Concerns . . . . . . . . . . . . . . . . . . . . . 42

$5.2 \quad$ Experimental Setup . . . . . . . . . . . . . . . . . 43

$5.2 .1 \quad$ Targets $\ldots \ldots \ldots \ldots \ldots \ldots \ldots \ldots \ldots$

5.2 .2 Septum and Beam Steering Magnets . . . . . . . . . . . 45

$5.2 .3 \quad$ Electronics and Trigger . . . . . . . . . . . . . . . 46

6 Detector Calibrations . . . . . . . . . . . . . . 49

6.1 HRS Detectors $\ldots \ldots \ldots \ldots \ldots \ldots$

6.1 .1 Scintillators . . . . . . . . . . . . . . . 50

6.1 .2 Gas Cherenkov Detector . . . . . . . . . . . . . . . 52

6.1 .3 Drift Chambers . . . . . . . . . . . . . . . . . . 52

6.2 Beam Position . . . . . . . . . . . . . . . . . . . . . . 55

6.3 Optics Calibration $\ldots \ldots \ldots \ldots \ldots \ldots \ldots$

$6.3 .1 \quad$ Coordinate Systems . . . . . . . . . . . . . . . . 58 
$6.3 .2 \quad$ General Approach . . . . . . . . . . . . . . . . . . . . . . 59

6.3 .3 Procedure . . . . . . . . . . . . . . . . . . . . . . 61

6.3 .4 Results . . . . . . . . . . . . . . . . . . . . . . 64

7 Data Analysis $\ldots \ldots \ldots \ldots \ldots \ldots$

7.1 Analysis software $\ldots \ldots \ldots \ldots \ldots$

7.2 Particle identification $\ldots \ldots \ldots \ldots \ldots$. . . . . . . . 70

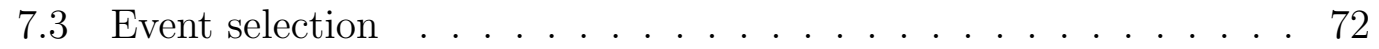

$7.3 .1 \quad$ Coincidence timing $\ldots \ldots \ldots \ldots \ldots$

7.3 .2 Acceptance cuts . . . . . . . . . . . . . . 74

$7.3 .3 \quad$ Final event sample . . . . . . . . . . . . . . 76

7.4 Track reconstruction $\ldots \ldots \ldots \ldots \ldots \ldots$

7.5 Invariant mass calculation and comparison to MC data . . . . . . 81

7.6 Mass resolution . . . . . . . . . . . . . . . . . . . . . . . . 84

7.6 .1 Angular resolution . . . . . . . . . . . . . . . . 85

7.6 .2 Determining the mass resolution . . . . . . . . . . 88

7.7 Addressing the PAC concerns $\ldots \ldots \ldots$. . . . . . . . . . 89

8 Resonance Search and Results . . . . . . . . . . . . . 98

8.1 Searching for a resonance $\ldots \ldots \ldots \ldots \ldots$

$8.1 .1 \quad$ Pseudo-data sets . . . . . . . . . . . . . . . 100

8.1 .2 Profile likelihood ratio . . . . . . . . . . . . . . . 100

8.1 .3 The Look Elsewhere Effect . . . . . . . . . . . . . . . . . 102

$8.1 .4 \quad$ Search parameters . . . . . . . . . . . . . . . . . 104

8.1 .5 Results . . . . . . . . . . . . . . . . . . . . . . 108

$8.2 \quad$ Setting limits on $\alpha^{\prime} / \alpha_{\mathrm{fs}} \ldots \ldots \ldots \ldots \ldots \ldots \ldots$

$8.2 .1 \quad$ Setting limits on $S \ldots \ldots \ldots \ldots$

8.2 .2 Results . . . . . . . . . . . . . . . . . . . . . 110 


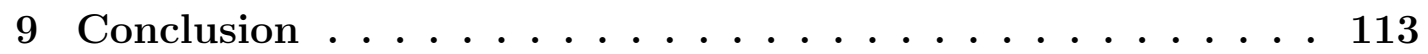

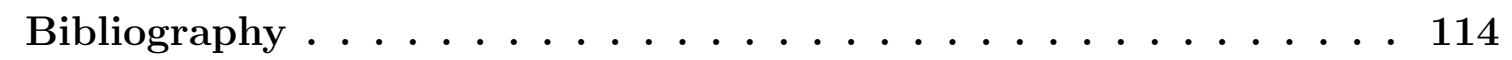




\section{LIST OF TABLES}

Table

Page

3.1 Main design characteristics of the Hall A high resolution spectrometers. The resolution values are for the FWHM. . . . . . . . . . . . . . 13

4.1 Expected rates . . . . . . . . . . . . . . . . . . . 30

5.1 Triggers of the APEX test run. . . . . . . . . . . . . . . . . . . . 48

6.1 Summary of the angular calibration runs . . . . . . . . . . . 65

$7.1 \quad$ Loose cuts for track determining reconstruction efficiency . . . . . . . 80

7.2 Summary of the contributions to the angular resolution. . . . . . . . 87 


\section{LIST OF FIGURES}

Figure

Page

3.1 The CEBAF accelerator layout for $6 \mathrm{GeV}$ operation . . . . . . . . . . 5

3.2 Top view of Hall $\mathrm{A}$. . . . . . . . . . . . . . . . . . . . . . . . . . . . . . . 7

3.3 The beam position monitors . . . . . . . . . . . . . . . . . . . . . . . 9

3.4 Rastered beam spot . . . . . . . . . . . . . . . . . . . . . . 10

3.5 Layout of the HRS . . . . . . . . . . . . . . . . . . . . . . . 12

3.6 Side view of the detector stack . . . . . . . . . . . . . . . . . . . . . . 14

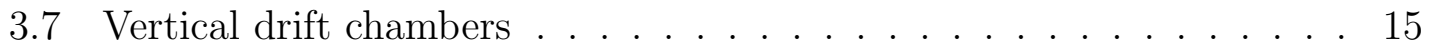

3.8 S2m scintillator plane . . . . . . . . . . . . . . . . . . . . . . 16

3.9 Gas Cherenkov counter . . . . . . . . . . . . . . . . . . . . . . . . . . 17

3.10 Lead glass calorimeters . . . . . . . . . . . . . . . . . 18

$4.1 \quad A^{\prime}$ production off a fixed target . . . . . . . . . . . . . . . . 22

4.2 Diagrams of the QED background processes . . . . . . . . . . . . . 25

4.3 Electron momentum vs. positron momentum simulation . . . . . . . 26

4.4 Anticipated sensitivity for APEX . . . . . . . . . . . . . . . . 28

4.5 The layout of the experimental setup . . . . . . . . . . . . . . . . . . . . . . . . 31

4.6 Final design of the APEX target. . . . . . . . . . . . . . . . . . . . . 33

4.7 SciFi detector . . . . . . . . . . . . . . . . . . . . . . 38

4.8 APEX trigger . . . . . . . . . . . . . . . . . . . . . . . . . . 39

4.9 Sparsification timing diagram . . . . . . . . . . . . . . . . . 40

5.1 The lead target . . . . . . . . . . . . . . . . . . . 45

5.2 The septum magnets . . . . . . . . . . . . . . . . . . . 46

5.3 Trigger electronics for the test run . . . . . . . . . . . . . . . . . . 48

6.1 Timing alignment of the S2m plane . . . . . . . . . . . . . . . . . 51 
6.2 Timing alignment of the S2m plane . . . . . . . . . . . . . . . . . 53

6.3 VDC time offset calibration. . . . . . . . . . . . . . . . . . . . . . . . 54

6.4 Raster current vs. BPM position . . . . . . . . . . . . . . 56

6.5 Coordinate systems . . . . . . . . . . . . . . . . . 6 60

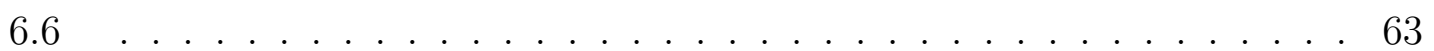

6.7 Schematic of sieve slit setup . . . . . . . . . . . . . . . . 63

6.8 Reconstructed foil positions . . . . . . . . . . . . . . . . . . 64

6.9 Reconstructed sieve holes after calibration . . . . . . . . . . . . . 67

6.10 Results of $\delta$ calibration . . . . . . . . . . . . . . . . . . . . 68

7.1 Particle identification . . . . . . . . . . . . . . . . . . . . . 72

7.2 Coincidence timing spectrum . . . . . . . . . . . . . . . . . . . 74

7.3 PREX collimator . . . . . . . . . . . . . . . . . . . . . 75

7.4 PREX collimator . . . . . . . . . . . . . . . . . 76

7.5 VDC cluster example . . . . . . . . . . . . . . . . . . . . . . . . . . . 78

7.6 VDC track reconstruction $\ldots \ldots \ldots$. . . . . . . . . . . . . . . . . . 79

7.7 Tracking modifications . . . . . . . . . . . . . . . . . . 80

7.8 Loose cuts for determining track reconstruction efficiency . . . . . . . . 81

7.9 Invariant mass spectrum of the test run data . . . . . . . . . . . . . 83

7.10 Comparison of the test run data and calculated invariant mass spec-

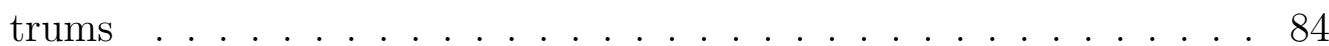

7.11 Comparison of the test run data and calculated angular distributions 85

7.12 1D reconstructed spatial distributions of a sieve hole. . . . . . . . . . 88

7.13 Difference between simulated and real invariant mass . . . . . . . . . 90

7.14 Mass resolution as a function of invariant mass . . . . . . . . . . . . . 91

7.15 VDC wire efficiency . . . . . . . . . . . . . . . . . 93

7.16 VDC drift time for high rate data . . . . . . . . . . . . . . . . . . 94

7.17 VDC drift time-to-distance calibration . . . . . . . . . . . . . . . . . 95

7.18 VDC cluster size . . . . . . . . . . . . . . . . . . . . . . . 96

7.19 PID at high rates . . . . . . . . . . . . . . . . . 97

8.1 Bell curve . . . . . . . . . . . . . . . . . . . 100

8.2 Toy function . . . . . . . . . . . . . . . . . . . . . . 101

8.3 Relation of $p$-value to the test statistic $t$ and significance $Z$. . . . . 103

8.4 Setting a limit on the observed $p$-value . . . . . . . . . . . . . . . 105

8.5 Pulls generated from different bin sizes . . . . . . . . . . . . . . . . 106

8.6 Window size and polynomial order . . . . . . . . . . . . . . . . 107

8.7 Local $p$-values and the upper bound on number of signal events . . . 111 


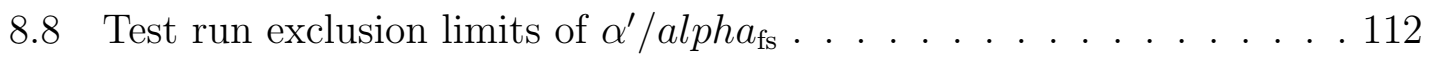


A SEARCH FOR A NEW GAUGE BOSON $A^{\prime}$ 


\title{
CHAPTER 1
}

\section{Introduction}

\author{
$1.1 \quad$ Dark Matter
}

1.2 Experimental Overview 


\section{CHAPTER 2}

Motivation for the Existence of

the $A^{\prime}$

2.1 Standard Model Extensions

2.2 Super Symmetry

2.3 Grand Unified Theory

2.4 Dark Matter Related Anomalies

2.5 Current Limits on Light $U 1$ Gauge Bosons 


\section{CHAPTER 3}

\section{Jefferson Lab}

The APEX experiment will run in Hall A of Thomas Jefferson National Accelerator Facility (Jefferson Lab, or JLab). Jefferson Lab is a U.S. national laboratory used to study the structure of nuclear matter. The laboratory's main research tool is the Continuous Electron Beam Accelerator Facility (CEBAF). The CEBAF accelerator uses superconducting radio frequency cavities to accelerate electrons to energies up to $5.7 \mathrm{GeV}$. The electron beam is delivered to three different experimental halls where it interacts with stationary targets. Each hall can be equipped with unique spectrometers and detectors that allow physicists to study these interactions.

This chapter will describe the details of the accelerator and experimental Hall A at Jefferson Lab. The details of the APEX experimental setup will be discussed in chapter 4 .

\subsection{CEBAF}

The Continuous Electron Beam Accelerator Facility (CEBAF) is a medium energy electron accelerator capable of delivering continuous beams of electrons with energies up to $5.7 \mathrm{GeV}$ and currents up to $200 \mu \mathrm{A}$. The energy and high current of 


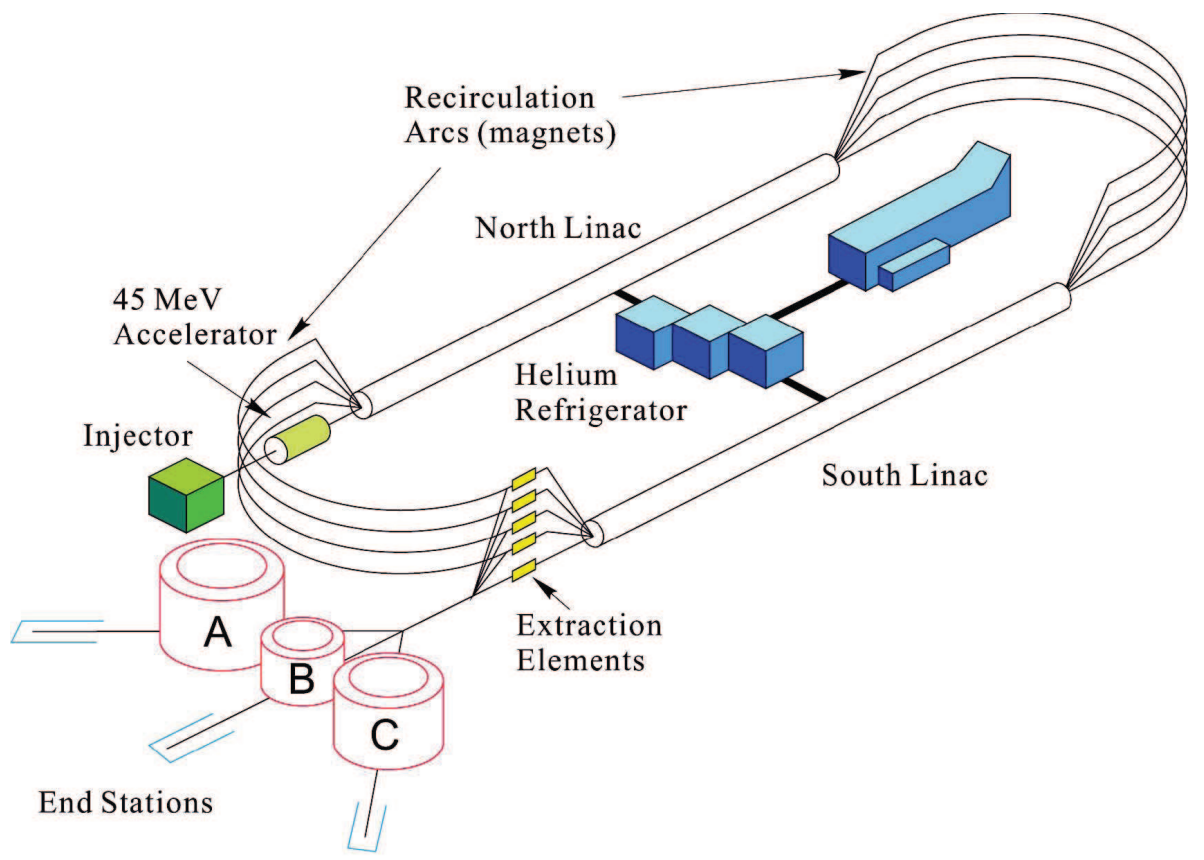

FIG. 3.1: The CEBAF accelerator layout for $6 \mathrm{GeV}$ operation. The electrons are produced in the injector and then accelerated to $45 \mathrm{MeV}$ before entering the main accelerator ring. The electrons can then be accelerated to up to $5.7 \mathrm{GeV}$ by recirculating them five times through two linear accelerators. After the electrons reach the desired energy, the beam is simultaneously delivered to all three experiemental halls.

this electron beam allow us to search for the $A^{\prime}$ within a particularly well motivated region of parameter space.

The CEBAF configuration consists of a polarized electron source, an injector, two linear accelerators (linacs), two sets of recirculating magnetic arcs, and extraction elements. The accelerator is capable of delivering continuous wave electron beams to three experimental halls simultaneously (Fig. 3.1).

The initial electrons are created in the injector by illuminating a single photocathode with three interlaced RF-gain-switched lasers to produce $100 \mathrm{keV}$ electrons through a process analogous to the photoelectric effect and an electric field gradient. The injector provides separate beams to the three experimental halls by interlacing light from three diode lasers pulsed at $499 \mathrm{MHz}$ onto a strained GaAs photocathode. Separated by $120^{\circ}$ of $\mathrm{rf}$ space, the three beams form a $1497 \mathrm{MHz}$ train of electrons. 
After the electrons are produced, they are accelerated to $5 \mathrm{MeV}$ in a cryounit (a single pair of superconducting cavities) and then accelerated to $45 \mathrm{MeV}$ in two cryomodules (four cryounits per cryomodule). These electrons are then injected into the main accelerator where they can accelerate to higher energies.

The main accelerator features a pair of superconducting radio frequency linacs that accelerate the electrons until they reach the desired energy. Each linac consists of 160 superconducting cavities housed by 20 cryomodules. After injection, the electrons pass through the first linac and gain about $600 \mathrm{MeV}$ of energy before passing through the first recirculation arc. After passing through the second linac, the "one pass" electrons can either be delivered to one of the experimental halls, or recirculate around the accelerator loop to acquire more energy and become a higher "pass" beam. The electrons can be recirculated a maximum of five times before being sent to one of the experimental halls [1].

Liquid helium is used to keep the superconducting cavities at a temperature of $2 \mathrm{~K}$. The liquid helium is produced at the Central Helium Liquifier (CHL) located on site.

The properties of the beam can be monitored through the EPICS data system. Furthermore, a $499 \mathrm{MHz}$ phase-locked clock is used to generate a signal for every electron bundle. This signal is sent to the CEBAF Online Data Acquisition (CODA) system for each event so that events in the detectors can be associated with a specific bundle.

\section{$3.2 \quad$ Hall A}

Hall $\mathrm{A}$ is the largest of the three experimental halls at Jefferson Lab. It has a circular shape measuring $174 \mathrm{ft}$ in diameter and is $80 \mathrm{ft}$ from floor to ceiling. The floor of the hall is located $35 \mathrm{ft}$ below ground, and the entire hall is well 


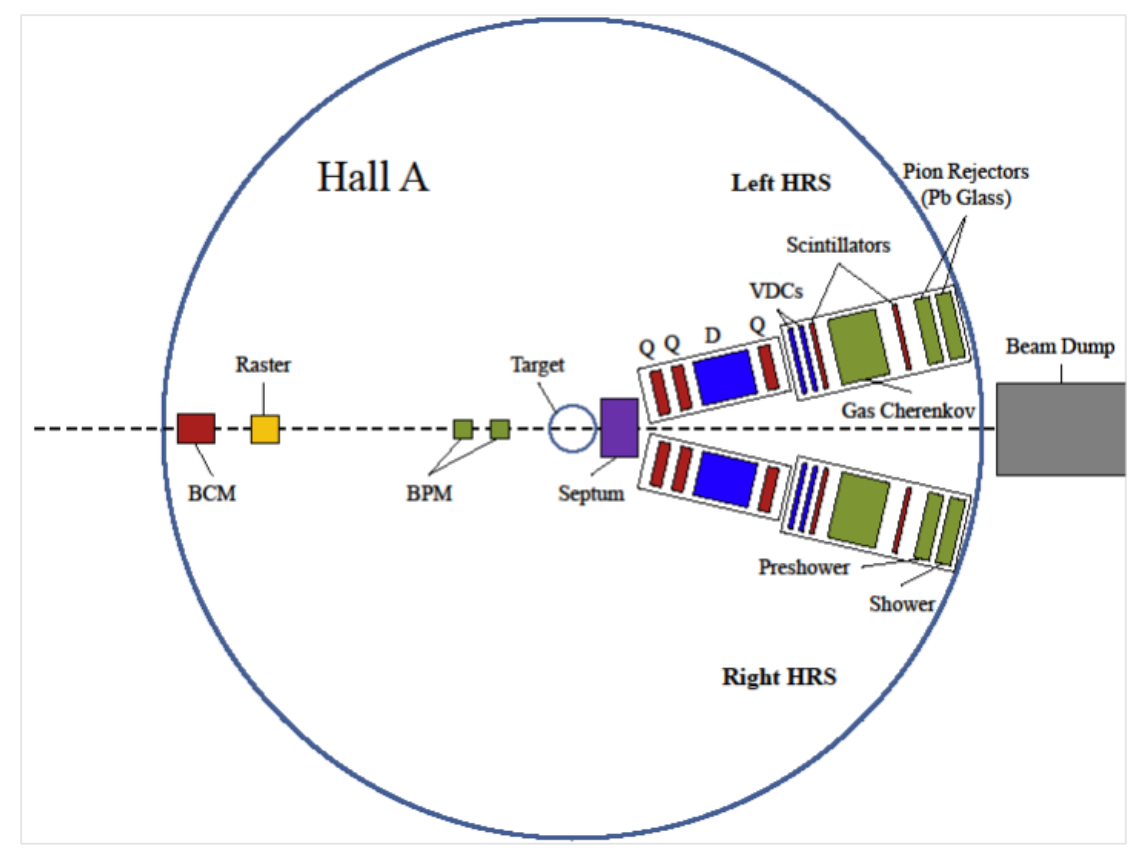

FIG. 3.2: Top view schematic of Hall A. The electron beam enters the hall from the left, then passes several beam quality monitors before hitting the target at the center of the hall. Two High Resolution Spectrometers are used to detect the products of the electron-target interaction.

shielded with concrete and a thick layer of earth to contain radiation and reduce cosmic background radiation. The basic layout of Hall A is shown in Fig. 3.2. The experimental hall features two High Resolution Spectrometers (HRSs) that pivot around a target located at the center of the hall.

\subsubsection{Beamline}

The Hall A beamline carries the beam from the beam switch yard to the target. Electrons that do not interact with the target continue along the beamline to a well shielded, isolated beam dump. Along the beamline are several components used to 
monitor certain properties of the beam. Some of the properties measured include the beam's current, position, and direction. In this section, we will describe the components along the beamline that are relevant to the APEX Experiment.

\section{Beam Current Monitors}

The beam current monitor (BCM) in Hall A is used to measure the current and integrated charge of the electron beam over a period of time. It consists of two RF cavities tuned to the frequency of the beam $(1497 \mathrm{MHz})$, resulting in voltage levels at their outputs that are proportional to the beam current. The two RF cavities sandwich an Unser monitor, which is used as an absolute reference for calibration [2]. In addition, instrumentation at the injector section of the accelerator provides a reference for calibration. The cavities and Unser monitor are enclosed in a temperature-stabilized box located $25 \mathrm{~m}$ upstream of the target.

Each of the RF output signals from the two cavities is split into two parts: sampled and integrated data. The sampled data is sent to a high-precision voltmeter. The voltmeter provides an output representing the RMS value of the input signal to the EPICS data stream every 1-2 seconds. The integrated data is sent to an RMS to DC converter, and then to a Voltage-To-Frequency (VTOF) converter whose output frequency is proportional to the input voltage level. The output signals are fed to $200 \mathrm{MHz}$ scalers, which accumulate during the run. At the end of the run, the scalers give a number proportional to the time-integrated voltage and therefore accurately record the integrated current, i.e., the total beam charge. The output of the RMS to DC converter is linear for currents of $5 \mu \mathrm{A}$ to $200 \mu \mathrm{A}$. A set of amplifiers with gain factors of 1,3 , and 10 is used to extend the linear region to lower currents at the expense of saturating at high currents. Hence there are three signals coming from each BCM, giving six total signals going to the scalers of each spectrometer to provide beam charge data for each run. A BCM calibration 


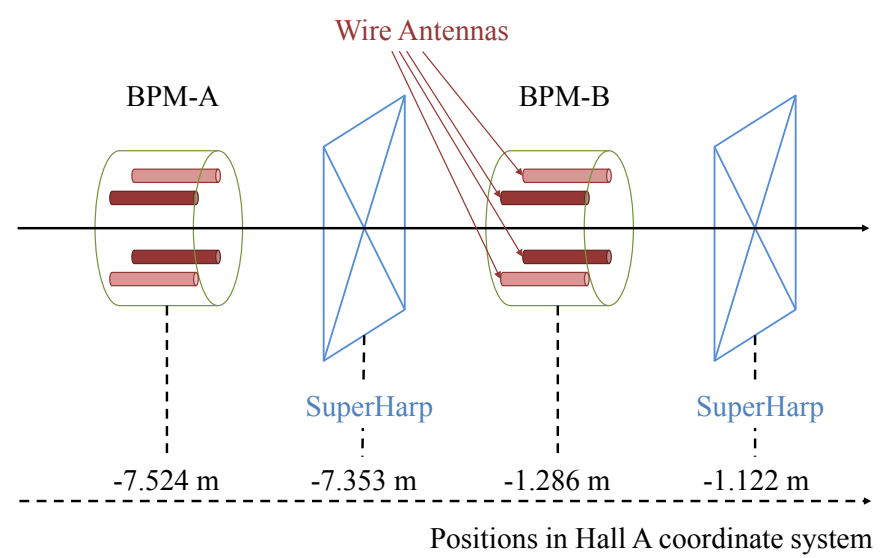

FIG. 3.3: The beam position monitors are located upstream of the target. Each BPM contains four antennas. The relative beam position is determined using the difference-over-sum technique between two opposite antennas [4]. To determine the absolute position, the BPMs are calibrated against the SuperHarps.

is typically performed every 2-3 months, allowing the accumulated charge to be determined with an accuracy of $\pm 0.5 \%[3]$.

\section{Beam Position Monitors}

Two beam position monitors (BPMs), located $7.524 \mathrm{~m}$ and $1.286 \mathrm{~m}$ upstream of the target (see Fig. 3.2.1), are used to determine the position and direction of the beam at the target. Each BPM contains four antennas surrounding the beamline. When the beam passes through the BPM, it induces signals on the antennas that can be measured and used to determine the position of the beam to within $100 \mu \mathrm{m}$ for currents above $1 \mu \mathrm{A}$. These BPMs provide non-destructive determination of the position and direction of the beam at the target location.

The BPMs are calibrated with respect to a set of wire scanners known as SuperHarps, which are located $7.353 \mathrm{~m}$ and $1.122 \mathrm{~m}$ upstream of the target. The positions of the SuperHarps are regularly surveyed with respect to the Hall A coordinates with the accuracy of better than $200 \mu \mathrm{m}$. 


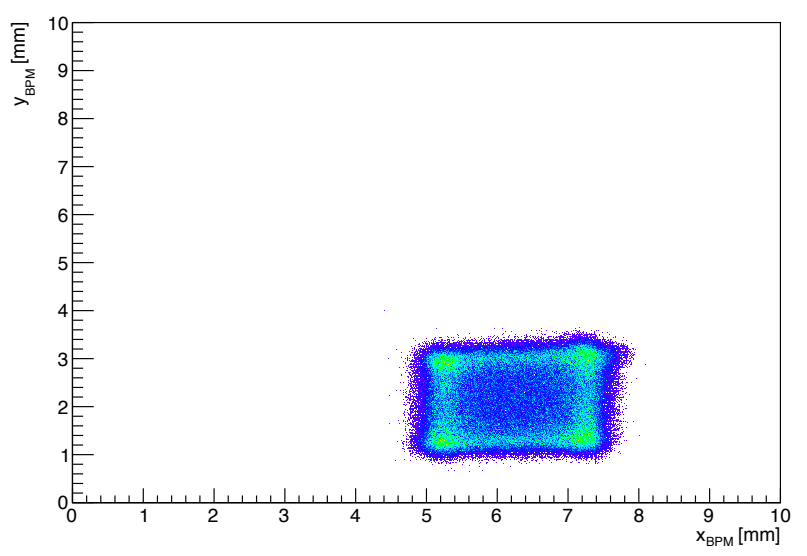

FIG. 3.4: Reconstructed beam spot determined by the BPMs for a $2.5 \mathrm{x} 2.5 \mathrm{~mm}^{2}$ raster size.

For each event information from the BPMs is recorded into the CODA data stream, for each of the 8 antennas. In addition, the beam position averaged over a $0.3 \mathrm{~s}$ interval is logged into the EPICS datastream once every second.

\section{Raster}

If the beam position at the target were fixed at a single point, the target material would heat up to extremely high temperatures, causing severe damage to the target. A set of fast rastering field coils is used to produce small deviations in the beam position at the target in order to avoid overheating the target material. The fast rastering system, located $23 \mathrm{~m}$ upstream of the target, produces deviations of several $\mathrm{mm}$ in both directions at the target location. It is able to sweep across the range at a rate of 17 to $24 \mathrm{kHz}$. The current supplied to the raster is recorded into the CODA data stream for each event to be used to accurately determine the beam position at the target. Fig. 3.4 illustrates an example of a reconstructed beam spot using a $2.5 \times 2.5 \mathrm{~mm}^{2}$ raster size. 


\section{Beam Dump}

After passing the target, the exiting electron beam travels down a thin-walled aluminum spiral corrugated pipe towards the beam dump. The end of the corrugated pipe is connected to a beam diffuser which diffuses the beam over the beam dump surface. The beam dump is designed to operate at a maximum beam power of 900 $\mathrm{kW}$ and $190 \mu \mathrm{A}$.

\subsubsection{High Resolution Spectrometers}

Hall A boasts a pair of $4 \mathrm{GeV} / c$ superconducting High Resolution Spectrometers (HRSs), which are nominally identical in terms of their magnetic properties. The vertical bending design includes two $\cos \theta$ quadrupoles followed by a dispersive dipole. Following the dipole is a third $\cos \theta$ quadrupole. The (QQDQ) configuration is used to deflect charged particles $45^{\circ}$ upward toward the detector hut (Fig. 3.5). All magnets are superconducting and have independent cryogenic controls and reservoirs.

The main purpose of the HRSs is to measure the momentum of particles scattered from the target. The momentum of a charged particle is determined by analyzing the trajectory of the particle through the spectrometer. When charged particles enter the spectrometer, the dipole magnet bends the particles vertically upward. The angle at which the particle bends depends on the particle's momentum. Vertical drift chambers are used to measure the position and angle of incoming particles. This information is used to reconstruct the particle's trajectory back to the target. The momentum of the particle can then be calculated using information from the reconstructed track and the magnetic setting of the spectrometer. More details on the magnetic optics of the HRSs will be discussed in Sec. 6.3.

The HRSs were designed to provide a high momentum resolution, large accep- 


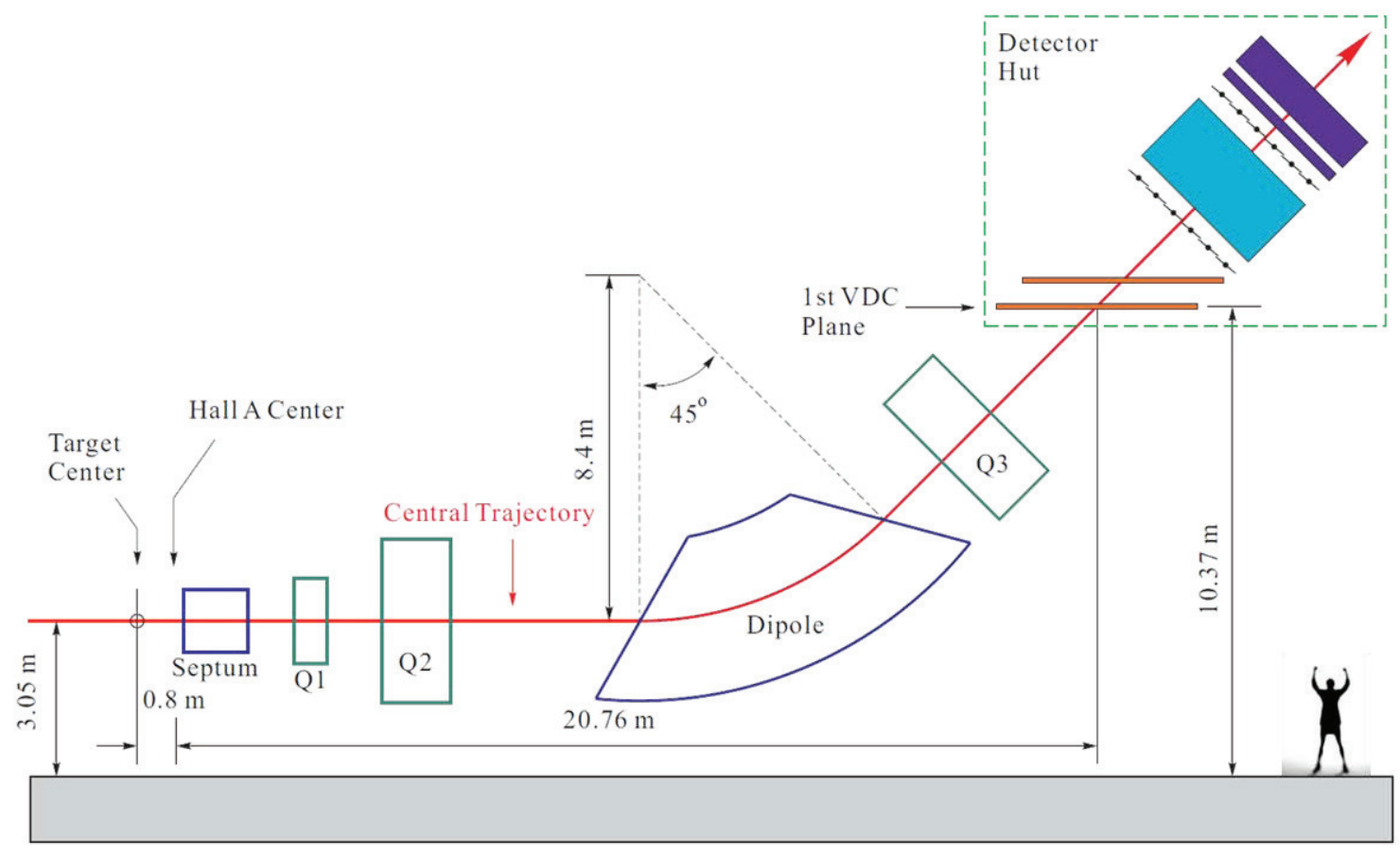

FIG. 3.5: Schematic layout of the HRS. The septum dipole magnet bends charged particles with small scattering angles toward the spectrometer. Two quadrupole magnets focus the incoming particles into the dispersive dipole magnet. The dipole magnet bends the charged particles $45^{\circ}$ upward, and the final quadrupole magnet focuses the particles into the detector hut.

tance in both angle and momentum, good position and angular resolution in the scattering plane, an extended target acceptance, and a large angular range. The momentum resolution of the HRSs is better than $2 \times 10^{-4}$ over a 0.4 to $4.0 \mathrm{GeV} / c$ momentum range. The momentum acceptance is $\pm 4.5 \%$ with an angular acceptance of $\pm 30 \mathrm{mrad}$ horizontal and $\pm 60 \mathrm{mrad}$ vertical. Table 3.1 summarizes the general characteristics of the HRSs. More details can be found in [3].

\subsubsection{HRS Detector Package}

The HRS detector package is designed to perform various measurements of charged particles passing through the spectrometer. The detectors provide a trigger to activate the data acquisition electronics, collect tracking information (position 
TABLE 3.1: Main design characteristics of the Hall A high resolution spectrometers. The resolution values are for the FWHM.

\begin{tabular}{ll}
\hline \hline Configuration & QQDQ vertical bend \\
Bending angle & $45^{\circ}$ \\
Optical length & $23.4 \mathrm{~m}$ \\
Momentum range & $0.3-4.0 \mathrm{GeV} / c$ \\
Momentum acceptance & $-4.5 \%<\delta p / p<+4.5 \%$ \\
Momentum resolution & $1 \times 10^{-4}$ \\
Angular range & \\
$\quad$ Left HRS & $12.5-150^{\circ}$ \\
$\quad$ Right HRS & $12.5-130^{\circ}$ \\
Angular acceptance & \\
$\quad$ Horizontal & $\pm 30 \mathrm{mrad}$ \\
$\quad$ Vertical & $\pm 60 \mathrm{mrad}$ \\
$\quad$ Horizontal & \\
Vertical & $0.5 \mathrm{mrad}$ \\
Solid angle at $\delta p / p=0, y_{0}=0$ & $1.0 \mathrm{mrad}$ \\
\hline \hline
\end{tabular}

and direction), precisely measure the timing for time-of-flight measurements and coincidence determination, and identify the scattered particles. The detector package and all data-acquisition (DAQ) electronics are housed within a shielding hut for protection from radiation. Each detector package is composed of a pair of vertical drift chambers (VDCs), scintillator planes, Cherenkov detectors, and electromagnetic calorimeters (Fig. 3.6).

\section{Vertical Drift Chambers}

A pair of Vertical Drift Chambers (VDCs) provides precise $( \pm 125 \mu \mathrm{m})$ particle tracking information for each HRS. The lower VDC is positioned to coincide as closely as possible with the focal plane of the HRS, and the second VDC is positioned right above it to provide precise angular reconstruction of particle trajectories. Each VDC chamber contains two wire planes separated by about $335 \mathrm{~mm}$. The wires of each successive plane are oriented at $90^{\circ}$ to one another and lie within the laboratory 


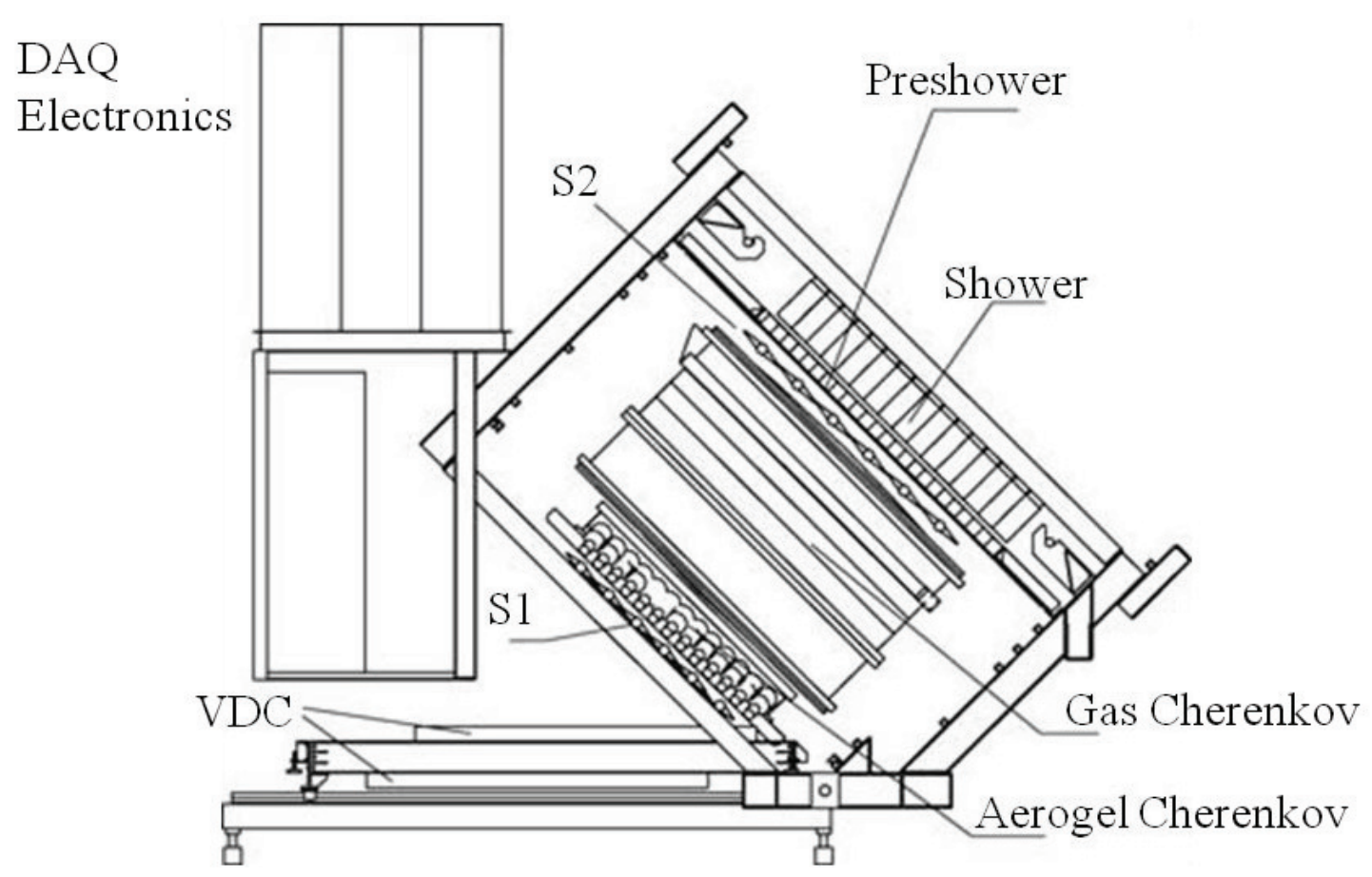

FIG. 3.6: Side view of the detector stack. The relevant detectors for the APEX test run are the VDCs, S2m plane, gas Cherenkov, and electromagnetic calorimeter.

horizontal plane. The wires are oriented at an angle of $45^{\circ}$ with respect to the dispersive and non-dispersive directions, as shown in Fig. 3.7. Each plane consists of 368 wires, spaced $4.24 \mathrm{~mm}$ apart from one another [5].

The VDC chamber is filled with a gas mixture of argon (62\%) and ethane (38\%). The VDCs normally operate with their cathode planes at $-4 \mathrm{kV}$, but for the APEX test run the planes were set to $-3.5 \mathrm{kV}$ due to the expected high rates. Custom made discriminator cards were also installed to allow the VDCs to operate at very high rates.

As charged particles pass through the gas in the chamber they ionize the gas. These ions drift along the electric field lines defined by the cathode planes and are collected on the signal wires in the form of an analog pulse. The pulses are then amplified, discriminated, and used to start multihit Time-to-Digital converters (TDCs) operating in common stop mode. The TDC data is read out to the CODA data 


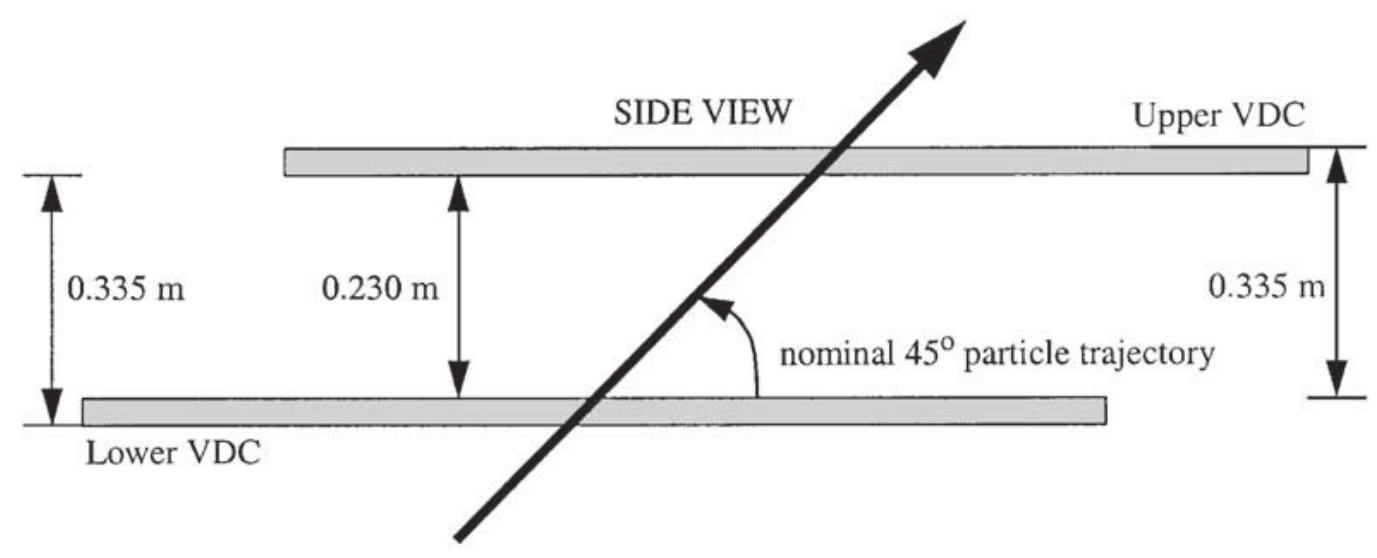

TOP VIEW

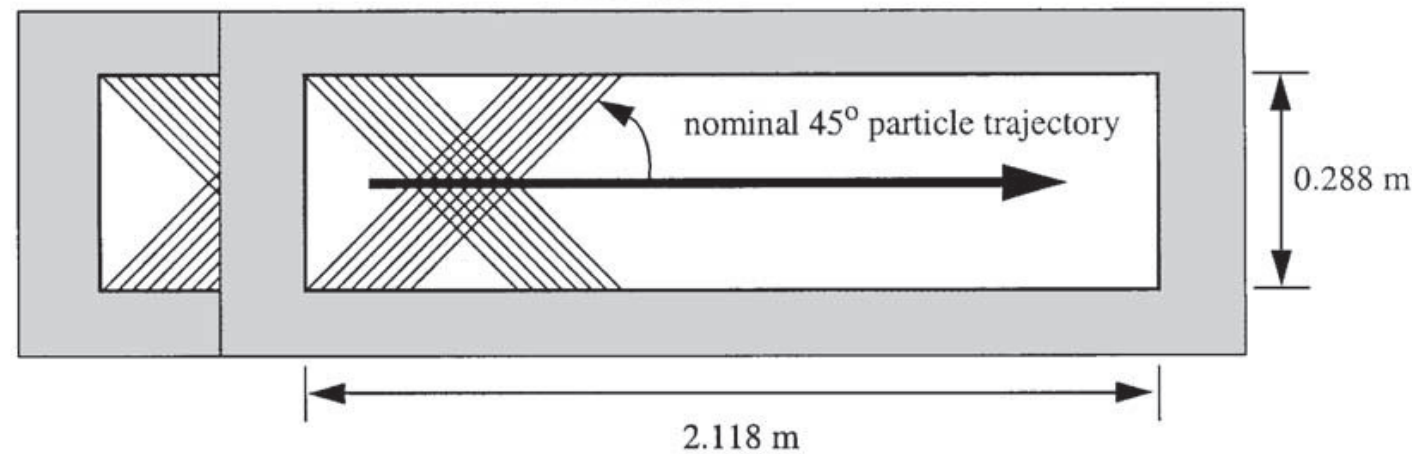

FIG. 3.7: Schematic layout of the VDCs (not to scale).

stream, and complex tracking algorithms are applied offline to provide information about the position and direction of the track.

\section{Scintillators}

The S2m scintillator plane provides triggering and timing information for each HRS. The S2m plane consists of sixteen bars made of fast plastic scintillator (Eljen EJ-230). Each bar measures 17 in by 5.5 in by 2 in thick, and is wrapped with 25 $\mu \mathrm{m}$ of mylar and $50 \mu \mathrm{m}$ of black tedlar. Trapezoidal lucite light guides on both ends couple the bar to 2" photo multiplier tubes (Photonis XP2282B). Fig. 3.8 shows a schematic layout of the S2m plane.

Although the $\mathrm{S} 2 \mathrm{~m}$ plane is relatively thick, it is located behind the tracking 


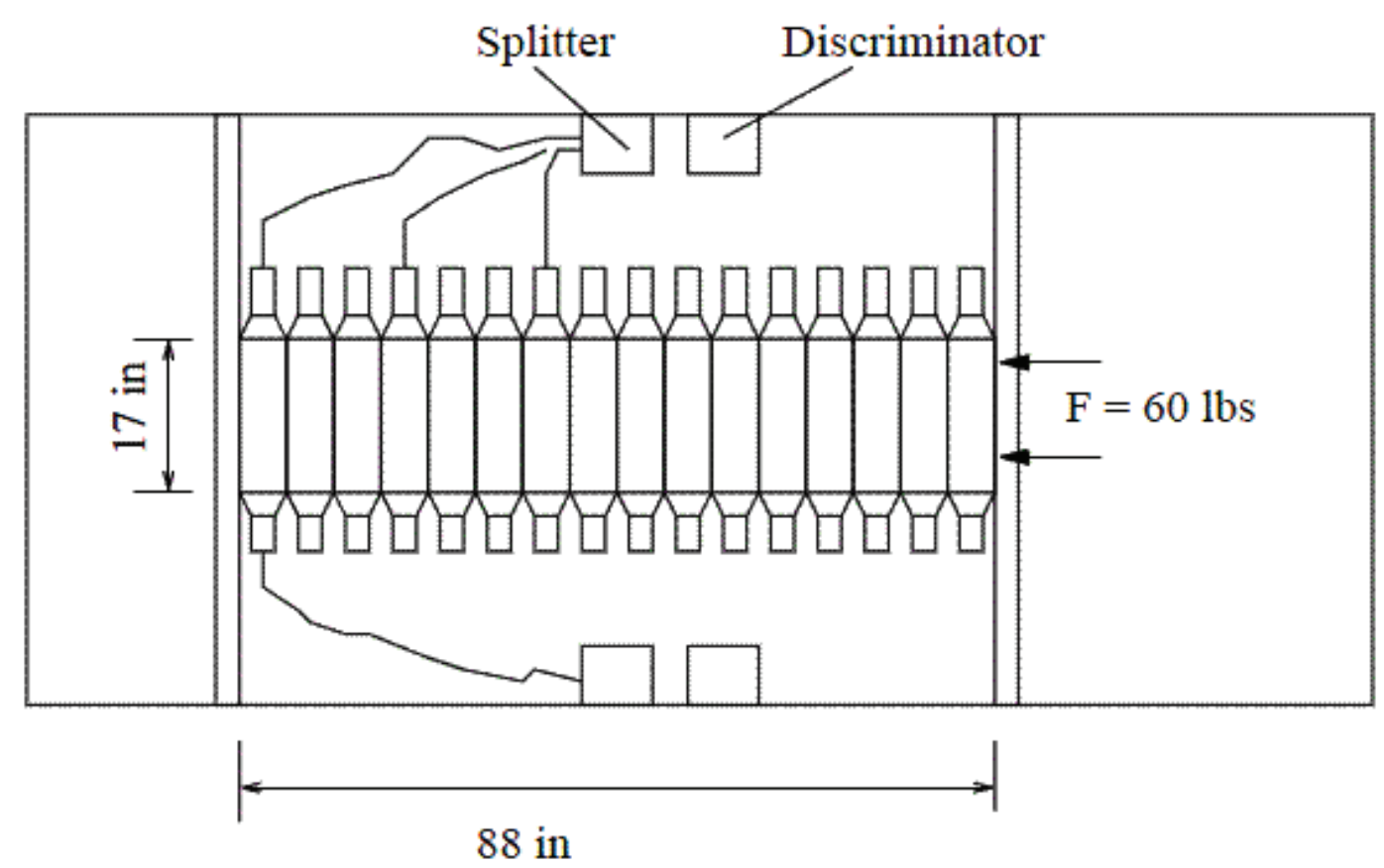

FIG. 3.8: Schematic layout of the S2m scintillator plane.

VDCs and the Gas Cherenkov and does not compromise particle detection. For cosmic rays, each PMT observes about 900 photo-electrons. The average timing resolution for each PMT is $\sigma_{p m t}<150 \mathrm{ps}$ [6].

An additional S0 scintillator paddle is used for the timing calibration of the detectors. The $\mathrm{S} 0$ paddle is a $10 \mathrm{~mm}$ thick BICRON 408 plastic scintillator with an active area that is $170 \mathrm{~cm}$ long by $25 \mathrm{~cm}$ wide. Each end of the paddle is coupled to a 3" PMT (Photonis XP4312B). The S0 paddle is located between the VDCs and the gas Cherenkov detector, and is vertically oriented (perpendicular to the $\mathrm{S} 2 \mathrm{~m}$ paddles). The timing resolution of the $\mathrm{S} 0$ counter is $\sigma_{t} \approx 200 \mathrm{ps}[6]$.

\section{Gas Cherenkov Detectors}

Particle identification is provided by a gas Cherenkov detector filled with $\mathrm{CO}_{2}$ at atmospheric pressure that is mounted between the $\mathrm{S} 0$ counter and the $\mathrm{S} 2 \mathrm{~m}$ plane. The detector consists of ten spherical mirrors with an $80 \mathrm{~cm}$ focal length, each viewed 

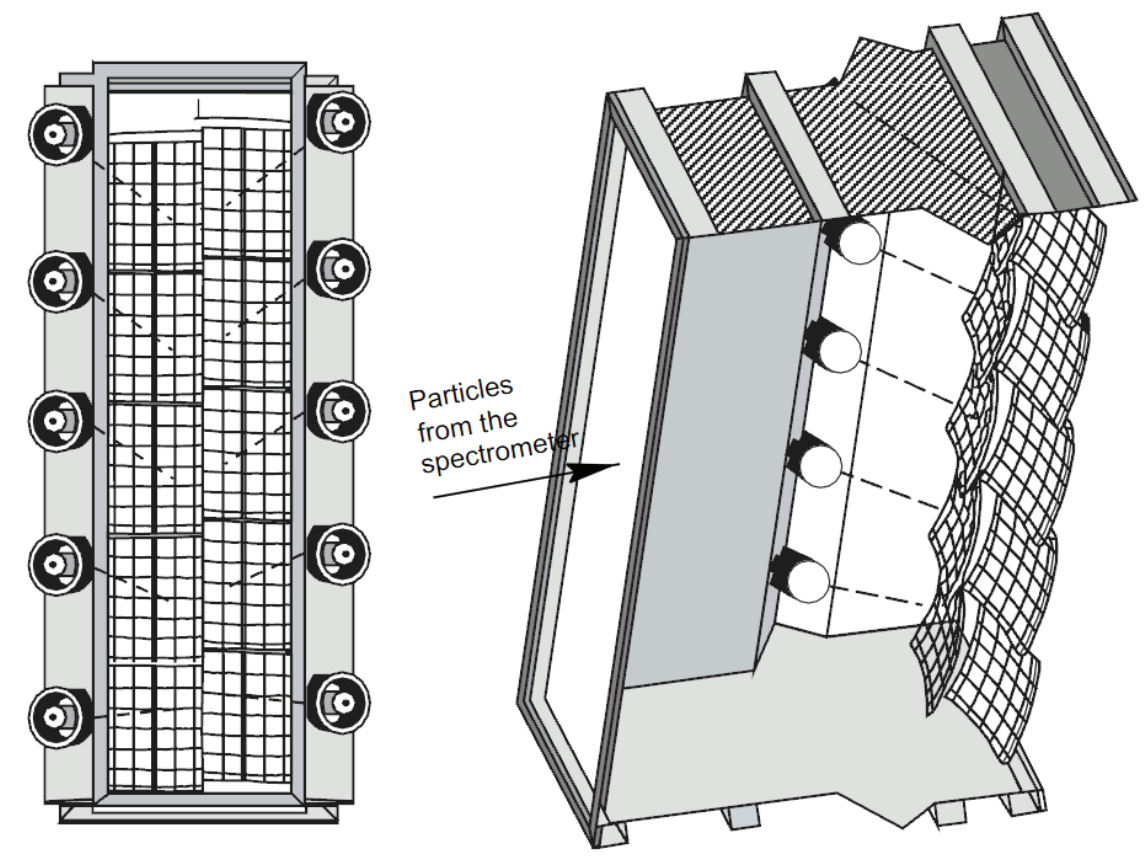

FIG. 3.9: Schematic layout of the gas Cherenkov detector.

by a PMT (Burle 8854). The mirrors are placed at the back of the detector near the output window and are grouped into two columns of five mirrors (Fig. 3.9p.

If a charged particle passes through a dielectric medium at a speed greater than the phase velocity of light in that medium, such that $v / c>1 / n$ (where $\mathrm{n}$ is the index of refraction of the medium), the particle emits Cherenkov light. When Cherenkov light is produced in the gas Cherenkov detector, the light reflects off of the mirrors into the PMTs. For electrons, the momentum threshold to produce Cherenkov light in our detector is $0.017 \mathrm{GeV} / c$, which is sufficiently low. For pions, the momentum threshold is $4.8 \mathrm{GeV} / c$, which is above the momentum acceptance range of the HRSs $(4 \mathrm{GeV} / c)$. Detection of Cherenkov radiation can thus be used to tag electrons and can even be used as part of the online trigger of the HRS. 

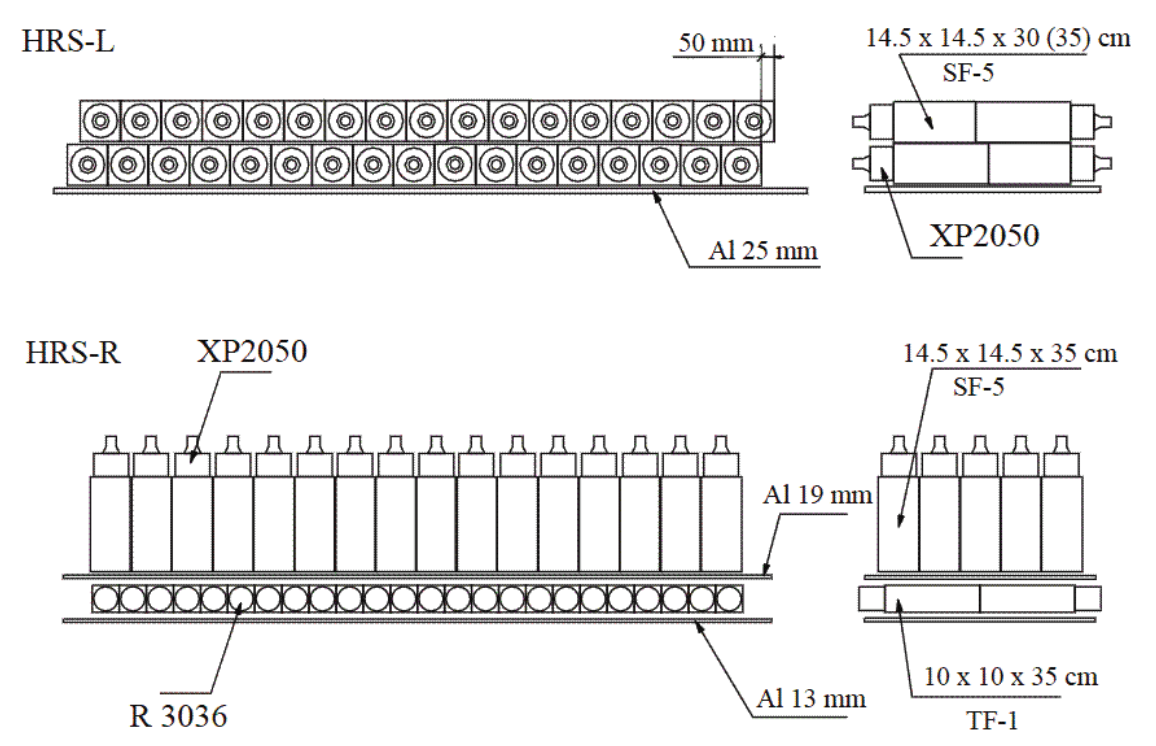

FIG. 3.10: Schematic layout of the shower detectors in the left HRS (top) and right HRS (bottom). Particles enter from the bottom of the figure.

\section{Electromagnetic Calorimeters}

The electromagnetic calorimeters, or shower detectors, also provide good particle identification. The shower detectors of both HRSs are located behind the S2m plane. However, their configurations are slightly different for each spectrometer. Fig. 3.10 shows the structure of the shower detectors for both HRSs. The blocks in both layers in the left HRS (used to detect electrons) and the first layer of the right HRS (used to detect positrons) are oriented perpendicular to the particle tracks. In the second layer of the right HRS, the blocks are parallel to the tracks. Both layers of the left HRS consist of 34 lead glass blocks of dimensions $15 \mathrm{~cm}$ by $15 \mathrm{~cm}$ by $30(35) \mathrm{cm}$. The front layer of the right HRS consists of 48 lead glass blocks of dimensions $10 \mathrm{~cm}$ by $10 \mathrm{~cm}$ x $35 \mathrm{~cm}$, and the second layer consists of 80 lead glass blocks of dimensions $15 \mathrm{~cm}$ by $15 \mathrm{~cm}$ by $35 \mathrm{~cm}$. All blocks are coupled to a single PMT (Photonis XP2050) [6].

The energy of electrons is fully absorbed in the shower detectors. About 20\% of hadrons pass through the shower detectors without interaction, releasing only 
ionization energy. Of the other $80 \%$, many particles escape from the detector with a large fraction of their initial energy. Plotting the ratio of energy deposited in the shower detectors to the particle's initial momentum can thus be used for identification of electrons.

\subsubsection{Data Acquisition}

Experiments in Hall A use the CEBAF On-line Data Acquisition system (CODA) to collect data during the experiment. CODA [7] is the standard data acquisition system designed for use at Jefferson Lab. It provides software tools for monitoring, accumulating, recording, and decoding data taken during experiments.

The raw signals from detectors are first amplified and then sent to Analog-toDigital converters (ADCs), which are used to measure the integrated charge of the raw signals. Copies of the signals are also sent to discriminators, which produce new logical signals with fixed width and amplitude for incoming signals with amplitudes greater than the discriminator threshold. These logical signals are then sent to Time-to-Digital converters (TDCs) and scalers to provide timing and counting information. These modules are all installed on the front-end crates. The operation of modules in a single crate is controlled by the Readout Controller (ROC). ROCs are single-board computers mounted at the beginning of each crate. Each ROC is loaded with a programming script that specifies the types of modules in the crate, their positions within the crate, and certain properties of each module (such as the number of channels). ROCs also manage the communication of the crate through an Ethernet network that transports data from the modules to the CODA Event Builder (EB). The EB is a program that collects information from all modules and constructs a single data structure. This structure is called an event. The data is then sent to the CODA Event Recorder (ER), which records the event. 
The trigger supervisor (TS) decides which events are recorded and which are rejected based on the experiment specific triggering system. Trigger signals are accepted by the TS through eight input channels, T1 to T8. The TS decides which trigger signals to accept based on a set of scaling factors called pre-scale factors, and is capable of accepting multiple triggers. When a trigger signal is accepted, the TS returns a Level One Accept (L1A) pulse, which tells the ROCs to start reading data from the modules. During this data readout, the TS is unable to accept any additional signals until the ROCs are finished processing the data. The time that the TS is unable to record new triggers is called DAQ dead-time. Dead time tells us the percentage of triggers (good events) that were not recorded and can be determined by comparing the number of recorded CODA events with the number of scaler events (see Sec. ??).

In addition to the CODA system, Experimental Physics and Industrial Control System (EPICS) is used to provide slow, real-time information about the incoming beam, target, and spectrometer magnets. This data is typically stored into a raw data file with ASCII format every few seconds.

During the experiment, data taking is controlled by operators in the Hall A counting house using the CODA graphical user interface (GUI) known as Run Control. The Run Control GUI is first used to load configuration scripts to all considered parts of the DAQ for proper readout of the detectors. After CODA is properly configured, the GUI is used to start and stop the data acquisition. During data taking, the GUI allows the user to check the data recording rate and deadtime. The data recorded between each start and stop is called a run. Each run is assigned a sequential run number and is written to a local disk array. Recorded runs are later sent to a tape silo called the Mass Storage System (MSS) for long-term storage. 


\section{CHAPTER 4}

\section{The APEX Experiment}

The APEX experiment will search for an $A^{\prime}$ with a mass ranging from 50 to 550 $\mathrm{MeV}$ and couplings as small as $\alpha^{\prime} / \alpha_{\mathrm{fs}} \approx 10^{-7}[8]$. The experiment will measure the invariant mass spectrum of $e^{+} e^{-}$pairs produced by electron scattering on a high- $Z$ target. This spectrum will be scanned for a narrow peak with a width corresponding to the mass resolution of the experiment. The $e^{-}$and $e^{+}$will be detected in the High Resolution Spectrometers (HRSs). The HRSs contain detectors to accurately measure the momentum, direction, and identity of the incoming particles.

In this chapter I will discuss $A^{\prime}$ production in fixed target experiments and give an overview of the APEX experimental setup.

\subsection{A' Production in Fixed Target Experiments}

An $A^{\prime}$ can be produced by colliding charged particles with nuclei, and can decay into $e^{+} e^{-}$or $\mu^{+} \mu^{-}$pairs. An electron beam scattering off a high-Z target will 


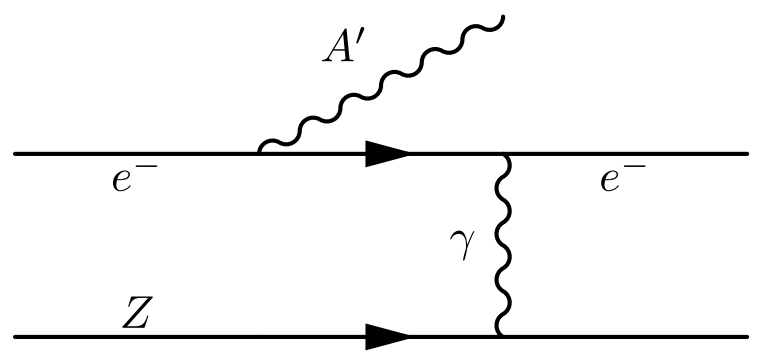

FIG. 4.1: $A^{\prime}$ production by bremsstrahlung from an incoming electron scattering off protons in a target with atomic number $\mathrm{Z}$.

produce $A^{\prime \prime}$ s through bremstrahlung reactions with a cross section

$$
\sigma_{A^{\prime}} \sim 100 \mathrm{pb}\left(\frac{\epsilon}{10^{-4}}\right)^{2}\left(\frac{100 \mathrm{MeV}}{m_{A^{\prime}}}\right)^{2}
$$

where $m_{A^{\prime}}$ is the mass of the $A^{\prime}$ and $\epsilon^{2} \equiv \alpha^{\prime} / \alpha$ is the ratio of the $A^{\prime}$ and electromagnetic fine structure constants [9]. This cross section is several orders of magnitude greater than the $A^{\prime}$ production cross sections in colliding electron and hadron beams [8]. Electron fixed-target experiments also have a high luminosity and favorable kinematics, and are therefore perfectly suited for searching for the $A^{\prime}$ in the sub-GeV mass range.

An $A^{\prime}$ is radiated from electrons scattering off target nuclei in a process analogous to ordinary photon bremsstrahlung (Fig. 4.1). When a charged particle decelerates from the deflection of other charged particles, the moving particle loses kinetic energy in the form of a photon. This process is known as bremsstrahlung.

The production of $A^{\prime \prime}$ s from scattered electrons can be reliably estimated using the Weizsäcker-Williams approximation [10]. For an incoming electron with energy $E_{0}$, the differential cross section to produce an $A^{\prime}$ with mass $m_{A^{\prime}}$ and energy $E_{A^{\prime}} \equiv$ $x E_{0}$ (where $x$ is a constant less than one) is

$$
\frac{d^{2} \sigma}{d x d \cos \left(\theta_{A^{\prime}}\right)} \approx \frac{8 Z^{2} \alpha^{3} \epsilon^{2} E_{0}^{2} x}{U^{2}} \tilde{\chi} \times\left[\left(1-x+\frac{x^{2}}{2}\right)-\frac{x(1-x) m_{A^{\prime}}^{2}\left(E_{0}^{2} x \theta_{A^{\prime}}^{2}\right)}{U^{2}}\right]
$$


where $\mathrm{Z}$ is the atomic number of the target, $\alpha \simeq 1 / 137, \theta_{A^{\prime}}$ is the angle in the lab frame between the emitted $A^{\prime}$ and the incoming electron,

$$
U\left(x, \theta_{A^{\prime}}\right)=E_{0}^{2} x \theta_{A^{\prime}}^{2}+m_{A^{\prime}}^{2} \frac{1-x}{x}+m_{e}^{2} x
$$

is the virtuality of the intermediate electron in initial-state bremsstrahlung, and $\tilde{\chi} \equiv \chi / Z^{2} \sim 0.1-10$ is the reduced Weizsäcker-Williams effective photon flux, which depends on kinematics, atomic screening, and nuclear size effects. The above results are valid for

$$
m_{e^{-}} \ll m_{A^{\prime}} \ll E_{0}, \quad x \theta_{A^{\prime}}^{2} \ll 1 .
$$

Dropping $m_{e^{-}}$and performing the angular integration gives us

$$
\frac{d \sigma}{d x} \approx \frac{8 Z^{2} \alpha^{3} \epsilon^{2} x}{m_{A^{\prime}}^{2}}\left(1+\frac{x^{2}}{3(1-x)}\right) \tilde{\chi}
$$

The rate and kinematics of massless bremsstrahlung and massive $A^{\prime}$-strahlung differ in several important ways 8 :

- The $A^{\prime}$ production rate is proportional to $\frac{\alpha^{3} \epsilon^{2}}{m_{A^{\prime}}^{2}}$. Therefore, it is suppressed relative to photon bremsstrahlung by $\epsilon^{2} \frac{m_{e-}^{2}}{m_{A^{\prime}}^{2}}$. Additional suppression can occur for small $\tilde{\chi}$ when $m_{A^{\prime}}$ is large or $E_{0}$ is small.

- When $x \approx 1$ (so that $E_{A^{\prime}} \approx E_{0}$ ), $U(x, 0)$ is minimized and the production rate is sharply peaked. When the $A^{\prime}$ is produced, it carries nearly all of the beam energy.

- $A^{\prime}$ emission is dominated at angles $\theta_{A^{\prime}}$ such that $U\left(x, \theta_{A^{\prime}}\right) \lesssim 2 U(x, 0)$ (beyond this point, wide-angle emission falls as $\left.1 / \theta_{A^{\prime}}^{4}\right)$. For $x$ near its median value, the 
cutoff emission angle is

$$
\theta_{A^{\prime} \max } \sim \max \left(\frac{\sqrt{m_{A^{\prime}} m_{e^{-}}}}{E_{0}}, \frac{m_{A^{\prime}}^{3 / 2}}{E_{0}^{3 / 2}}\right)
$$

This is much smaller than the opening angle of the $A^{\prime}$ decay products, which is $\sim m_{A^{\prime}} / E_{0}$

Using equation (4.5), we can obtain approximate expressions for the rate of $A^{\prime}$ production. These approximations are correct within about one order of magnitude [8]. The number of $A^{\prime}$ s produced when $N_{e^{-}}$electrons scatter in a target of thickness $T \ll 1$ radiation lengths is

$$
N_{A^{\prime}} \sim N_{e^{-}} \frac{N_{0} X_{0}}{A} T \frac{Z^{2} \alpha^{3} \epsilon^{2}}{m_{A^{\prime}}^{2}} \tilde{\chi} \sim N_{e^{-}} \zeta T \epsilon^{2} \frac{m_{e^{-}}^{2}}{m_{A^{\prime}}^{2}}
$$

where $X_{0}$ is the radiation length of the target in $\mathrm{g} / \mathrm{cm}^{2}, N_{0} \simeq 6 \times 10^{23} \mathrm{~mole}^{-1}$ is Avogadro's number, and $A$ is the target atomic mass in $\mathrm{g} / \mathrm{mol}$. The numerical factor $\zeta \approx 5$ is logarithmically dependent on the choice of nucleus and on $m_{A^{\prime}}$ [10]. For a Coloumb of incident electrons

$$
\frac{N_{A^{\prime}}}{\text { Coulomb }} \sim 10^{6} \tilde{\chi}\left(\frac{T}{0.1}\right)\left(\frac{\epsilon}{10^{-4}}\right)^{2}\left(\frac{100 \mathrm{MeV}}{m_{A^{\prime}}}\right)^{2}
$$

\subsection{Signal and Trident Kinematics}

The $A^{\prime}$ signal will appear as a small resonance in the QED trident invariant mass spectrum. The kinematic differences between the $A^{\prime}$ signal and QED trident backgrounds are of primary consideration in selecting the optimal spectrometer settings for APEX. In this section we will discuss the kinematics of these processes.

The dominant QED backgrounds for $A^{\prime}$ production are the radiative trident 
and Bethe-Heitler trident reactions. The diagrams of these two processes are shown in Fig. 4.2. The production of these trident background events was simulated using the nuclear elastic and inelastic form-factors found in [11]. The simulation was done using MadGraph and MadEvent [12]. MadGraph is a matrix element generator written in the Python programming language, and MadEvent is a package derived from MadGraph used to simulate events. The effect of nuclear excitations on the kinematics in inelastic processes were neglected. The MadEvent code was also modified to account for the nucleus-electron kinematics [8].

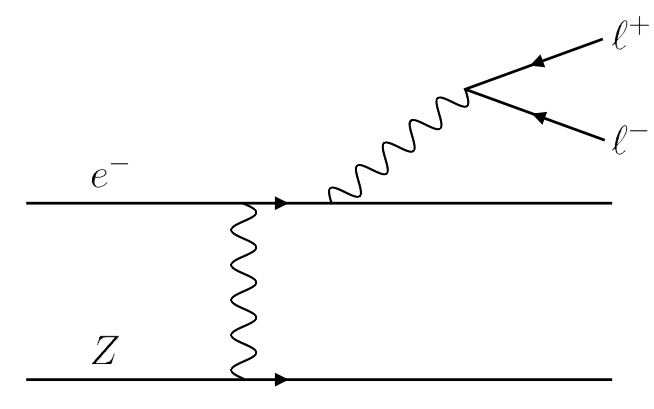

(a)

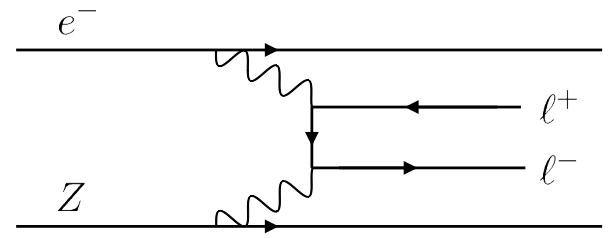

(b)

FIG. 4.2: Diagrams of (A) radiative trident and (B) Bethe-Heitler trident reactions that comprise the QED background of the $A^{\prime} \rightarrow \ell^{+} \ell^{-}$search channels.

$A^{\prime}$ signal events have the same kinematics as radiative trident events whose invariant mass is inside a small window centered at the mass of the $A^{\prime}$. The rate of $A^{\prime}$ events is related to the radiative trident cross section by

$$
\frac{d \sigma\left(e^{-} Z \rightarrow e^{-} Z\left(A^{\prime} \rightarrow \ell^{+} \ell^{-}\right)\right)}{d \sigma\left(e^{-} Z \rightarrow e^{-} Z\left(\gamma * \rightarrow \ell^{+} \ell^{-}\right)\right)}=\left(\frac{3 \pi \epsilon^{2}}{2 N_{\mathrm{eff}} \alpha}\right)\left(\frac{m_{A^{\prime}}}{\delta m}\right)
$$

where $N_{\text {eff }}$ is the number of available decay products and $\delta m$ is the width of the invariant mass window [10]. A Monte Carlo simulation was performed simulating $A^{\prime}$ signal events and radiative background events restricted to a small mass window $\delta m$. The simulation showed almost perfect agreement with equation (4.9) [8]. Therefore, 


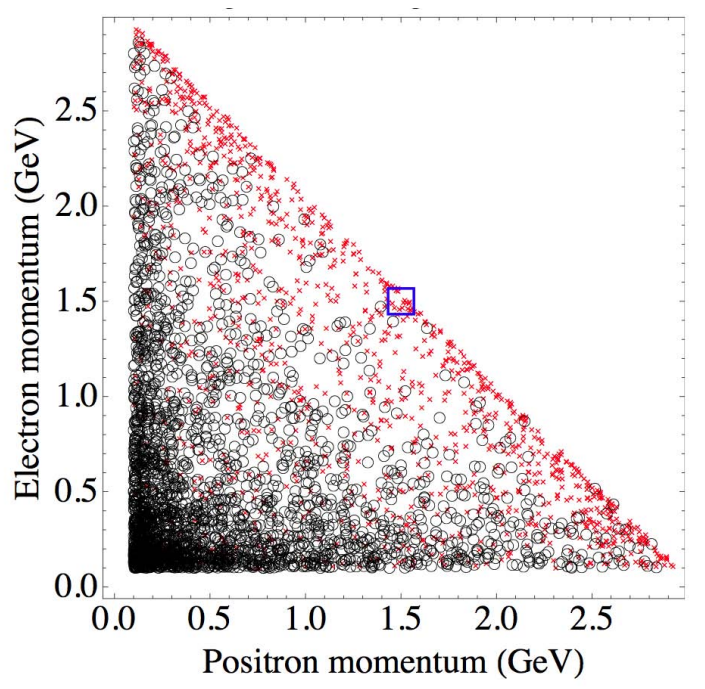

FIG. 4.3: Electron momentum vs. positron momentum for $A^{\prime}$ signal events with $m_{A^{\prime}}=200 \mathrm{MeV}$ (red crosses) and for Bethe-Heitler background events (black circles) using a $3 \mathrm{GeV}$ beam [8]. The blue box shows a spectrometer acceptance window that optimizes signal sensitivity.

a radiative background subsample can be used to analyze the $A^{\prime}$ signal.

The Bethe-Heitler process has a much larger cross section than both the signal and radiative processes, but much different kinematics. $A^{\prime}$ s are produced forward carrying most of the beam energy, while the recoiling electrons are soft and scatter at wide angles. In contrast, the Bethe-Heitler processes are not enhanced at high pair energies. They also possess a forward singularity that strongly favors an asymmetric configuration with one energetic, forward electron or positron and the other constituent of the pair much softer [10]. Fig. 4.3 shows the electron vs. positron momenta for the $A^{\prime}$ signal events (red crosses) and Bethe-Heitler background events (black circles). The signal $e^{+} e^{-}$pairs are concentrated in the region where $E\left(e^{+}\right)+E\left(e^{-}\right) \approx E_{\text {beam. }}$. Bethe-Heitler background rejection is optimized when the two spectrometers have equal angles and momentum acceptances equal to half the beam energy. This acceptance window is shown by the blue box in Fig. 4.3 . 


\section{Calculation of the $\epsilon$ reach}

To set a limit on the coupling $\alpha^{\prime}$ we use a ratio method that normalizes $A^{\prime}$ production to measured QED trident background rate. This method will set a limit on $\epsilon^{2}$, or $\alpha^{\prime} / \alpha_{\mathrm{fs}}$, while minimizing systematic uncertainty from acceptance and trigger efficiencies [13]. $A^{\prime}$ production is simply related to radiative production through Eq. 4.9. The ratio $f$ of the radiative-only cross section to the full trident cross section can be reliably computed using MadGraph and MadEvent. The results of the simulations show that $f$ varies linearly from 0.21 to 0.25 across the APEX mass range with an uncertainty of 0.01 . This uncertainty dominates over the Monte Carlo statistical uncertainty and possible next-to-leading order QED effects [13]. By scaling the radiative cross section in Eq. 4.9 by $f$ we compute the upper limit on $\alpha^{\prime} / \alpha_{\mathrm{fs}}$ using

To calculate the $\alpha^{\prime} / \alpha_{\mathrm{fs}}$ reach of the proposed experiment we first calculate the rates of all reactions entering into the spectrometer acceptance by integrating over the target profile used in each kinematic setting (see Table 4.1). Trident rates were calculated as a function of invariant mass. An estimate of the mass resolution $\delta m$ obtainable for APEX is calculated, and the bin size of the invariant mass acceptance region is set to $2.5 \times \delta m$. The total number of signal events $S$ is calculated using Eq. 4.7, and the number of background events is calculated using $S / \sqrt{B}=2$. The resulting limit for the full experiment is shown in Fig. 4.4.

\subsection{Accidentals and rates}

The final APEX production data will be comprised of coincidences between the two spectrometers, many of which are accidental coincidence events. A coinci- 


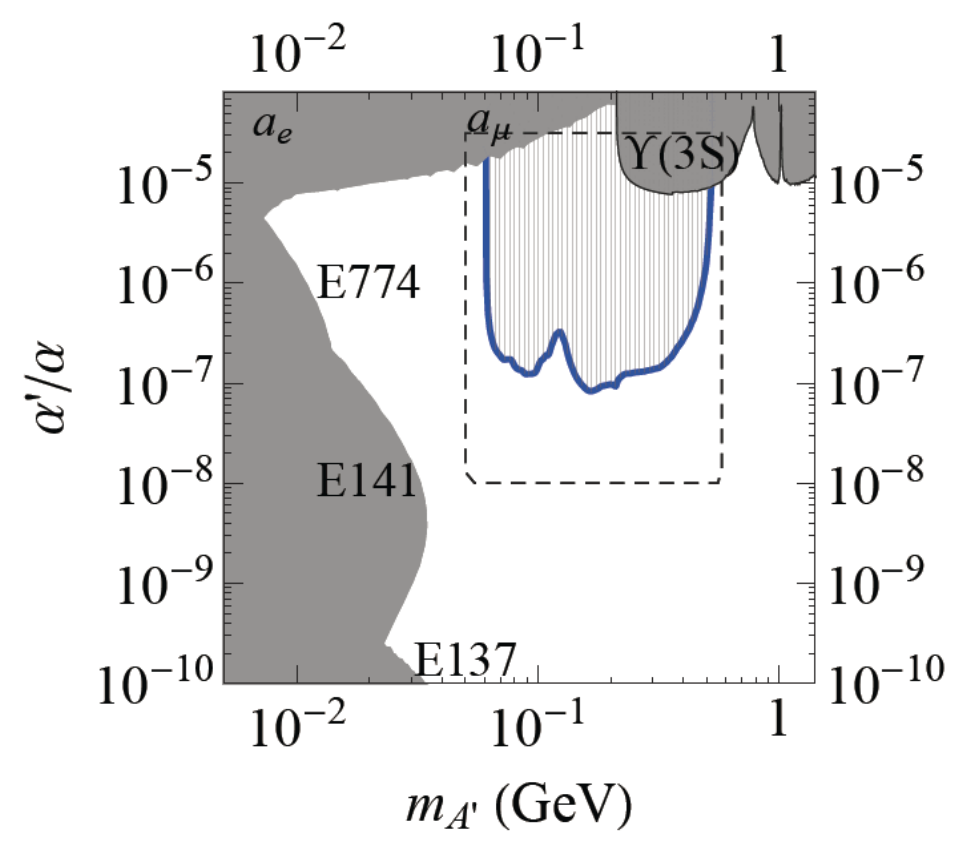

FIG. 4.4: Anticipated $2 \sigma$ sensitivity for APEX, with existing constraints on an $A^{\prime}$ from electron and muon anomalous magnetic moment measurements, $a_{e}$ and $a_{\mu}$ (see [14]), the BaBar search for $\Upsilon(3 S) \rightarrow \gamma \mu^{+} \mu^{-}$[15], and three beam dump experiments, E137, E141, and E774 [16, 17, 18] (see [19]).

dence trigger is produced when both spectrometers detect a significant event within the defined coincidence timing window. A true coincidence occurs when an $e^{+} e^{-}$ pair is produced and both constituents are detected in coincidence. An accidental coincidence occurs when the two particles that produce the coincidence trigger do not come from an $e^{+} e^{-}$pair. The accidental coincidences will mainly consist of accidental $e^{+} e^{-}$and true $e^{-} \pi^{+}$events.

The rate of accidentals can be estimated from the singles rate in each HRS and the duration of the timing window:

$$
N_{\text {accidentals }} \simeq \sigma N_{\text {Left }} N_{\text {Right }}
$$

where $N_{\text {accidentals }}$ is the rate of accidentals, $N_{\text {Left-HRS }}$ and $N_{\text {Right-HRS }}$ are the singles rates of each HRS, and $\sigma$ is the size of the timing window. This means that $N_{\text {accidentals }}$ 
scales with the square of the total coincidence trigger rate.

At small angles, the main contributions to the counting rate in the spectrometers are due to electrons, pions, and protons scattering into the HRS acceptance. Table 4.1 summarizes the expected singles rates for each proposed kinematic setting. Kinematic settings A, B, and D will use a target that provides uniform coverage in scattering angles ranging from $4.5^{\circ}$ to $5.5^{\circ}$. Kinematic setting $\mathrm{C}$ will have the target material concentrated at the ends of the angular acceptance so that the effective angles are $4.5^{\circ}$ and $5.5^{\circ}$, with three times more material at the downstream end $\left(4.5^{\circ}\right)$ than at the upstream end $\left(5.5^{\circ}\right)$.

The table also includes the projected trigger rates. These rates assume a $20 \mathrm{~ns}$ coincidence timing gate and an online $\pi^{+}$rejection of 30 by including the Right-HRS gas Cherenkov counter in the trigger. The observed trigger rates will have contributions from trident processes, the "two-step" trident process, and accidentals. The "two-step" trident process occurs when an electron radiates a real, hard photon in the target that converts to a high-mass $e^{+} e^{-}$pair. For thin targets it is suppressed compared to the trident rates, so it is sub-dominant for all kinematic settings. The accidental coincidence rates are dominated by $e^{+} e^{-}$accidentals, but $\pi^{ \pm}$contributions are also included. The calculation of the accidental rates assumes that $\pi^{+}$and $\left(\pi^{-}\right)$will be rejected offline by a factor of 100 and 3 respectively. It also assumes a $2 \mathrm{~ns}$ wide cut on the coincidence timing spectrum and an additional factor of four rejection from correcting on the target vertex. These rejection factors are quite conservative, and further rejection can be expected by exploiting kinematics (e.g. $\left.E_{e^{+}}+E_{e^{-}} \leq E_{\text {beam }}\right)$.

The rates given in the table have been checked against measurements made by experiment E03-012 [8]. This experiment used a $5 \mathrm{GeV}$ electron beam incident on a hydrogen target with the HRS positioned $6^{\circ}$ relative the beam and an HRS momentum setting of $2 \mathrm{GeV}$. The rates were also checked with the APEX test run 
TABLE 4.1: Singles rates, trigger rates, and coincidence rates (including both true coincidences and accidentals that can not be rejected offline).

\begin{tabular}{|c|c|c|c|c|}
\hline Settings & A & B & $\mathrm{C}$ & $\mathrm{D}$ \\
\hline Beam energy $(\mathrm{GeV})$ & 2.2 & 4.4 & 1.1 & 3.3 \\
\hline Beam current $(\mu A)$ & 70 & 60 & 50 & 80 \\
\hline Effective angles & $4.5-5.5$ & $4.5-5.5$ & $4.5-5.5$ & $4.5-5.5$ \\
\hline Target $T / X_{0}\left(\right.$ ratio $\left.{ }^{a}\right)$ & $4 \%$ & $8 \%$ & $0.7 \%(1: 3)$ & $8 \%$ \\
\hline Central momentum of the spectrometers $(\mathrm{GeV})$ & 1.095 & 2.189 & 0.545 & 1.634 \\
\hline Singles (negative polarity) & & & & \\
\hline$e^{-}(\mathrm{MHz})$ & 4.1 & 0.7 & 4.5 & 2.2 \\
\hline$\pi^{-}(\mathrm{MHz})$ & 0.1 & 1.7 & 0.025 & 0.9 \\
\hline Singles (positive polarity) & & & & \\
\hline$e^{+}(\mathrm{kHz})$ & 27 & 5 & 18 & 17 \\
\hline$\pi^{+}[p](\mathrm{kHz})$ & 90 & 1700 & 25 & 900 \\
\hline Trigger $^{b} / \mathrm{DAQ}(\mathrm{kHz})$ & 3.0 & 3.1 & 2.0 & 3.3 \\
\hline Coincidence backgrounds: & & & & \\
\hline Tridents: $e^{-} Z \rightarrow e^{-} e^{+} e^{-} Z(H z)$ & 500 & 110 & 260 & 370 \\
\hline "Two-step" tridents (Hz) & 30 & 16 & 3 & 45 \\
\hline Accidentals ${ }^{c}(\mathrm{~Hz})$ & 55 & 30 & 40 & 40 \\
\hline
\end{tabular}

${ }^{a}$ The $\mathrm{A}, \mathrm{B}$, and D settings all use targets that provide uniform coverage in effective scattering angles from $4.5^{\circ}$ to $5.5^{\circ}$. Setting $\mathrm{C}$ uses a target that is concentrated at the edges of the angular acceptance, so that the effective angles are $4.5^{\circ}$ and $5.5^{\circ}$, with three times more target material at the downstream end $\left(.5^{\circ}\right)$ than the upstream end $\left(4.5^{\circ}\right)$.

${ }^{b}$ The trigger rate assumes a $20 \mathrm{~ns}$ time window and a $\pi^{+}$rejection factor of 30 by including the Right-HRS gas Cherenkov counter in the trigger.

${ }^{c}$ Assumes offline $\pi^{+}$rejection by a factor of $100, \pi^{-}$rejection by a factor of 3 , a 2 ns true coincidence time window, and an additional factor of four rejection from correcting on the target vertex.

data (see Sec. 7.7).

\subsection{Experimental Setup}

High-intensity fixed-target beams and high precision spectrometers are ideally suited to search for a new $A^{\prime}$ particle. The $A^{\prime}$ production rate, the luminosity, and the mass resolution attainable at Jefferson Lab vastly exceed what is available using colliding electron beam facilities. The APEX Experiment will use Jefferson Lab's Continuous Electron Beam Accelerator Facility (CEBAF) and the High Resolution 


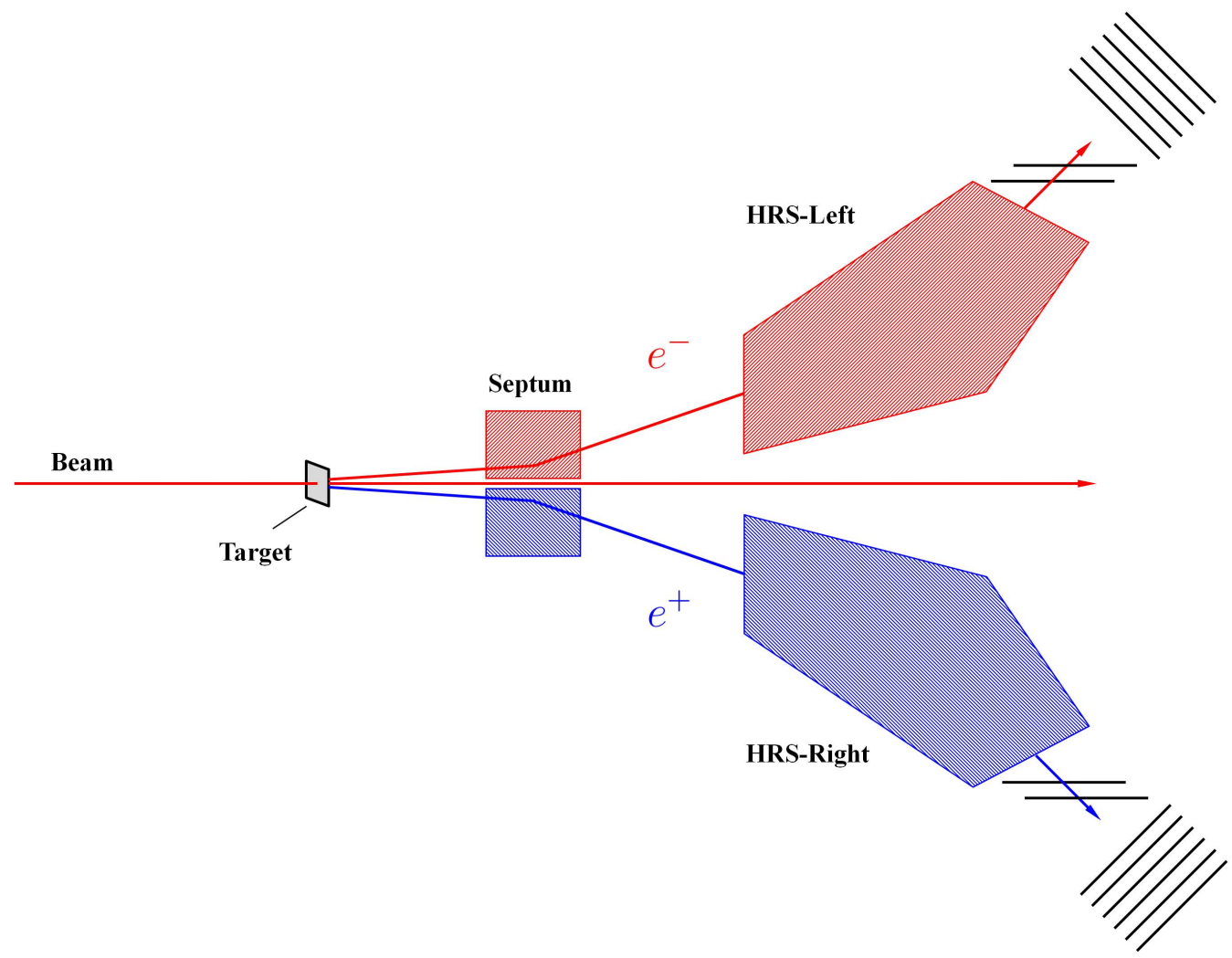

FIG. 4.5: The layout of the experimental setup. The experiment will study $e^{+} e^{-}$ pairs produced off an electron beam incident on a high- $Z$ target. The septum magnets allow detection of $e^{-}$and $e^{+}$at angles of $5^{\circ}$ relative to the incident beam. The HRSs will be used to detect the $e^{-}$and $e^{+}$.

Spectrometers (HRS) in Hall A to search for the $A^{\prime}$. The proposed experiment will probe couplings $\alpha^{\prime} / \alpha \gtrsim 10^{-7}$ and masses $m_{A^{\prime}} \sim 50-550 \mathrm{MeV}$.

The experiment will study $e^{+} e^{-}$production off an electron beam incident on a tungsten target as illustrated in Fig. 4.5. The two HRSs will be used to detect the $e^{+} e^{-}$pairs and measure their invariant mass. Electrons will be detected in the LeftHRS and positrons will be detected in the Right-HRS. The invariant mass spectrum will be scanned for a narrow peak with a width corresponding to the instrumental resolution. The relative mass resolution will be $0.5 \%$, limited by multiple scattering in the target material, track measurement errors by the HRS detectors, and imperfections in the magnetic optics reconstruction matrix (see Sec. 7.6). 
Dipole septum magnets between the target and the HRS aperture will allow the detection of $e^{-}$and $e^{+}$at angles of $5^{\circ}$ relative to the incident beam. An elongated target with ten tungsten ribbons spaced along the beam line will increase the mass coverage by providing a variation of $\pm 0.5^{\circ}$ in the scattering angle. The $e^{+} e^{-}$pairs will be detected in coincidence within a timing window of 20 ns. The rejection of pion backgrounds will be done online using the Right-HRS gas Cherenkov detector.

\subsubsection{Target}

There are several factors that need to be considered when designing the target to be used for production data taking. We want to maximize the production rate of $e^{+} e^{-}$pairs while also attaining the best mass resolution possible. To achieve this, we need to minimize multiple scattering of $e^{-}$and $e^{+}$in the target and also have enough material to get a sufficient production rate. The target must be made of a high $\mathrm{Z}$ material in order to maximize the bremsstrahlung to pion production ratio. The stability of the target material at high temperatures also needs to be considered in the design. Finally, using a target elongated along the beamline would provide a wide and uniform mass coverage [8].

The final design of the target contains three sections: a production section (bottom) used for production data taking, a beam-target alignment section (middle) used for aligning the target components with the beam, and an optics section (top) used to perform the optics calibration along the entire length of the target. A drawing of the target is shown in Fig. 4.6.

The production section of the target consists of 10 ribbons of tungsten, each with a thickness of $15 \mu \mathrm{m}$ (0.43\% radiation lengths). The ribbons are $2.5 \mathrm{~mm}$ wide and spaced $5.5 \mathrm{~cm}$ apart along the beamline. The $e^{+} e^{-}$pairs produced within the acceptance of the spectrometers miss all downstream material, so only one ribbon 


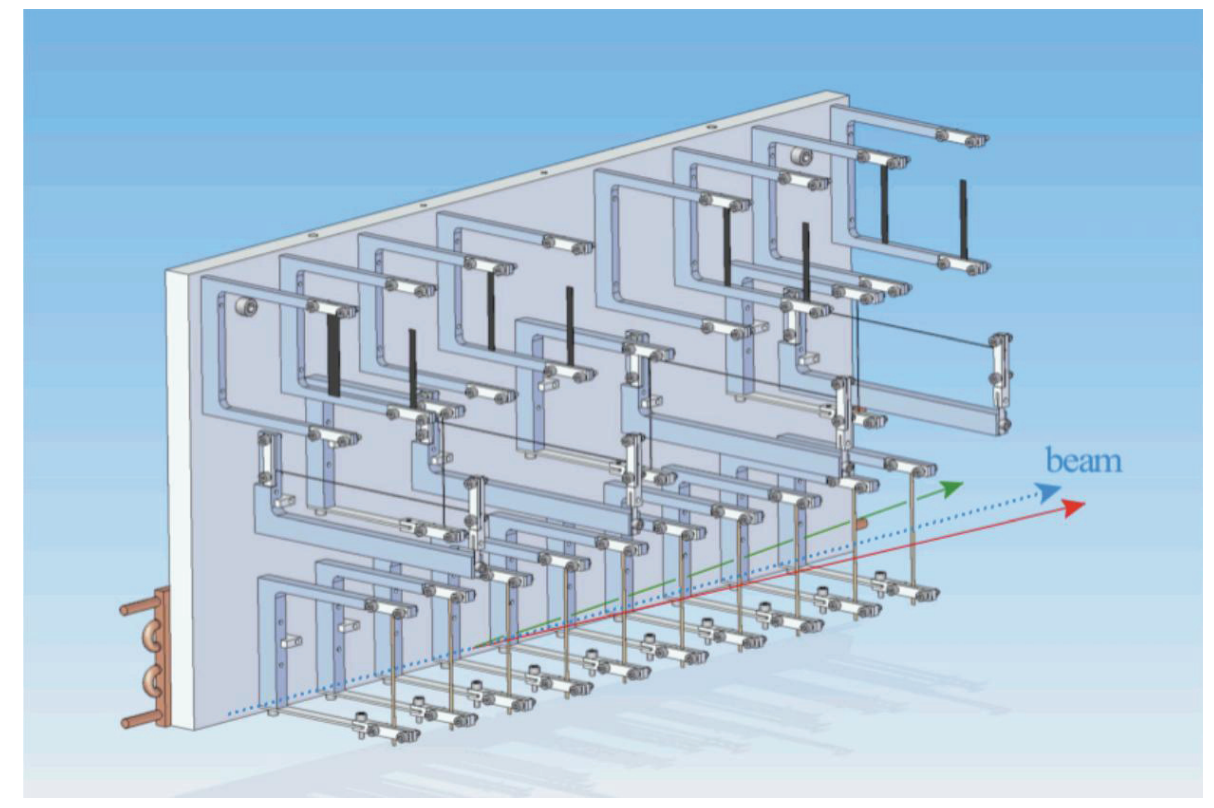

FIG. 4.6: Final design of the APEX target. The target is split into three sections: production section at the bottom, beam-target alignment section in the middle, and optics section at the top.

contributes to multiple scattering in the target. Using 10 targets separated along the beam line provides a variation of $1^{\circ}$ in the scattering angle, which increases the $m_{A^{\prime}}$ coverage by $50 \%$ [20].

The alignment section (middle) consists of 4 horizontal and 3 vertical tungsten, each $100 \mu \mathrm{m}$ in diameter. The horizontal wires are arranged in $5 \mathrm{~mm}$ vertical steps and are located along the beam line at $-25,-10,10$, and $25 \mathrm{~cm}$. The vertical wires are arranged in $2.5 \mathrm{~mm}$ horizontal steps and are located along the beam line at -20 , 0 , and $20 \mathrm{~cm}$. The alignment target system has been surveyed relative to the full target system, so the beam direction relative to the alignment section will provide alignment calibration for the entire APEX target system.

The optics calibration section (top) consists of 8 carbon foils providing calibration points along the full length of the target system. The foils are arranged so that the beam can pass through either 4 or 8 foils at a time in order to provide an initial calibration with either $14 \mathrm{~cm}$ spacing along the beam line or $7 \mathrm{~cm}$ spacing. 
For an electron beam of $80 \mu \mathrm{A}$ incident on a $10 \% X_{0}$ tungsten target, the maximum heat load is about $140 \mathrm{~W}$. The APEX target system includes 4 Lytron CP15 cold plates installed on the back of the primary aluminum mounting plate. All target holders are made of aluminum so that heat is efficiently conducted into the aluminum mounting plate (over $1 \mathrm{~cm}$ thick). By supplying the cooling plates with nitrogen gas, a heat removal of up to $200 \mathrm{~W}$ is readily achievable [8].

\subsubsection{Septum Magnet}

The radiated $A^{\prime}$ 's travel forward at very small angles. The opening angle of the $A^{\prime}$ s decay products is also small. The two High Resolution Spectrometers used to detect the decay products can pivot freely around the center of Hall A; however, they can only rotate to a minimum angle of $12.5^{\circ}$ with respect to the beamline. In order to detect $e^{+} e^{-}$pairs at small angles, room temperature dipole septum magnets will be installed between the target and the spectrometers. The septum magnets will bend particles scattered at angles as small as $5^{\circ}$ relative to the incident beam so that their trajectories overlap the trajectories detectable by the HRSs. Sec. 5.2.2 describes how the septum magnets were used during the test run.

\subsubsection{Track Measurement}

Good particle tracking is essential for precise reconstruction of a particle's momentum vector at the target. Two vertical drift chambers (VDCs) in each spectrometer are used for the measurement of the track's coordinates and direction in the focal plane (see Sec. 3.2.3). The VDCs have a spatial resolution $\sigma_{x, y} \approx 100 \mu \mathrm{m}$ and an angular resolution $\sigma_{\theta, \phi} \approx 0.5 \mathrm{mrad}$.

APEX will experience track rates of up to $5 \mathrm{MHz}$. Operation of the HRS tracking at a rate higher than $100 \mathrm{kHz}$ is uncommon. However, by modifying the 
detector electronics, using the VDC at a maximum track rate of $5-6 \mathrm{MHz}$ can be justified. A track rate of $30-50 \mathrm{kHz}$ of elastic scattering events from a ${ }^{12} \mathrm{C}$ target was used during many HRS optics calibrations. This rate of elastic events corresponds to a track density of $\sim 10 \mathrm{kHz}$ per $\mathrm{cm}$ of the chamber length [8]. The total length of the chamber is $2 \mathrm{~m}$, so operation of the HRS tracking should be possible at a rate of $2 \mathrm{MHz}$ for the whole chamber under standard operational voltage.

The VDC read-out system uses amplifier/discriminator (A/D) cards to read out the wire signals. Previously, LeCroy 2475 type cards were used in the VDCs. These cards require a minimum threshold of $5 \mu \mathrm{A}$, which requires a working high voltage of $-4 \mathrm{kV}$ for the VDCs. This limits the maximum rate for stable operation of the HRS tracking. One way to improve the high rate capability of the VDCs is to reduce the gain and increase the corresponding sensitivity of the A/D cards. New A/D cards were developed by JLab's Electronics Group to increase the signal sensitivity by a factor of five [21]. This directly translates to an additional factor of five in rate capability, which brings the VDC rate limit to $10 \mathrm{MHz}$ for the whole chamber.

The VDC has a maximum drift time of $350 \mathrm{~ns}$, which corresponds to 1.75 accidental tracks per event at $5 \mathrm{MHz}$. However, the timing of such accidental tracks will not match the timing of the trigger. Tracks of real coincidence events will reside within a 20 ns timing window, and tracks outside this window will be rejected. Applying this timing condition will reduce the number of accidental tracks by a factor of 10 , or 0.2 accidentals per event at $5 \mathrm{MHz}$. Because this reduction is independent for each VDC, the probability of an accidental track being reconstructed in both VDCs will be at most $5 \%$. In these remaining $5 \%$ of events, the real track will be determined using the fact that its trajectory intersects the proper scintillator paddle of the 16 paddle $\mathrm{S} 2 \mathrm{~m}$ trigger plane (see Sec. 5.3 ). As a result, the probability of a false track drops below $0.5 \%$ [ 8 . 


\subsubsection{SciFi Detector}

The sensitivity of our $A^{\prime}$ search depends critically on precise reconstruction of the invariant mass of $e^{+} e^{-}$pairs. The HRS has an excellent relative momentum resolution of $\mathcal{O}\left(10^{-4}\right)$, so the mass resolution is dominated by the angular resolution of the spectrometers.

The track reconstruction of the spectrometers is normally calibrated using the sieve slit method. The sieve slit is a $5 \mathrm{~mm}$ tungsten plate with a grid of holes drilled through it. It is positioned between the target and the entrance of the spectrometer during optics calibration runs. Electrons scatter off a target and travel toward the sieve slit. The tungsten plates are thick enough so that electrons incident on the plate never enter the acceptance of the spectrometer. Thus, only electrons whose trajectories pass through the holes of the sieve slit enter into the spectrometer. Their tracks are reconstructed back to the sieve plane and their positions at the sieve plane are compared with the carefully surveyed hole locations. By analyzing the deviations between the reconstructed and surveyed hole positions, one can optimize the coefficients used to reconstruct the particle trajectories (see Sec. 6.3).

The sieve slit method works well only when the HRS has a negative polarity. To perform the optics calibration with positive polarity, positrons are produced via pair production off the electron beam incident on the target. Positrons with momentum equal to that of the HRS momentum setting travel toward the sieve plate located at the entrance of the spectrometer. Positrons incident on the sieve plate never enter the HRS acceptance, so only positrons traveling through the sieve holes enter into

the spectrometer. However, there is a high rate of electrons scattering from the target that are also incident on the sieve plate. These electrons then pair produce in the sieve plate and create many positrons with momentum equal to that of the HRS momentum setting. The result is a flood of positrons entering into the spectrometer, 
making the sieve holes impossible to distinguish.

A scintillating fiber detector ( $\mathrm{SciFi}$ ) was designed to allow optics calibration of the HRS with both negative and positive polarity. The SciFi detector will also provide a more thorough calibration over the full spectrometer acceptance. A drawing of the detector is shown in Fig. 4.7. It will be located in the target chamber approximately one meter downstream of the target and immediately upstream of the septum magnet.

The active area of the detector is $8.8 \mathrm{~cm} \times 10.3 \mathrm{~cm}$ and consists of two orthogonal planes of 32 scintillating fibers with a $1 \mathrm{~mm}$ diameter. The active area is mounted to a mechanical arm coupled with a stepper motor, allowing the detector to be precisely positioned inside of the vacuum chamber. The scintillator fibers are optically coupled to clear $1.5 \mathrm{~mm}$ diameter optical fibers that transport the scintillation light to a Hamamatsu 64 channel multi-anode photomultiplier tube (maPMT). The optical fibers are necessary to locate the PMT assembly away from the intense magnetic field and radiation surrounding the beamline. The PMT assembly is a Faraday cage containing the maPMT and four 16 channel amplifier/discriminator cards.

Two SciFi detectors (one for each spectrometer) are being built for APEX. One detector has been fully assembled and is currently being tested. Preliminary results demonstrate a $\sim 7$ photoelectron yield per fiber. Both detectors will be completely assembled and commissioned well before the start of the experiment.

\subsubsection{Particle Identification}

The HRSs are able to detect various types of particles, but APEX is only interested in detecting electrons and positrons. The HRS spectrometer detector packages are equipped with gas Cherenkov counters and two-layer lead-glass calorimeters for 


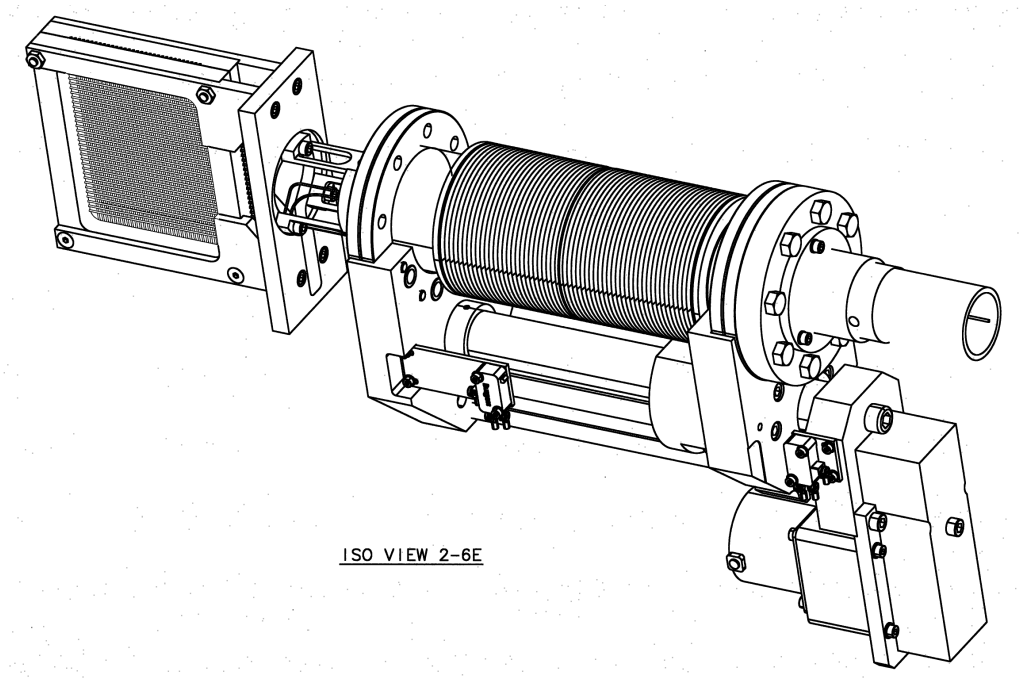

FIG. 4.7: Drawing of the scintillating fiber (SciFi) detector. The SciFi detector will be used for optics calibration of the spectrometers during APEX.

identification of electrons and positrons (see Sec. 3.2.3). Under the APEX configuration settings, the highest projected ratio of rates for $e^{+} / \pi^{+}$is $\sim 1 / 340$ (see Sec. 4.1). Therefore, the production data sample is especially susceptable to $\pi^{+}$contamination. The gas Cherenkov counter of the Right-HRS will be used as part of the coincidence trigger to provide online $\pi^{+}$rejection. This will provide a $\pi^{+}$rejection factor of $\sim 100$ for true coincidence events. The combined pion rejection factor using both the gas Cherenkov counter and the calorimeter in the offline analysis is at least as high as 10,000 [8].

\subsubsection{Trigger and DAQ}

\section{Trigger}

Triggers are signals responsible for prompting the DAQ system to start reading out detector information. A trigger signal can be produced when a particle hits 


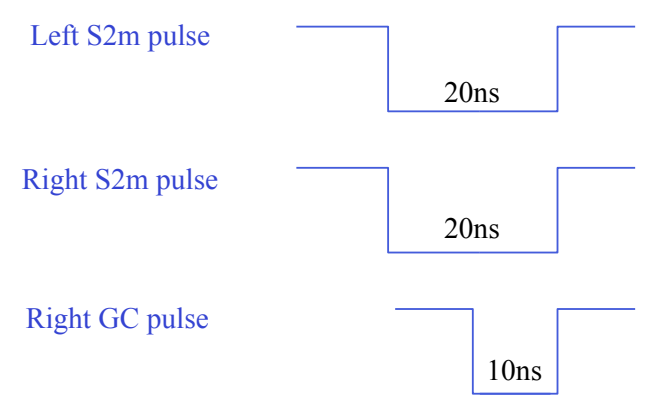

FIG. 4.8: Main coincidence trigger. When pulses from the left S2m plane (20 ns), right S2m plane (20 ns), and right gas Cherenkov (10 ns) overlap, a trigger signal is formed. The overlap of these three pulses creates a $40 \mathrm{~ns}$ coincidence timing window.

a detector or a combination of detectors. The trigger configuration is set up so that detector information is read out only for events of interest. After triggers are created, they are sent to the Trigger Supervisor (TS). The TS decides which trigger to accept according to user-defined pre-scale factors (see Sec. 3.2.4).

The APEX experiment is interested in detecting $e^{-}$and $e^{+}$coincidence events. To select these events, the main trigger will consist of a coincidence between the S2m scintillator planes of both HRSs, and the gas Cherenkov counter of the Right-HRS. The gas Cherenkov counter is used to provide online pion rejection. A 20 ns signal is formed when there is a hit in one of the sixteen paddles of either S2m plane. A 10 ns signal is formed when the Right-HRS gas Cherenkov counter sees a valid signal. When these three signals overlap (Fig. 4.8), the main trigger is formed and detector information is read out to the DAQ system. The triple coincidence of these three signals forms a $40 \mathrm{~ns}$ coincidence timing window.

\section{Sparsification}

During the experiment, the spectrometers will experience high track rates up to $5 \mathrm{MHz}$. As a result, the VDCs will read out a large excess of background hits, 
Common Stop Without Sparsification

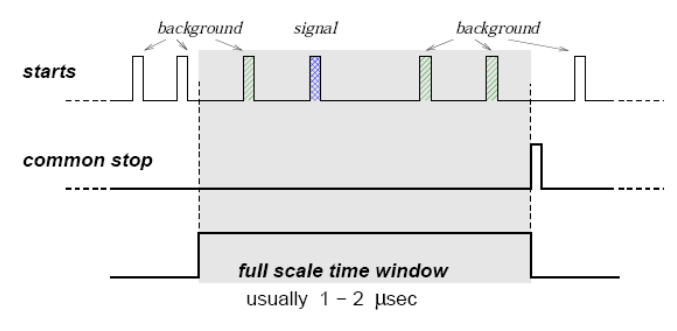

Common Stop

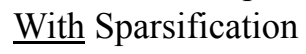

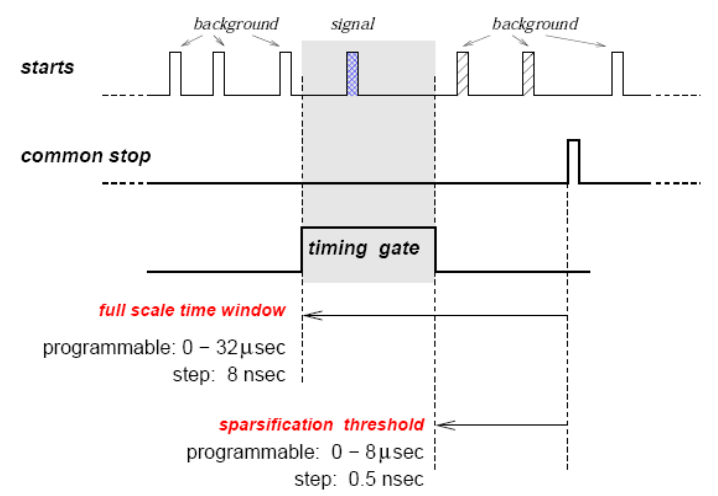

FIG. 4.9: A timing diagram for the 1877S TDC running in common stop mode [22]. Without sparsification, background signals near the stop pulse are recorded. When operating in sparsification mode, these background signals can be ignored.

leading to a significant increase in DAQ dead time.

The VDC signals travel from amplifier discriminator cards to LeCroy 1877S TDCs. These TDCs will operate in common stop mode. When operating in common stop mode, the TDC sees a stop pulse from the Level-1 Accept trigger signal and records all start pulses that occurred within a programmable full scale time window (see Fig. 4.9). There is a considerable delay between the start and stop pulses, so the full scale time window needs to be long enough to record the signals of interest. As a result, many background hits that occur close to the stop pulse are also recorded.

Fortunately, the 1877S TDCs can run in sparsification mode. In sparsification mode, start pulses only get recorded if they occur after the edge of the full scale time window and before the programmed sparsification threshold (see Fig. 4.9). Background pulses that occur close to the stop pulse are thus disregarded. 


\subsection{Proposed Measurement}

The APEX experiment will run during the $12 \mathrm{GeV}$ running period. The experiment will use a beam energy of $1.1 \mathrm{GeV}$ for 6 days, $2.2 \mathrm{GeV}$ for 6 days, $3.3 \mathrm{GeV}$ for 6 days, and $4.4 \mathrm{GeV}$ for 12 days. We expect to collect true coincidence $e^{+} e^{-}$events with a rate in the range of $100-500 \mathrm{~Hz}$, providing $O(1) A^{\prime}$ signal events per minute at the threshold of sensitivity. The total $e^{+} e^{-}$sample size will exceed $10^{8}$ pairs in a 6-day period for each setting [8].

As shown in Fig. 4.4, the proposed experiment will be sensitive to $A^{\prime}$ s with couplings as small as $\alpha^{\prime} / \alpha \sim(6-8) \times 10^{-8}$ for masses in the range $65-300 \mathrm{MeV}$, and couplings as small as $\alpha^{\prime} / \alpha \sim 2 \times 10^{-7}$ for larger $m_{A^{\prime}} \lesssim 525 \mathrm{MeV}$. This is a factor of $\sim 3-35$ times lower in $\epsilon$ than existing constraints, and corresponds to $\sim 10-1000$ times smaller cross-sections [8]. 


\section{CHAPTER 5}

\section{The APEX Test Run}

The APEX experiment held a test run at Jefferson Lab in experimental Hall A from June 21st to July 12th of 2010. The purpose of the test run was to address specific PAC concerns about the experiment's proposal and to collect enough statistics to search for the $A^{\prime}$ within a significant region of parameter space.

The $A^{\prime}$ search was performed by studying $e^{+} e^{-}$production off an electron beam incident on a tantalum foil target of thickness $22 \mathrm{mg} / \mathrm{cm}^{2}$. The test run used a $2.260 \pm 0.002 \mathrm{GeV}$ electron beam with an intensity up to $150 \mu \mathrm{A}$. The central momentum of the HRSs was set to $\simeq 1.131 \mathrm{GeV}$. Dipole septum magnets allowed the detection of $e^{-}$and $e^{+}$at angles of $5^{\circ}$ relative to the incident beam.

\subsection{PAC Concerns}

APEX received conditional approval from the Program Advisory Commity (PAC) in January of 2010. We were asked to demonstrate needed performance in a two week test run. The PAC gave seven conditions that needed to be addressed in the test run [23]: 
1. Run with the zig-zag mesh design of the tungsten target and prove that it allows the requested vertex resolution.

2. Prove that it is possible to reach the uncertainty of 0.1 mrad in determining the central scattering angle between the two spectrometers.

3. Prove that the vertical drift chambers (VDCs) can operate at a rate higher than $20 \mathrm{kHz} /$ wire (That, according to the TAC report, is the maximum Hall A has operated until now).

4. Prove that it is possible to use the gas Cherenkov counters in the trigger to help clean pions. In fact, the TAC report claims that this is not possible with total rates/PMT at the level of a few hundred Hz to MHz. Also, prove that the off-line rejection of 10,000:1 can be achieved.

5. Prove that $20 \mathrm{~ns}(\mathrm{SO}-\mathrm{SO})$ and $40 \mathrm{~ns}(\mathrm{SO}-\mathrm{SO}-\mathrm{C})$ can be achieved.

6. If it is possible (not obvious for a test run), it will be advisable to set the septum magnets at higher fields to prove that also at energies higher than 2 GeV it is possible to reach the uniformity of the field requested from the experiment.

7. Provide a detailed description of different contributions to background and their importance (how assumpions and/or approximations can influence the predictions) and comparison with measurement.

\subsection{Experimental Setup}

The APEX test run took place in Hall A from June 21st, 2010 to July 12th, 2010. While it was originally scheduled only for a two week period, it was extended by a further week. 
The first week was spent removing the PREX detectors and installing the LeftHRS detector components. The detectors were comissioned using cosmic rays and low beam currents. The first optics data was collected, and high rate data was collected using the lead/diamond target.

During the second week the Right-HRS detector components were installed. The coincidence trigger electronics were installed and the DAQ was tested. After commissioning the Right-HRS detectors and setting up the coincidence triggers, production data was collected that included $e^{+} e^{-}$coincident events. Also, the VDCs were tested at rates up to $8 \mathrm{MHz}$.

The third week was crucial to the success of the test run. A sizeable amount of high quality optics data was collected for both spectrometers. Most of the week was spent collecting production data. Having this extra week enabled us to collect four million events that passed our coincidence trigger. Collecting this large data sample allowed us to search for an $A^{\prime}$ within a significant region of phase space and obtain a publishable result.

\subsubsection{Targets}

A production target was constructed and delivered to Jefferson Lab to be used in the test run. Due to a lack of technical manpower and high radiation levels, however, the target was never installed. Instead, a single tantalum foil of thickness $22 \mathrm{mg} / \mathrm{cm}^{2}$ was used for production data taking.

To test the high rate performance of the HRS detectors, a lead/diamond target was used (Fig. 5.1). This target was designed for the PREX experiment [24]. It consists of a $0.5 \mathrm{~mm}$ foil of lead sandwiched between two $0.2 \mathrm{~mm}$ sheets of diamond. 


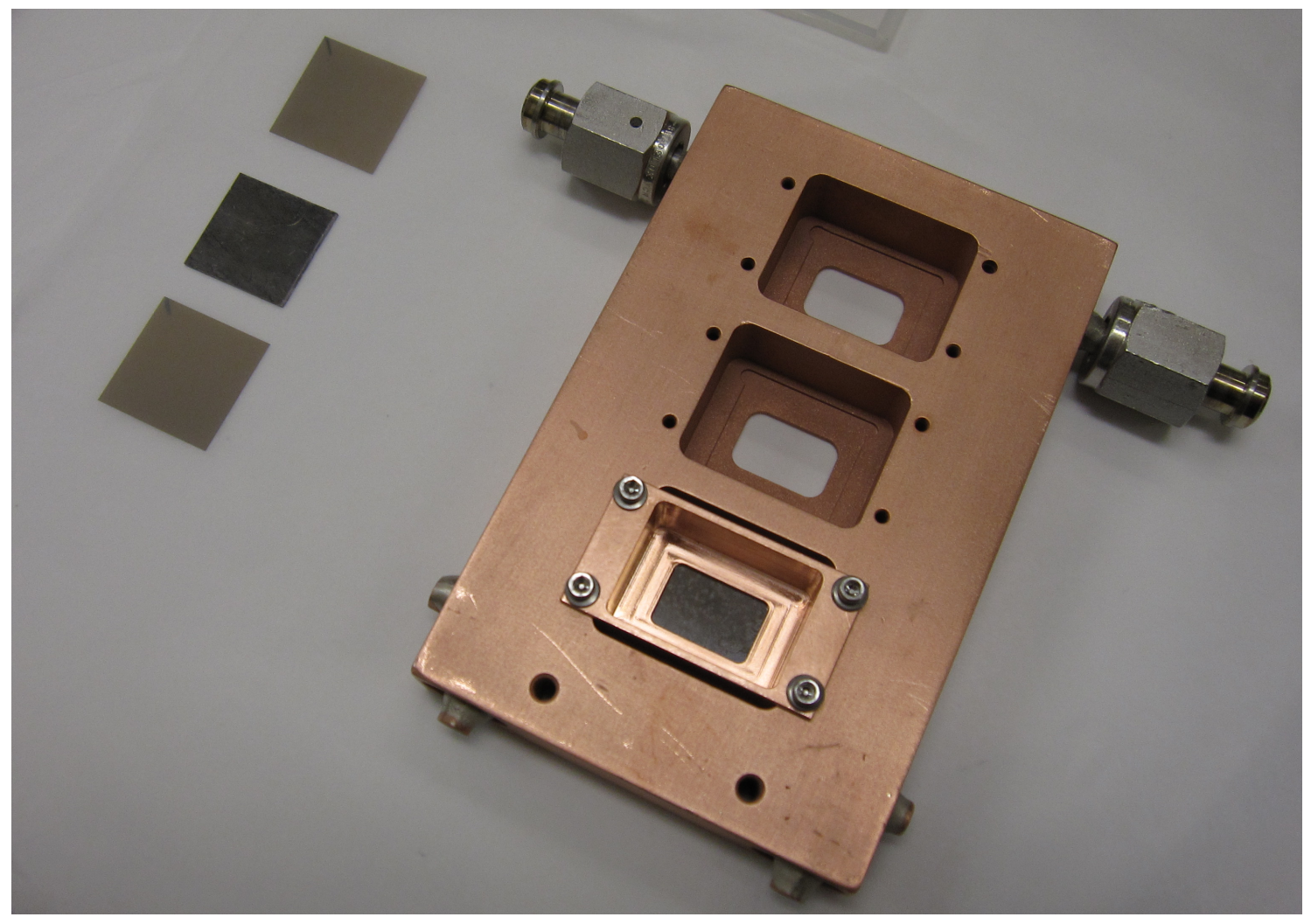

FIG. 5.1: The lead/diamond target used for high rate data taking.

\subsubsection{Septum and Beam Steering Magnets}

The HRSs can rotate to a minimum angle of $12.5^{\circ}$ with respect to the beamline. To detect $e^{+} e^{-}$pairs at a scattering angle of $5^{\circ}$, room temperature dipole septum magnets were installed between the target and opening of the spectrometers (Fig. 5.2). These septum magnets were originally designed for the PREX experiment [24].

During the test run, the polarity of the septum magnets was set to bend electrons toward the left spectrometer and positrons toward the right spectrometer. As the electron beam passed downstream of the target, the septum magnets would bend its trajectory toward the left spectrometer. Three additional magnets were installed onto the beam line to correct the beam's trajectory back to the beam dump. Two were installed upstream of the target, and the third was installed downstream. The 


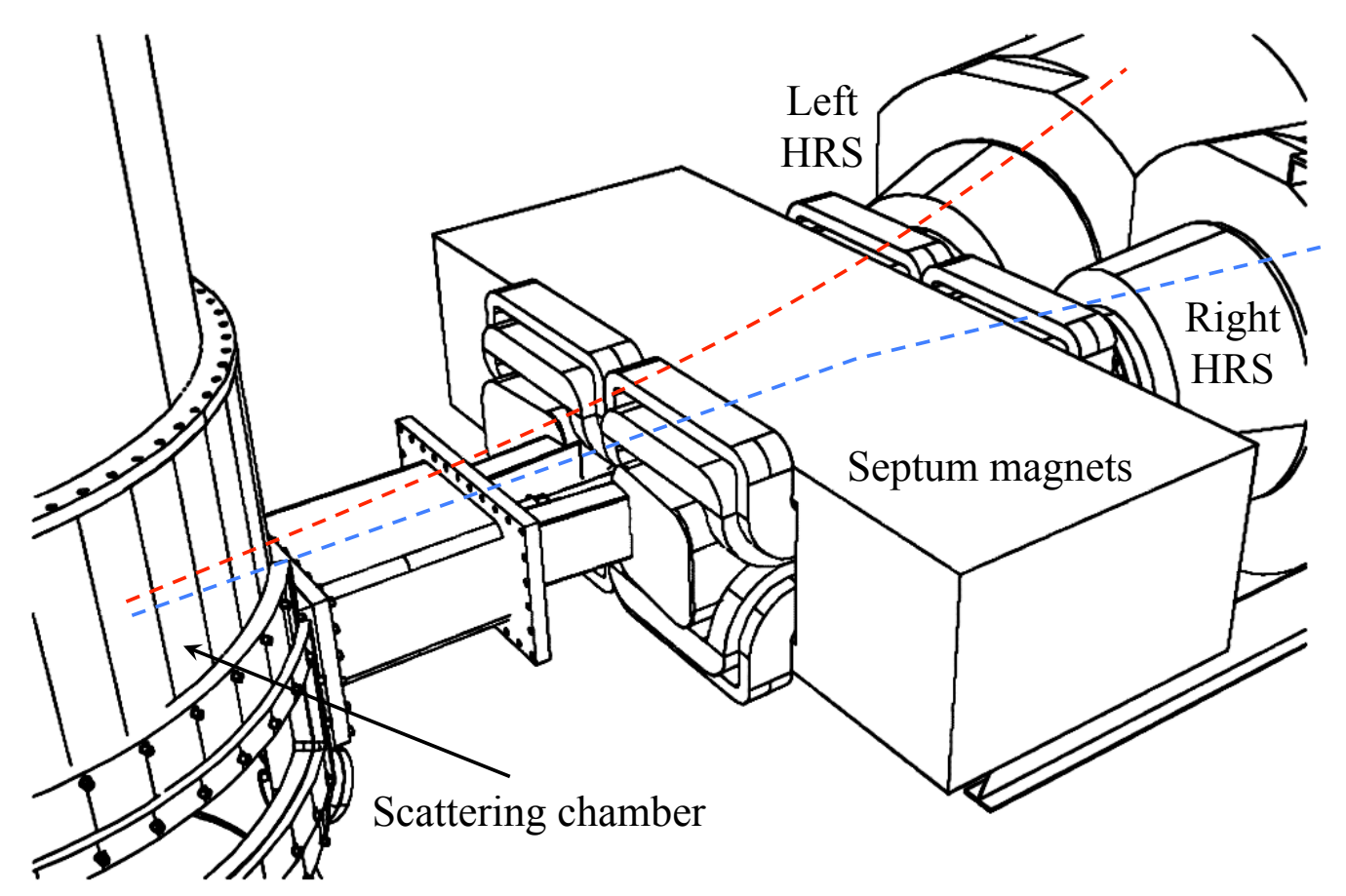

FIG. 5.2: The dipole septum magnets allow detection of $e^{-}$and $e^{+}$at angles of $5^{\circ}$ relative to the incident beam.

two that were installed upstream of the target had to be taken into account when calculating the beam position at the target.

\subsubsection{Electronics and Trigger}

The design of the front end electronics is driven primarily by the need to operate accurately at rates up to $5 \mathrm{MHz}$. The electronics system was comprised of NIM electronics, VME scalers, and fastbus Amplitude-to-Digital converters (ADCs) and Time-to-Digital converters (TDCs). To improve the high rate capabilites of the detectors, efforts were made to lower PMT high voltages and shorten the logical pulse widths. The PMT voltages for the gas Cherenkov detectors were adjusted so 
that their average single photoelectron pulse had an amplitude of $5 \mathrm{mV}$. The length of the logical pulses used for the digital electronics was kept to $10 \mathrm{~ns}$.

To improve the high rate capability of the VDCs, new amplifier/discriminator cards were used in the VDC read-out system. These cards were designed by JLab's Electronics Group to reduce the signal threshold and allow operation of the VDCs at smaller gains (see Sec. 4.4.3). With these cards installed, a high voltage of -3.5 $\mathrm{kV}$ was supplied to the VDCs instead of the nominal $-4 \mathrm{kV}$.

\section{Trigger}

The APEX test run used seven different triggers, labeled T1-T6 and T8. T1 and T3 were a logical OR of the 16 PMTs on the right side of the S2m plane in the left and right spectrometers, respectively. T2 was taken from the top PMT of the S0 counter in the left HRS. T5 was based on a 40 ns coincidence window from the left HRS S0 and gas Cherenkov detectors.

T4 and T6 were used to record coincidences between the left and right spectrometers. T4 corresponded to a coincidence between the left and right S2m planes, and T6 to a coincidence between T4 and the gas Cherenkov detector in the right HRS (used to detect positrons). The gas Cherenkov detector in T6 was used to remove the pion background. T8 was a $1024 \mathrm{~Hz}$ clock used to normalize rates recorded in the scalers.

Table 5.1 summarizes the triggers used. Fig. 5.3 shows a schematic of the logic used to construct the trigger electronics. Most of the trigger components reside on the left HRS due to the location of the trigger supervisor. This ensured that the delay of the cables and distortion of the pulses were minimized. 
TABLE 5.1: Triggers of the APEX test run.

\begin{tabular}{lc}
\hline T1 & LHRS S2m \\
T2 & LHRS S0 \\
T3 & RHRS S2m \\
T4 & LHRS S2m AND RHRS S2m \\
T5 & LHRS S0 AND LHRS Gas Cherenkov \\
T6 & T4 AND RHRS Gas Cherenkov \\
\hline \hline
\end{tabular}

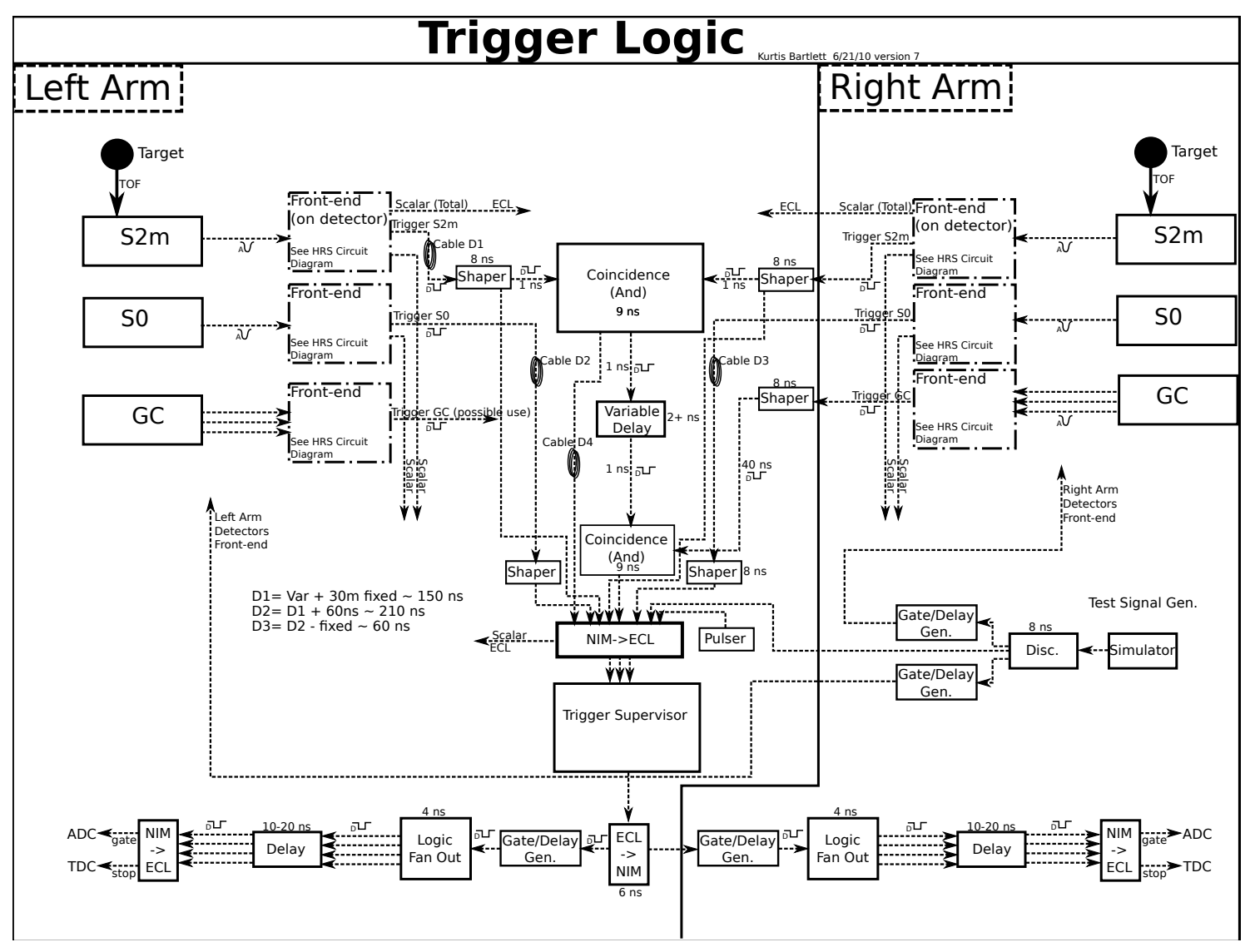

FIG. 5.3: Schematic of the trigger electronics. 


\section{CHAPTER 6}

\section{Detector Calibrations}

The detectors used in any experiment must be calibrated in order to make accurate measurements. This chapter will cover the detector calibrations performed during the APEX test run.

Determining the invariant mass of $e^{+} e^{-}$pairs requires a careful understanding of the reconstructed beam position at the target, as well as the reconstruction of the $e^{-}$and $e^{+}$trajectories inside the spectrometers. Due to the high luminosity of APEX, additional considerations must be made when calibrating the detectors to ensure accurate detector and data acquisition performance at high rates. In this chapter we will discuss the calibration of the BPMs and HRS detectors used during

the test run. We will also examine the magnetic optics calibration of the HRSs that allows us to achieve precise track reconstruction. 


\subsection{HRS Detectors}

\subsubsection{Scintillators}

The S2m scintillator planes were used for timing and trigger purposes during the APEX test run. Both S2m planes were used along with the Right-HRS gas Cherenkov counter for the final coincidence trigger to select true $e^{+} e^{-}$pairs. Because of the high rates experienced by the detectors during the experiment, it was important to use a small coincidence timing gate in order to avoid recording an excess of accidental coincidence events. To achieve a small enough timing gate, the timing of the PMT signals must be aligned in each scintillator plane.

A logical OR between the left PMTs of each S2m plane was used as part of the coincidence trigger. Therefore, it was only necessary to align the timing of the left PMTs of each plane. LeCroy 1877 TDCs were used to measure the timing of the PMT signals. These TDCs have $0.5 \mathrm{~ns}$ resolution and were operated in common stop mode. To align the timing of the PMTs, electrons were scattered off a lead/diamond target in order to provide sufficient statistics. The S0 scintillator counter was used as a timing reference, and 1-5 ns delay cables were inserted to adjust the timing of each PMT. Fig. 6.1 demonstrates an example of the timing spread that was achieved during the test run.

The amount of charge in a PMT signal produced for a fixed amount of light depends on the gain of the PMT. The gain can be controlled by adjusting the highvoltage (HV) supplied to the PMT. The HV supplied to the S2m PMTs must be adjusted so that gain of each PMT is the same. The gain matching was done using cosmic ray data. Events were selected from the center of the paddles using TDC information from the VDCs. The ADC distributions were plotted and the first photoelectron peaks were fit with a Gaussian for each PMT. The HV of each PMT 


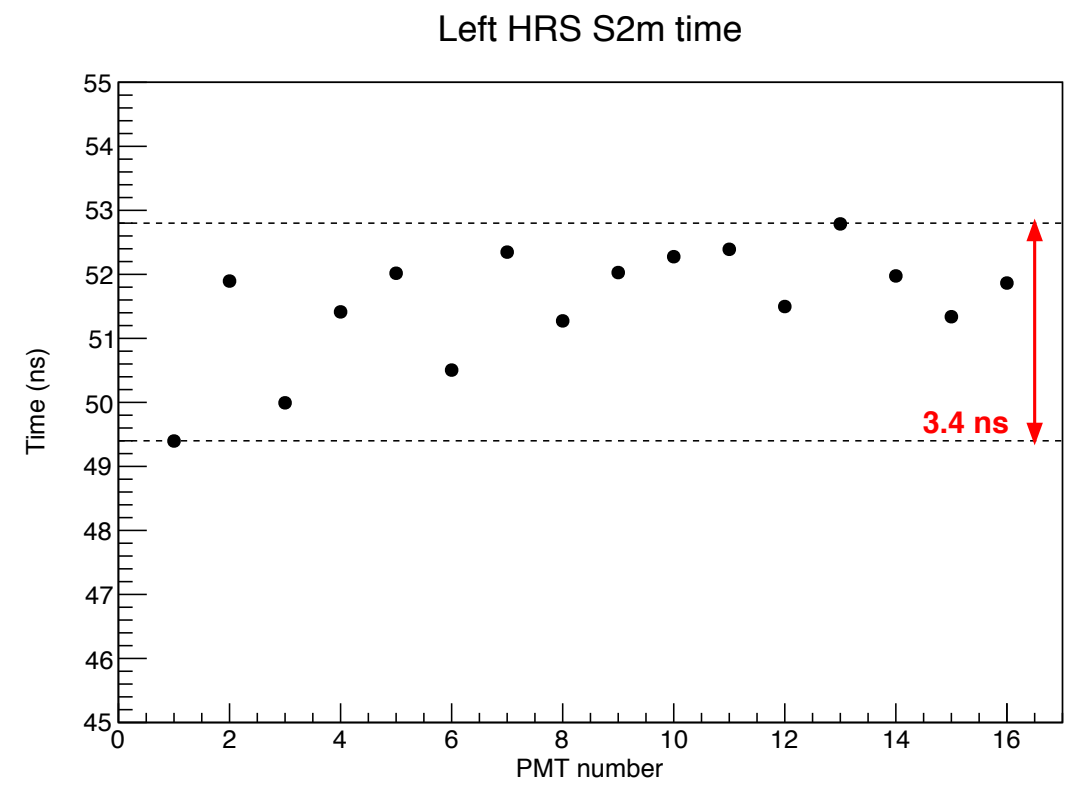

FIG. 6.1: The average timing of the PMT signals for the Left-HRS S2m plane after inserting 1-5 ns delay cables. The S0 counter was used as a reference. A timing spread of $\sim 3.5$ ns was achieved.

was then adjusted using

$$
H V_{\text {new }}=\sqrt[10]{\frac{20}{A D C_{\text {peak }}-A D C_{\text {pedestal }}}} \times H V_{\text {old }}
$$

where $A D C_{\text {peak }}$ is the centroid of the gaussian fit and $A D C_{\text {pedestal }}$ is the pedestal value. The pedestal represents the output of an ADC channel when there is no signal at its input. The pedestal acts as a starting point from which the ADC output starts counting. Thus, the pedestal must be subtracted from the raw ADC value to obtain the true $\mathrm{ADC}$ readout corresponding to the size of the input signal. The pedestal locations vary for each channel, and need to be determined for each channel separately. 


\subsubsection{Gas Cherenkov Detector}

The gas Cherenkov detector in the Right-HRS was used as part of the coincidence trigger to provide trigger-level $\pi^{+}$rejection. Just as for the S2m scintillator planes, it was essential that all ten PMTs in the detector had the same timing and gain. The timing alignment and gain matching of the gas Cherenkov PMTs was done using the same procedures described in Sec. 6.1.1.

The ADC spectrum of the gas Cherenkov detector was used to select the final event sample, so a more detailed off-line ADC calibration was needed. The sum

of the amplitudes of all ten PMT signals was used to separate the $e^{+}$from meson background (see Sec. 7.2). Positrons were identified by making a cut above the sum of the first photoelectron peaks for all ten PMTs. Therefore, it was essential to align the first photoelectron peak of each PMT.

The first photoelectron peaks were aligned by applying amplitude transformation coefficients to the ADC spectrums. The first photo electron peaks were each fit with a Gaussian (as in Sec. 6.1.1), and the corrected ADC values were calculated using

$$
A_{i}^{\text {corr }}=\left(A D C_{\text {peak }}-A D C_{\text {pedestal }}\right) \times A_{i}^{\text {raw }}
$$

where $A D C_{\text {peak }}$ is the centroid of the Gaussian fit, $A D C_{\text {pedestal }}$ is the pedestal value, and $A_{i}^{\text {raw }}$ is the uncorrected ADC value. Fig. 6.2 shows the sum ADC spectrum before and after the correction.

\subsubsection{Drift Chambers}

\section{Time offsets}

The vertical drift chambers are responsible for reconstructing the $e^{-}$and $e^{+}$ tracks inside the spectrometers. When a charged particle passes through the drift 


\section{Gas Cherenkov Amplitude Sum}

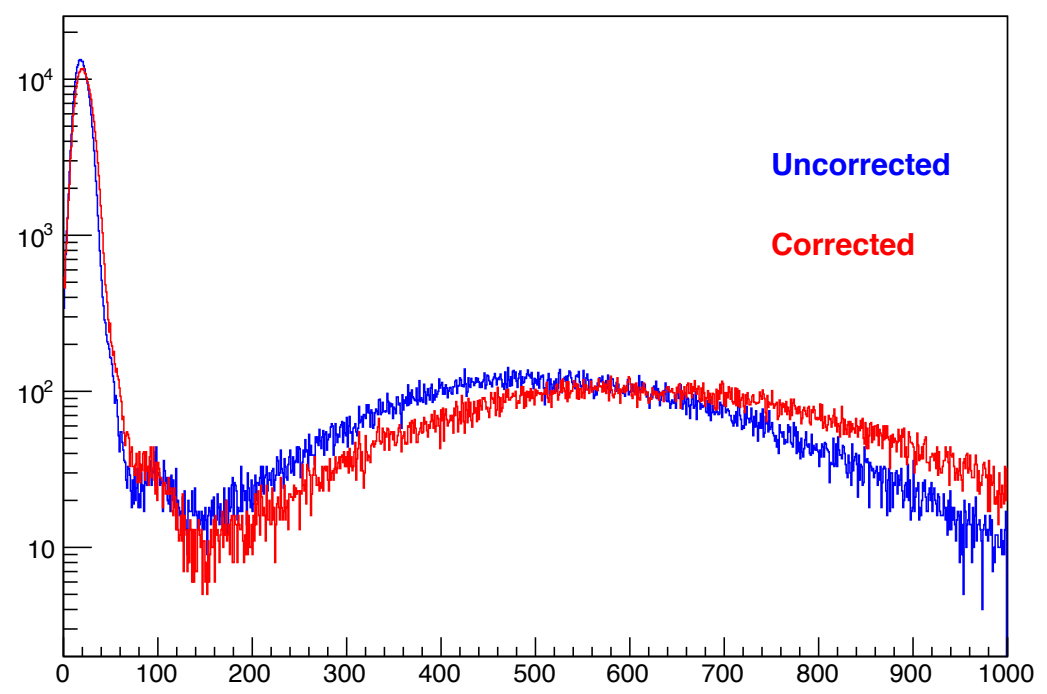

FIG. 6.2: The sum of all ten gas Cherenkov PMT amplitudes before and after off-line correction.

chamber it produces ionization. The resulting ions and electrons are accelerated by the electric field surrounding the nearest wire, producing an electric current on the wire. TDCs are used to measure the timing of the resulting signal relative to the timing of the trigger. The time for which it takes the ionization to drift to the wire is called the drift time, and can be extracted from the time measured by the TDC. The readout time for each wire, $i$, can be expressed

$$
t_{\mathrm{TDC}}=t_{\mathrm{drift}}+t_{\mathrm{delay}, i}-t_{\mathrm{path}}-t_{\mathrm{trig}} \approx t_{\mathrm{drift}}+t_{0, i}
$$

where $t_{\text {drift }}$ is the drift time, $t_{\text {delay }, i}$ is the time it takes for the signal to travel to the TDC and is unique for each wire, $t_{\text {path }}$ is the time it takes for the electron to travel from the drift chamber to the trigger detectors, and $t_{\text {trig }}$ is the time it takes for the trigger to occur and be sent to the common stop of the TDC [25]. The last three terms can be grouped into a single offset, $t_{0, i}$.

The wire signals travel to amplifier/discriminator (A/D) cards before going to 


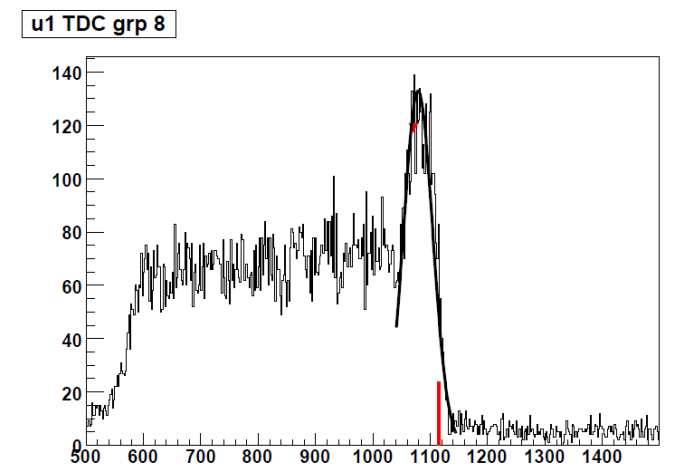

(a)

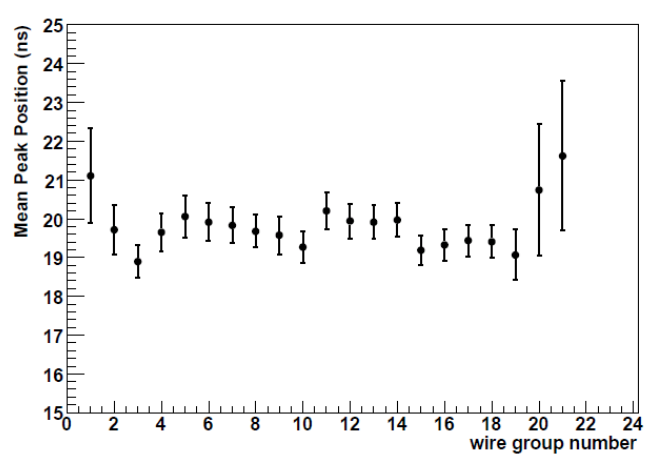

(b)

FIG. 6.3: An example of a raw TDC spectrum for a group of 16 wires is shown in (A). The histogram is backwards in time because the TDCs were operating in common stop mode. The peak towards the right corresponds to the sudden movement of charged particles near the wire. To determine the time offset $t_{0}$, the peak is fit with a gaussian and $t_{0}$ is fixed at $1.4 \sigma$ to the right of the peak (red line). (B) compares $t_{0}$ for each group.

the input of the TDCs. Each A/D card takes signals from 16 wires and sends the output along a ribbon cable to the TDC. Because these 16 wires use the same A/D card and travel along the same length of ribbon cable, it is sufficient to determine a single time offset $t_{0}$ for the entire group of 16 . An example of a typical VDC time spectrum is shown in Fig. 6.3a. Since the TDCs were operating in common stop mode, the histogram is backwards in time. The peak on the right corresponds to the sudden movement of charged particles near the wire, where as the rest of the spectrum corresponds to the movement of particles located further from the wire. To calibrate the time offset of a group of wires, the peak is fit with a gaussian and the offset is fixed at $1.4 \sigma$ to the right of the peak position. Fig. 6.3b shows the time offset determined for each group of wires. 


\subsection{Beam Position}

An accurate determination of the beam position at the target is essential for APEX. During the experiment the beam position needs to be monitored in order to ensure that it stays within the target area. If the beam strays outside of the target area it could damage the apparatus. Furthermore, the precise knowledge of the beam position at the target for every event is important for the optics calibration of the spectrometers.

Two beam position monitors (BPMs) located upstream of the target are used to determine the position of the beam. The BPM signals are read out by ADCs, the output of which can be related to position through a linear transformation. To perform this transformation one must determine the $\mathrm{x}$ and $\mathrm{y}$ position offsets and gain coefficients. These parameters are calibrated with respect to a set of wire scanners located adjacent to the BPMs.

There is a difference of a few $\mu$ s from the time when the particle hits the target and the time when the BPMs return the corresponding beam position [26]. Therefore, when using a rastered beam the BPMs alone are not sufficient for determining the beam position at the target for each event. To account for this, the current information of the two raster magnets is used in addition to the BPM information to determine the event-by-event on-target beam position. The beam position is calculated by

$$
x_{t g}^{\text {raster }}=O_{x}+A_{x} \mathrm{I}_{x}^{\text {raster }}, \quad y_{t g}^{\text {raster }}=O_{y}+A_{y} \mathrm{I}_{y}^{\text {raster }},
$$

where $O_{x}$ and $O_{y}$ are the offset corrections, $A_{x}$ and $A_{y}$ are the transformation coefficients, and $\mathrm{I}_{x}^{\mathrm{raster}}$ and $\mathrm{I}_{y}^{\text {raster }}$ are the currents in each of the raster magnets. The transformation coefficients are calculated from the widths of the BPM and current 

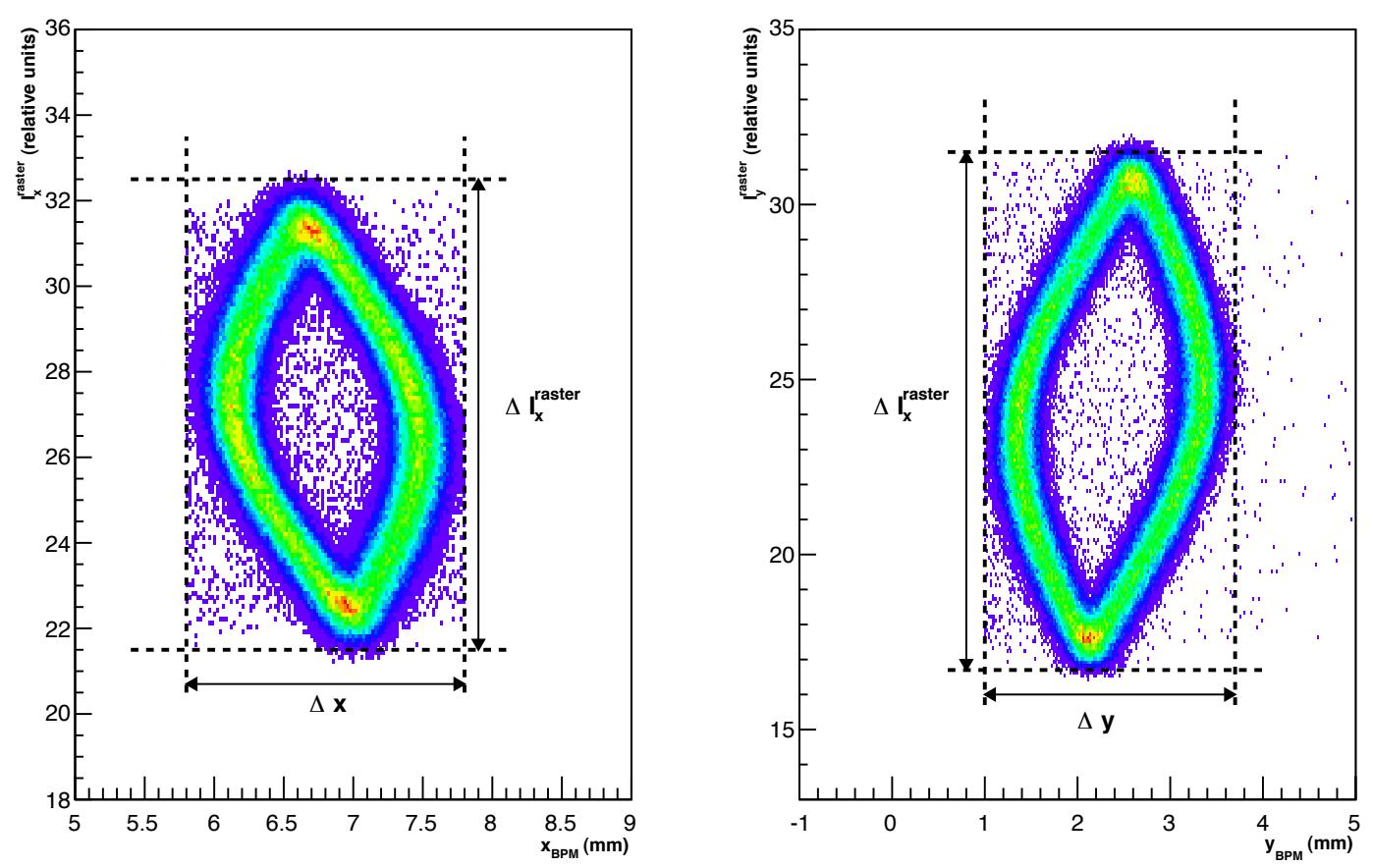

FIG. 6.4: The raster current vs. the horizontal beam position measured by the BPMs. The widths of the distributions are used to calculate the corrected beam position. Note the $\pi / 2$ phase difference between the $\mathrm{x}$ and $\mathrm{y}$ plots.

distributions (see Fig. 6.4):

$$
A_{x}=\frac{\Delta x_{B P M}}{\Delta \mathrm{I}_{x}^{\text {raster }}}, \quad A_{y}=\frac{\Delta y_{B P M}}{\Delta \mathrm{I}_{y}^{\text {raster }}}
$$

The offsets are calculated by comparing the average beam position measured by the BPMs to the average reconstructed beam position from the raster current:

$$
O_{x}=\bar{x}_{\mathrm{BPM}}-\overline{\mathrm{I}}_{x}^{\mathrm{raster}} A_{x}, \quad O_{y}=\bar{y}_{\mathrm{BPM}}-\overline{\mathrm{I}}_{y}^{\mathrm{raster}} A_{y} .
$$

After all the parameters are calibrated they are stored in a database file that is used to create the corrected beam position variables. 


\section{Additional beamline magnets}

The septum magnet was used to bend negatively charged particles toward the left-HRS and positively charged particles toward the right-HRS. As the electron beam travels downstream of the target the septum magnet bends its trajectory towards the left-HRS. Three additional magnets were installed onto the beam line to correct the beam's trajectory back to the beam dump. Two of the magnets were installed upstream of the target, so their bending effects must be taken into account when determining the on-target beam position.

The bending of the beam can be calculated using the field integral of each magnet $\left(\int B d l\right)$, the momentum of the beam $(p)$, and the distance from the magnet to the target (d):

$$
\Delta x=\mathrm{d} \times \theta=\mathrm{d} \times \frac{\int B d l}{p} .
$$

The final beam position at the target was shifted toward the Left-HRS by $2.9 \pm 0.2$ $\mathrm{mm}$ due to the bending of the additional beam line magnets. This value was inserted into a database file used to create the variables for the final on-target beam position.

\subsection{Optics Calibration}

Reconstructing the invariant mass of $e^{+} e^{-}$pairs requires the precise determination of the position and angle of the particles at their reaction vertex. The focal plane coordinates of a detected particle are measured directly by the vertical drift chambers (VDCs). The spectrometer's optics matrix establishes a mapping between the focal plane coordinates and the interaction vertex. The optics matrix elements must be calibrated to provide an accurate and precise measurement of the vertex of the $e^{+} e^{-}$pairs. This section will cover the optimization of these matrix elements. 


\subsubsection{Coordinate Systems}

A detailed description of the coordinate systems used in the reconstruction of trajectories is given below. Fig. 6.5 illustrates the orientation of each coordinate system.

\section{Hall Coordinate System}

The origin of the Hall Coordinate System (HCS) is at the center of the hall, which is defined by the intersection of the beam axis and the vertical symmetry axis of the target system. The positive $\hat{z}$ direction is along the beam axis towards the beam dump, and the positive $\hat{y}$ direction is vertically up.

\section{Target Coordinate System}

Each spectrometer has its own Target Coordinate System (TCS). The positive $\hat{z}_{t g}$ direction is along a line perpendicular to the sieve plane that passes through the central sieve hole. In the ideal case, where the spectrometer is pointing directly at the center of the hall and the sieve slit is perfectly centered on the spectrometer, $\hat{z}_{t g}$ points directly at the hall center. For this case, $Z_{0}$ is defined as the distance from the hall center to the central sieve hole. The origin of the TCS is defined as the point on the $\hat{z}_{t g}$ axis that is a distance $Z_{0}$ from the sieve surface. The positive $\hat{x}_{t g}$ direction is parallel to the sieve plane, pointing vertically down. The out-of-plane angle $\left(\theta_{t g}\right)$ and in-plane-angle $\left(\phi_{t g}\right)$ are given by $\frac{\mathrm{d} x}{Z_{0}}$ and $\frac{\mathrm{d} y}{Z_{0}}$ respectively.

\section{Detector Coordinate System}

In the lower VDC, the intersection of wire 184 of the U1 plane and wire 184 of the V1 plane defines the origin of the Detector Coordinate System (DCS). In this coordinate system, $\hat{y}$ is parallel to the short symmetry axis of the lower VDC, and 
$\hat{x}$ is parallel to the long symmetry axis pointing away from the center of curvature of the dipole. The positive $\hat{z}$ direction is vertically up.

\section{Transport Coordinate System}

The Transport Coordinate System (TRCS) is generated by rotating the DCS clockwise around the $y$-axis by $45^{\circ}$. In the TRCS the trajectory of a particle can be represented by a vector

$$
\vec{x}=\left[\begin{array}{c}
x \\
\theta \\
y \\
\phi \\
\delta
\end{array}\right]
$$

where $x$ is the displacement of the trajectory in the dispersive plane relative the central reference trajectory (vertical direction for the HRS), $\theta$ is the tangent of the angle the trajectory makes in the dispersive plane, $y$ and $\phi$ are the same as $x$ and $\theta$ in the transverse plane (horizontal direction for the $\operatorname{HRS}$ ), and $\delta(\Delta p / p)$ is the fractional momentum of the trajectory from the central momentum setting of the spectrometer.

\subsubsection{General Approach}

The variables $x_{d e t}, \theta_{d e t}, y_{d e t}$, and $\phi_{d e t}$ are measured by the VDCs and are used to calculate the $x, \theta, y, \phi$, and $\delta$ variables at the target. The $x_{t g}$ value can be fixed at zero during optics calibration by requiring the on-target beam position to be within $250 \mu \mathrm{m}$ of the origin of the HCS. As a first-order approximation the relationship 


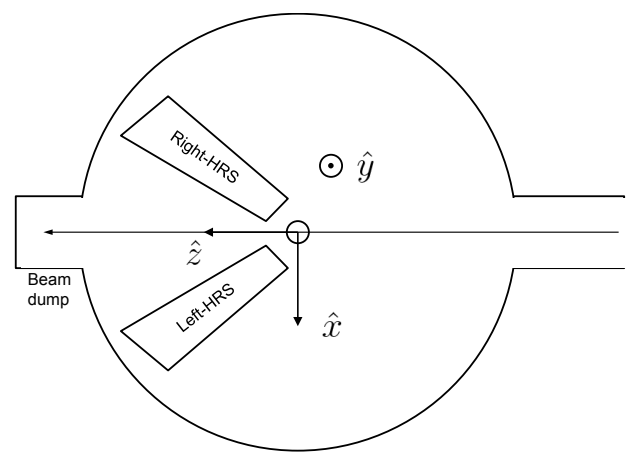

(a) Hall Coordinate System

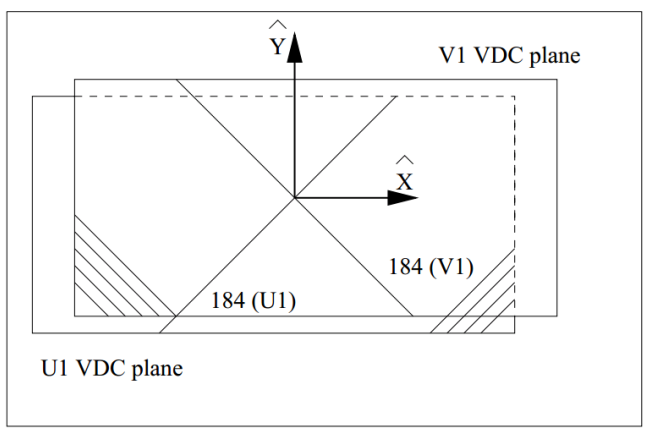

(c) Detector Coordinate System

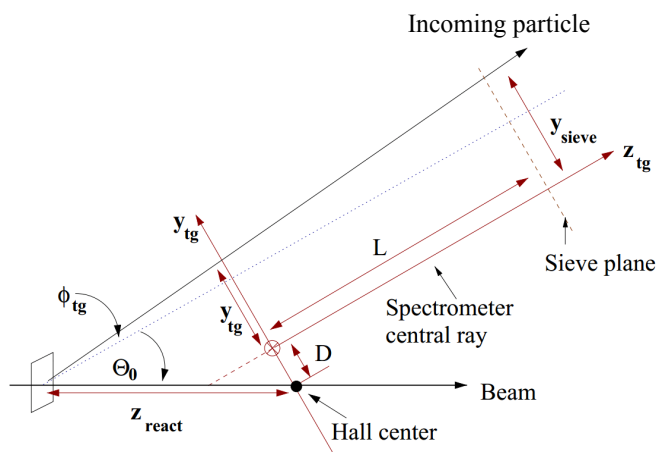

(b) Target Coordinate System

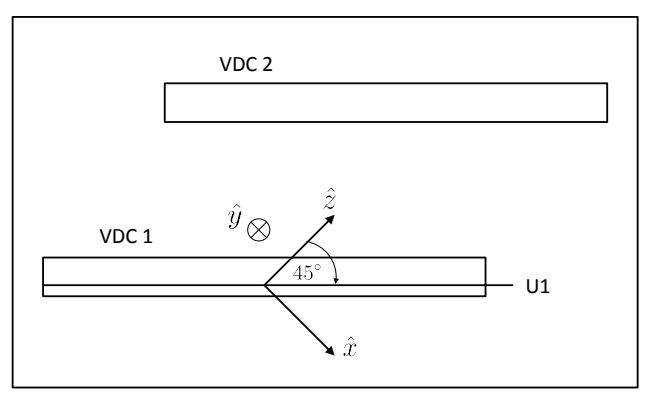

(d) Transport Coordinate System

FIG. 6.5: Hall A coordinate systems.

between the focal plane and target coordinates can be written [27]

$$
\left[\begin{array}{l}
\delta \\
\theta \\
y \\
\phi
\end{array}\right]_{t g}=\left[\begin{array}{cccc}
\langle\delta \mid x\rangle & \langle\delta \mid \theta\rangle & 0 & 0 \\
\langle\theta \mid x\rangle & \langle\theta \mid \theta\rangle & 0 & 0 \\
0 & 0 & \langle y \mid y\rangle & \langle y \mid \phi\rangle \\
0 & 0 & \langle\phi \mid y\rangle & \langle\phi \mid \phi\rangle
\end{array}\right]\left[\begin{array}{c}
x \\
\theta \\
\phi \\
y
\end{array}\right]_{f p}
$$

where the bra-ket notation denotes the scalar product between directional unit vectors, e.g. $\langle\alpha \mid \beta\rangle \equiv \hat{\alpha} \cdot \hat{\beta}$. The zero tensor elements result from the mid-plane symmetry of the spectrometer.

The focal plane coordinates are linked to the target coordinates through a set 
of tensors $Y_{j, k, l}, T_{j, k, l}, P_{j, k, l}$, and $D_{j, k, l}$ according to [27]

$$
\begin{gathered}
y_{t g}=\sum_{j, k, l} Y_{j, k, l} \theta_{f p}^{j} y_{f p}^{k} \phi_{f p}^{l}, \\
\theta_{t g}=\sum_{j, k, l} T_{j, k, l} \theta_{f p}^{j} y_{f p}^{k} \phi_{f p}^{l}, \\
\phi_{t g}=\sum_{j, k, l} P_{j, k, l} \theta_{f p}^{j} y_{f p}^{k} \phi_{f p}^{l}, \\
\delta=\sum_{j, k, l} D_{j, k, l} \theta_{f p}^{j} y_{f p}^{k} \phi_{f p}^{l},
\end{gathered}
$$

where the superscripts denote the power of each focal plane variable. The tensors $Y_{j, k, l}, T_{j, k, l}, P_{j, k, l}$, and $D_{j, k, l}$ are polynomials in $x_{f p}$. For example, $Y_{j, k, l}$ can be expressed

$$
Y_{j, k, l}=\sum_{i=1}^{m} C_{i}^{Y_{j, k, l}} x_{f p}^{i},
$$

so the final expression for $y_{t g}$ is

$$
y_{t g}=\sum_{j, k, l} \sum_{i=1}^{m} C_{i}^{Y_{j, k, l}} x_{f p}^{i} \theta_{f p}^{j} y_{f p}^{k} \phi_{f p}^{l} .
$$

The coefficients $C_{i}^{Y_{j, k, l}}$ are the optics matrix elements which are stored in a database used to reconstruct the target variables.

\subsubsection{Procedure}

The transport tensors are optimized by performing a $\chi^{2}$ minimization on the difference between the reconstructed target variables and the actual target variables. In practice, however, it is difficult to obtain the actual values for the basic target variables $y_{t g}, \theta_{t g}$, and $\phi_{t g}$. Instead, the optics matrix elements are calibrated by using a sieve slit collimator. The sieve slit is a removable 0.25 inch-thick tungsten plate 
with a grid of holes drilled through at known positions, and is inserted in front of the entrance of the spectrometer during a calibration run (see Fig. 6.6). Electrons lose enough energy when passing through the sieve plate so that only electrons with trajectories passing through the sieve holes reach the detectors. The horizontal and vertical positions of the scattered electron in the sieve plane, $x_{\text {sieve }}$ and $y_{\text {sieve }}$, are uniquely determined for quasi-elastic scattered electrons. The basic target variables can be used to calculate the sieve plane variables using the equations

$$
\begin{aligned}
& x_{\text {sieve }}=x_{t g}+L \tan \theta_{t g}, \\
& y_{\text {sieve }}=y_{t g}+L \tan \phi_{t g},
\end{aligned}
$$

where $L$ is the distance from the hall center to the sieve plane. The vertical coordinate $x_{t g}$ is obtained using the BPMs. The optics matrix elements are optimized by minimizing the following function:

$$
\begin{aligned}
\chi_{x}^{2} & =\sum_{\mathrm{i}=0}^{\text {Events }}\left(x_{\text {sieve }}^{i}-x_{\text {sieve }}^{0}\right)^{2} \\
\chi_{y}^{2} & =\sum_{\mathrm{i}=0}^{\text {Events }}\left(y_{\text {sieve }}^{i}-y_{\text {sieve }}^{0}\right)^{2},
\end{aligned}
$$

where $x_{\text {sieve }}^{0}$ and $y_{\text {sieve }}^{0}$ correspond to the surveyed location of the sieve hole. Only 1000 events are selected for each hole in order to avoid any bias across the full acceptance.

The momentum calibration is performed by scanning the central momentum of the spectrometer $p_{0}$ around the elastic peak, i.e., $\delta$-scans at $p_{0}=p_{\text {elastic }}, \pm 1 \%, \pm 2 \%, \pm 3 \%$, and $\pm 4 \%$. The real momentum is calculated using the scattering angle and the corrections due to the radiative energy losses in the target material. The $\delta$ matrix elements are optimized by performing a global fit on data from all $\delta$-scan points. 


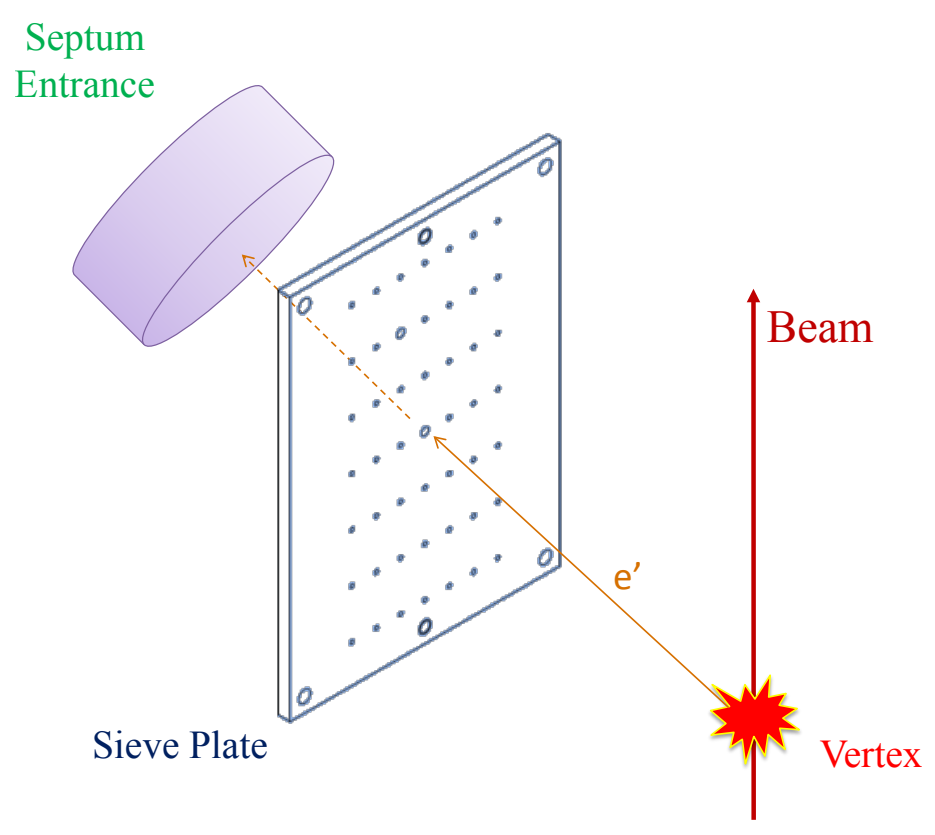

FIG. 6.6

FIG. 6.7: During the angular calibration sieve slits were inserted between the reaction vertex and the entrance to the septum magnet [28]. Only the electrons whose trajectories pass through the sieve holes make it to the detectors. The optics matrix elements can be optimized by comparing the reconstructed holes locations with the surveyed hole locations.

The full APEX experiment will use a target consisting of multiple tungsten ribbons located along the beam axis. Therefore, it is important to calibrate the reconstruction of the reaction vertex, $z_{\text {react }}$. The reaction vertex can be calculated using the equation

$$
z_{\text {react }}=-\left(y_{t g}+D\right) \frac{\cos \phi_{t g}}{\sin \theta_{0}+\phi_{t g}}+x_{\mathrm{bpm}} \cot \theta_{0}+\phi_{t g}
$$

where $\mathrm{D}$ is the horizontal displacement of the spectrometer from its ideal position and $t$ thet $a_{0}$ is the central angle of the spectrometer. The vertex calibration is done using data from deep inelastic scattering on a multi-carbon foil target. Like the calibration procedure described above, the $\delta_{t g}$ optics matrix elements are optimized 


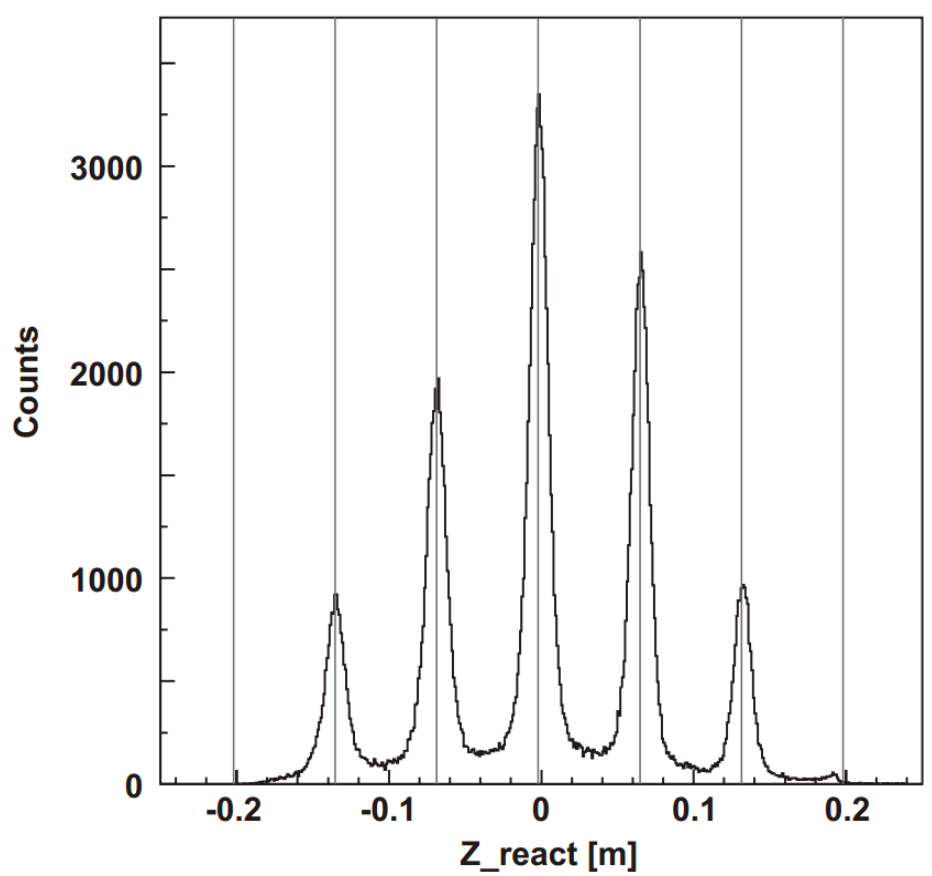

FIG. 6.8: An example of the reconstructed foil positions along the beam line after performing the vertex calibration (plot taken from [3]). The black lines indicate the surveyed positions of the foils.

using $\chi^{2}$ minimization. Fig. 6.8 shows an example of the reconstructed foil vertex peaks after calibration.

\subsubsection{Results}

During the APEX test run the optics calibration was done by Jin Huang and Vincent Sulkosky. All calibration data used a $1.162 \mathrm{GeV}$ electron beam.

The angular calibration used elastic scattering on a tantalum foil target with the sieve slits inserted in front of the entrance of the septum magnets. Calibrating the full coverage of the HRS momentum acceptance is normally done by scanning the HRS central momentum setting from $\delta \approx-4 \% \sim+4 \%$. However, due to limitations of the septum magnet current, it was only possible to scan the $\delta \approx 0 \% \sim+4 \%$ acceptance region. To calibrate the full momentum acceptance, the elastic radiative tails of $\delta \approx 0 \%$ data was used. Table 6.1 summarizes the data used to perform 
TABLE 6.1: Summary of the angular calibration data sets.

\begin{tabular}{ccc} 
Left-HRS & \\
\hline \hline Data set & Run number & $\delta$ \\
\hline 0 & 1170 & $0 \%$ with elastic peak \\
1 & 1172 & $+1 \%$ with elastic peak \\
2 & 1181 & $+2 \%$ with elastic peak \\
3 & 1183 & $+3 \%$ with elastic peak \\
4 & 1190 & $+4 \%$ with elastic peak \\
5 & $1169-1170$ & $-3 \% \sim-1 \%$ with elastic tail \\
6 & $1169-1170$ & $-4 \% \sim-3 \%$ with elastic tail \\
\hline \hline
\end{tabular}

\section{Right-HRS}

\begin{tabular}{ccc}
\hline \hline Data set & Run number & $\delta$ \\
\hline 0 & 1898 & $0 \%$ with elastic peak \\
1 & 1911 & $+2 \%$ with elastic peak \\
2 & 1920 & $+4 \%$ with elastic peak \\
3 & $1898-1899$ & $-2 \% \sim-1 \%$ with elastic tail \\
4 & $1898-1899$ & $-3.5 \% \sim-2 \%$ with elastic tail \\
\hline \hline
\end{tabular}

the angular calibration. The matrix elements for each spectrometer were optimized for all data sets simultaneously. The reconstructed sieve holes after calibration are shown in Fig. 6.9. The final horizontal (vertical) angular resolutions achieved were 0.29 (1.86) mrad for the Left-HRS and 0.44 (1.77) for the Right-HRS. See sec. 7.6.1 for details on determining the angular resolution.

The momentum calibration used the same elastic data as the angular calibration. Although the momentum could only be calibrated for $\delta \approx 0 \% \sim+4 \%$, the reach of the calibration was extended to negative $\delta$ through a linear extrapolation of the optics database used for the pentaquark search E04-012 experiment. Despite the lack of negative / delta data, the extrapolation of the existing $\delta$ matrix elements allowed us to achieve a relative momentum resolution of $<5 \times 10^{-4}$ (Fig. 6.10). The relative momentum resolution has a small contribution to the mass resolution, so this result is sufficient.

Since the test run used a single foil target, a good vertex resolution is not heavily 
demanded. The vertex position was roughly calibrated for both spectrometers using the tantalum foil and multi-carbon foil targets. The HRS central momentum setting was set such that $\delta \approx-10 \%$. The vertex resolution achieved for the tantalum foil target was $27 \mathrm{~mm}$ for the Left-HRS and $17 \mathrm{~mm}$ for the Right-HRS. 

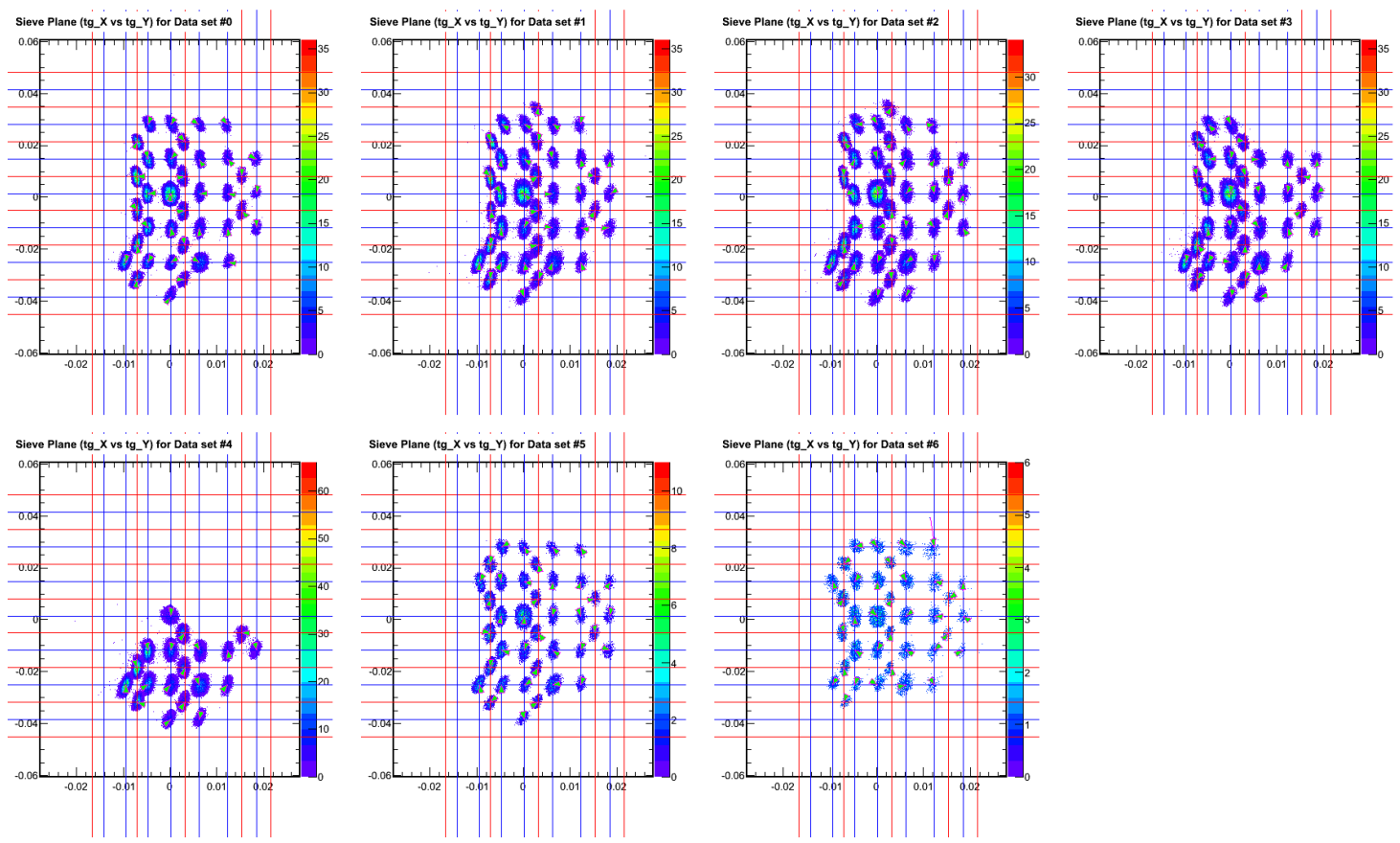

(a) Left-HRS
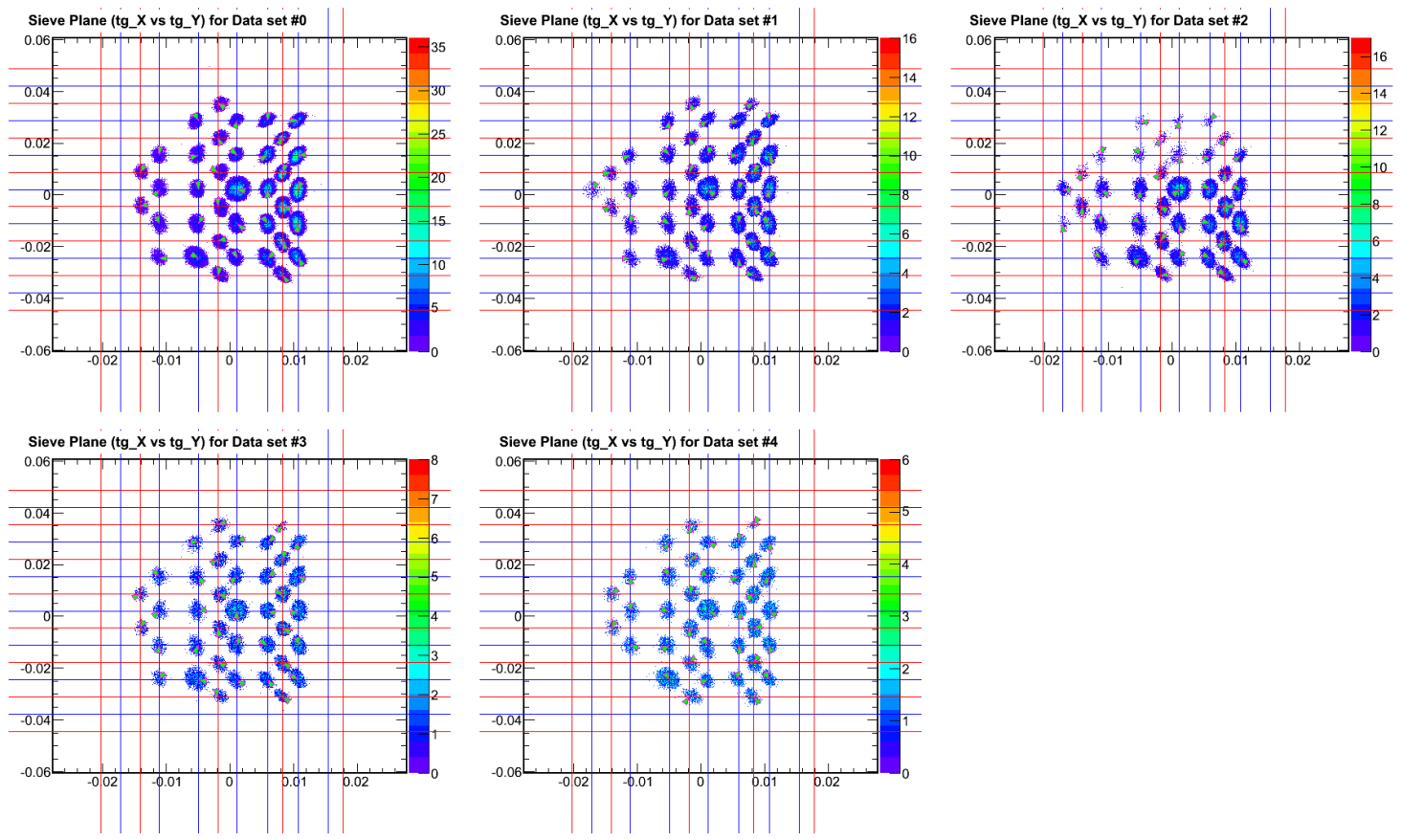

(b) Right-HRS

FIG. 6.9: Reconstructed sieve holes for all data sets (see Table 6.1) after calibration. The red and blue lines correspond to the surveyed sieve hole locations. 


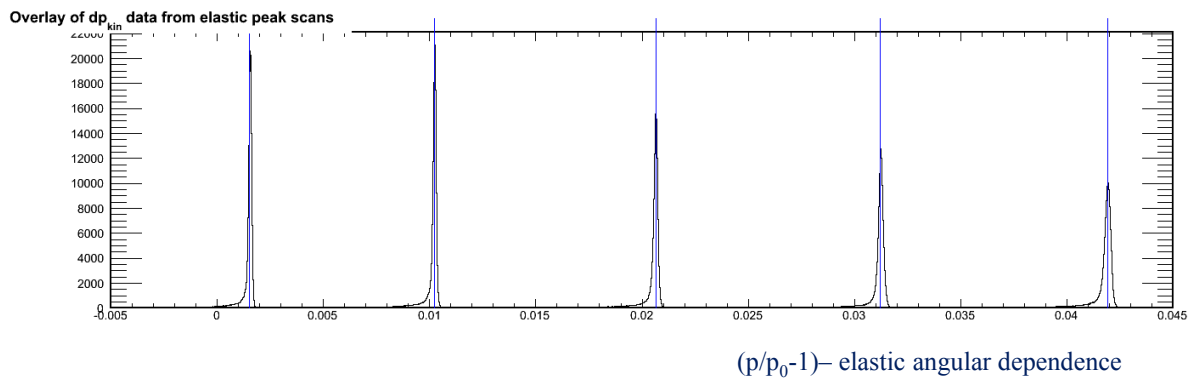

FIG. 6.10: An overlay of the $\delta$-scans for the Left-HRS after calibration. The final relative momentum resolution achieved was $<5 \times 10^{-4}$. 


\section{CHAPTER 7}

\section{Data Analysis}

The ultimate goal of the data analysis is to generate the final invariant mass spectrum of all true $e^{+} e^{-}$pairs collected during the APEX test run and determine the mass resolution achieved by the experiment. Once the final event sample is produced we can search for the $A^{\prime}$ by performing a peak search on the $e^{+} e^{-}$invariant mass spectrum. The details of the peak search will be discussed in Chapter 8 .

Selecting true $e^{+} e^{-}$pairs requires the ability to distinguish true coincidence events from accidental coincidence events. It also requires sufficient particle identification by the detectors. Only events with good quality tracks in the vertical drift chambers and trajectories falling within the calibrated acceptance of the spectrometers should be used in the final event sample. All aspects of the final event selection will be discussed in this chapter. We will also discuss the procedure for determining the mass resolution of the experiment. Finally, all concerns had by the PAC on the experiment's proposal will be addressed. 


\subsection{Analysis software}

The ADC and TDC data from the detectors is initially stored in a raw CODA format. Each piece of information coming from the electronics is written as a hexidecimal data word. The raw data is converted into ROOT trees using the Hall A ROOT/analyzer facility [29]. The Hall A analyzer is object-oriented code written in $\mathrm{C}++$ and is built on top of the ROOT platform [30]. It provides abstraction for

physical objects such as a spectrometer composed of several detector systems. The analyzer decodes the raw data into physical variables that are ready to be visualized with histograms.

The Hall A analyzer can be installed and used on any computer. However, the APEX test run collected over 500 GB of data, which could not be stored on a single machine. Instead, the raw data is stored on Jefferson Lab's 7 PB storage silo. Each data file requires $\approx 2$ hours on a work-station computer to be analyzed. That means it would take almost three weeks to analyze all of the APEX test run data sequentially on a single computer. Therefore, the analysis of the data was performed on Jefferson Lab's batch farm. The farm's cluster contains $\approx 100$ computing nodes and is capable of running $\approx 1000$ simultaneous jobs, with the limitation of 256 jobs per user at one time [26]. Using the batch farm the analysis of all APEX test run data could be done in just a few days.

\subsection{Particle identification}

When producing the invariant mass spectrum of $e^{+} e^{-}$pairs it is essential to minimize meson contamination of the final event sample. During the test run the meson background was dominated by $\pi^{+}$and $\mu^{+}$. In the Right-HRS, the observed ratio of $e^{+}$to meson background rates, $N_{e^{+}} / N_{\text {meson }}$, is $\sim 1 / 1.5$ with a $2.232 \mathrm{GeV}$ elec- 
tron beam incident on the tantalum foil target (determined using the gas Cherenkov data). Ideally the on-line $N_{e^{+}} / N_{\pi^{+}}$ratio should be $\gtrsim 10 / 1$, so we require an online meson background rejection factor of $\gtrsim 15$ in the Right-HRS. The observed ratio of $e^{-}$to meson background rates in the Left-HRS is $\sim 50 / 1$, so on-line meson background rejection is not required in the Left-HRS.

There are two types of particles identification detectors in the HRSs: the gas Cherenkov counter (GC) and the two-layer lead-glass calorimeter (LG). While the $\mathrm{GC}$ is sensitive to electrons and positrons in the momentum range of interest for this experiment, the probability of pions inducing any signal in the GC is $<2 \%$. This translates to a pion rejection factor of at least 50. The LG has good segmentation and amplitude resolution, which allows a pion rejection factor of at least 100. The simplest trigger configuration which provides on-line $\pi^{+}$rejection uses only the GC in the Right-HRS. This was the configuration used during the APEX test run. The LG was used to analyze the particle identification efficiency of the GC.

Fig. 7.1a shows the ADC sum of all ten PMTs in the Right-HRS GC for the lead target data. The sharp peak at the lower end of the spectrum is assumed to contain the meson background and everything that follows is assumed to contain $e^{+}$. Also shown is the GC ADC spectrum for $e^{+}$(blue) and meson background (red) events as determined by the LG in the Right-HRS. Particles are identified in the LG by plotting the total energy deposited in both layers of the calorimeter over the initial momentum of the incoming particle (Fig. 7.1b). The first peak at the lower end of the spectrum contains $\pi^{+}$, the second peak contains $\mu^{+}$, and the third peak contains $e^{+}$. Also shown are the $e^{+}$(blue) and meson background (red) events as determined by the GC.

The meson background efficiency is calculated by taking the fraction of meson background (as determined by the calorimeter) found in the GC $e^{+}$region. A similar approach can be used to calculate the $e^{+}$detection efficiency of the GC. 


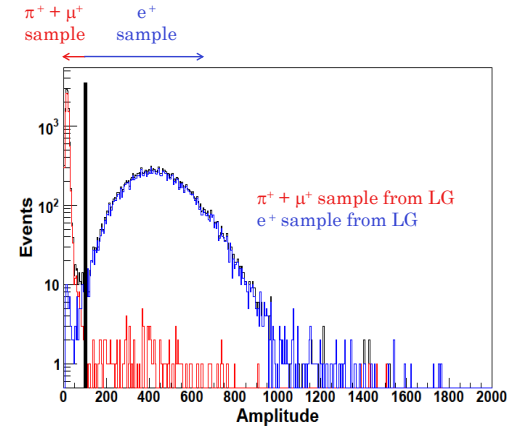

(a)

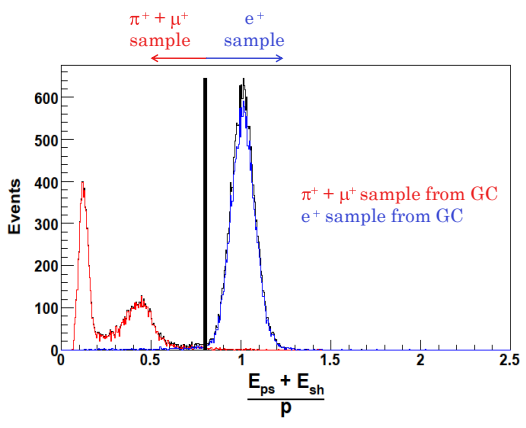

(b)

FIG. 7.1: The lead glass calorimeter was used to study the particle identification efficiency of the gas Chernkov counter used in the coincidence trigger. (A) shows the ADC sum of all ten PMTs in the Cherenkov detector. Events with amplitudes to the right of the black line are considered $e^{+}$, while events with amplitudes to the left are considered meson background. The blue and red lines correspond to $e^{+}$and meson background events as determined by the calorimeter. (B) shows the total energy deposited in both laters of the calorimeter over the initial momentum of the incoming particles. The first peak contains $\pi^{+}$events, the second contains $\mu^{+}$events, and the third contains $e^{+}$events.

When operating at a $\sim 57 \mathrm{kHz}$ Right-HRS trigger rate the gas Cherenkov counter has a $99.5 \% e^{+}$detection efficiency and $98.7 \%$ meson background rejection efficiency. In other words, the meson background is rejected on-line by a factor of $\sim 75$ which is $\sim 5$ times higher than the minimum requirement. Using the GC counters of both HRSs the meson background contamination of the final event sample was determined to be $0.9 \%$, the majority of which comes from $e^{+} \pi^{-}$events.

\subsection{Event selection}

Events accepted into the final data sample were selected by applying a series of cuts to the data. These cuts were designed to select only events with good quality tracks with trajectories residing within the calibrated region of the spectrometers, and to reduce the number of accidental coincidence events. 


\subsubsection{Coincidence timing}

To select $e^{+} e^{-}$coincidence events a triple coincidence trigger was implemented. The trigger consisted of the S2m scintillator planes of both HRSs and the gas Cherenkov counter of the Right-HRS (positron arm). The gas Cherenkov counter was used to provide on-line $\pi^{+}$rejection. Although the trigger was designed to only select $e^{+} e^{-}$pairs there is still a significant amount of background events recorded from accidental coincidence events. Accidentals result from uncorrelated background events that happen to arrive at the detectors within the coincidence timing window.

A timing diagram of the coincidence trigger is shown in Fig. 7.2a. When triggered, the S2m plane of each HRS sends a 20 ns pulse to a coincidence unit, where as width of the gas Cherenkov counter pulse is 10 ns. When all three pulses overlap the data from all detectors is recorded and the event is tagged as a "golden" coincidence event.

The coincidence timing spectrum from a single data run is shown in Fig. $7.2 \mathrm{~b}$. The plot shows the time difference between the T1 trigger (Left-HRS S2m plane) and the T6 trigger ("golden" coincidence trigger). The $\sim 10$ ns wide peak contains true coincidence events, where as the rest of the spectrum contains accidental coincidence events. To select only true coincidence events a 12.5 ns cut was imposed around the true coincidence timing peak (260-272.5 ns).

The rate of accidentals can be estimated from the singles rate in each HRS and the duration of the timing window:

$$
N_{\text {accidentals }} \simeq \sigma N_{\text {Left }} N_{\text {Right }}
$$

where $N_{\text {accidentals }}$ is the rate of accidentals, $N_{\text {Left-HRS }}$ and $N_{\text {Right-HRS }}$ are the singles rates of each HRS, and $\sigma$ is the size of the timing window. This means that $N_{\text {accidentals }}$ scales with the square of the total coincidence trigger rate. The test run data 


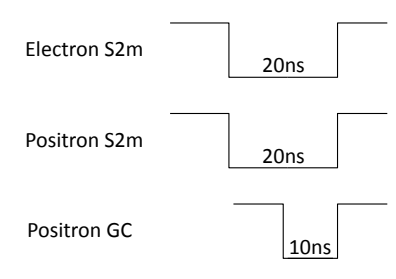

(a)

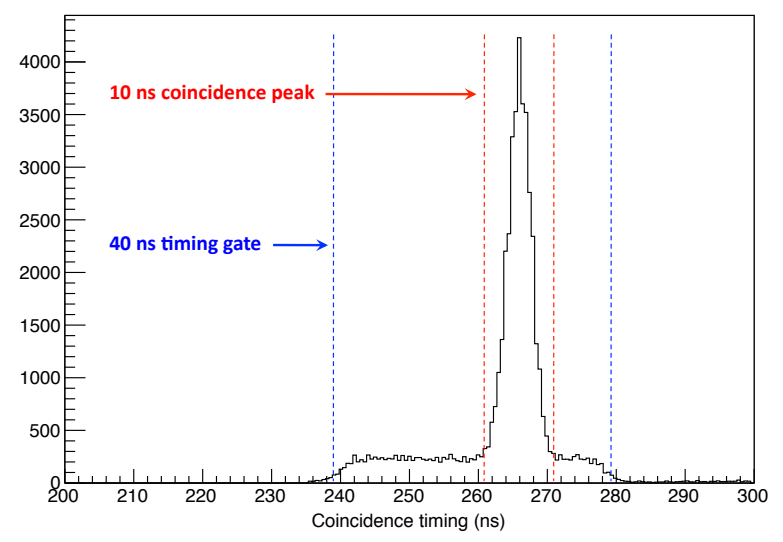

(b)

FIG. 7.2: (A) shows a timing diagram of the coincidence trigger used during the test run. The overlap of both 20 ns S2m pulses and the 10 ns Right-HRS gas Cherenkov pulse creates a "golden" coincidence trigger. (B) shows the time difference between the Left-HRS S2m plane and the coincidence trigger. Notice the $40 \mathrm{~ns}$ duration of the coincidence timing gate and the $10 \mathrm{~ns}$ peak containing true coincidence events.

had a signal to background ratio of $\sim 5 / 1$ (Fig. 7.2b). The full experiment will use a multi-foil target with a total target thickness of $4.3 \%$ radiation lengths (see Sec. 4.4.1), which is $\sim 10$ times thicker than the test run target. Also, the PREX collimators described in Sec. 7.3.2 will not be present during the full experiment, so the spectrometer acceptance will be increased by a factor of $\sim 2$. The resulting signal to background ratio will be $\sim 1 / 4$, however, this can be improved offline to $\sim 12 / 1$ by applying timing cuts (factor of $\sim 5$ accidental reduction) and correcting on multiple target hits (factor of $\sim 10$ accidental reduction).

\subsubsection{Acceptance cuts}

To accurately reconstruct the momentum of particles at the target it's important to avoid recording events with trajetories outside the calibrated region of the 


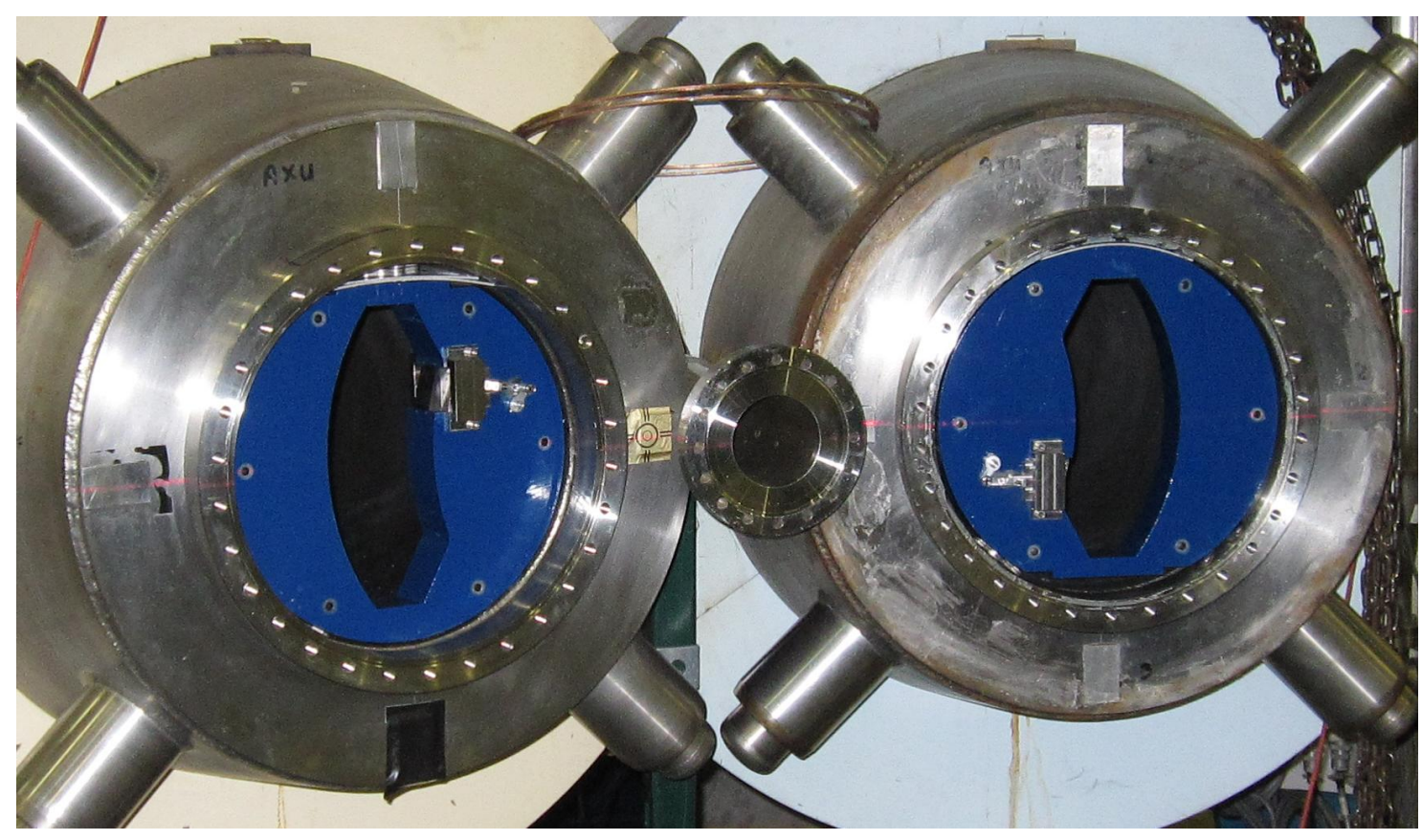

FIG. 7.3: The presence of collimators from the PREX experiment reduced the solid angle acceptance of each spectrometer from the nominal $4.1 \mathrm{msr}$ to $\simeq 2.8(2.9) \mathrm{msr}$ for the left (right) HRS.

spectrometers. The PREX experiment [24], which took place in Hall A prior to the APEX test run, had installed collimators at the entrance of each spectrometer (Fig. 7.3). Due to a lack of time the collimators were unable to be removed for the test run, and therefore restricted the acceptance of the HRSs.

For each spectrometer, the acceptance is defined by three sets of two-dimensional polygonal cuts made in $\theta_{t g}$ vs. $\phi_{t g}, \delta_{t g}$ vs. $\phi_{t g}$, and $\delta_{t g}$ vs. $\theta_{t g}$. The $\theta_{t g}, \phi_{t g}$, and $\delta_{t g}$ variables correspond to the vertical angle, horizontal angle, and momentum deviation respectively in the target coordinate system as described in Sec. 6.3.1. The cuts are shown in Fig. 7.4. The solid angle acceptance after applying these cuts is $\simeq 2.8(2.9) \mathrm{msr}$ for the left (right) HRS, where as the nominal solid angle acceptance 

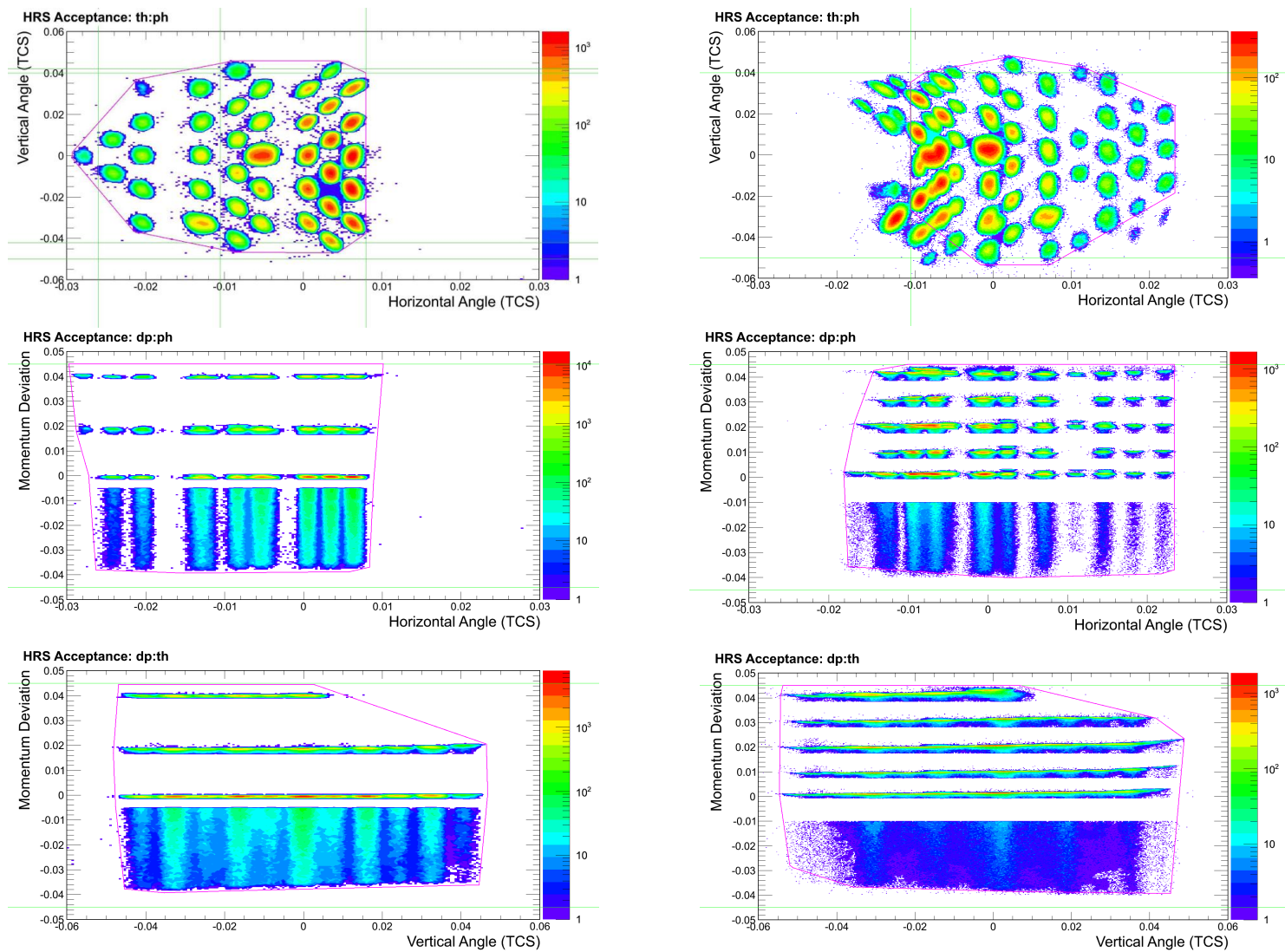

FIG. 7.4: The acceptance of each spectrometer is defined by 2-D graphical cuts applied to $\theta_{t g}$ vs. $\phi_{t g}, \delta_{t g}$ vs. $\phi_{t g}$, and $\delta_{t g}$ vs. $\theta_{t g}$. The cuts are shown by the area outlined in pink.

without the presence of the PREX collimators is $4.3 \mathrm{msr}$.

\subsubsection{Final event sample}

The timing and graphical cuts described above were applied to the data to select the final event sample. Using the timing distribution in Fig. $7.2 \mathrm{~b}$ one finds that $14.9 \%$ of events in the coincidence peak contain accidentals. An easy way to reduce the accidentals by a factor $\sim 2$ is to demand the sum of the $e^{-}$and $e^{+}$ momentum be less than the beam energy. After applying this energy cut the fraction of accidentals in the coincidence peak reduced to $7.4 \%$, as expected.

In addition to the cuts described above we require good quality track recon- 
struction in the VDC. If the analyzer is unable to reconstruct a good quality track for an event, e.g. there is ambiguity between clusters in the $\mathrm{U}$ and $\mathrm{V}$ planes of a VDC (see Sec. 7.4), it will set the root variable tr.ok to zero, otherwise the variable will be set to one. Events with good quality tracks are selected by requiring that tr.ok $==1$. Multiple tracks observed in the same spectrometer for a single event create ambiguity in the track selection. Therefore, we require that events have only one observed track in each spectrometer.

After applying all cuts to the data the final event sample contains 770,500 events with only $0.9 \%$ contamination from meson backgrounds, and $7.4 \%$ accidental $e^{+} e^{-}$coincidence events.

\subsection{Track reconstruction}

The momentum reconstruction of $e^{+} e^{-}$pairs begins with the measurement of the position and angle of the incoming particles in the focal plane. This measurement is made using the VDCs, the details of which are described in Sec. 3.2.3. Tracks enter the VDCs nominally at $45^{\circ}$ and produce signals in 3-7 wires at a time. TDCs measure the time interval between the wire signals and a common stop signal that is generated from one of the triggers.

Activated wires adjacent to one another are organized into clusters. The drift time, or the time it takes ionized electrons to travel from the trajectory to the sense wires, is determined for each wire within the cluster. Wires at the edge of a cluster have a longer drift time than wires near the center of the cluster, as illustrated in Fig. 7.5. The cluster search algorithm scans the VDC data for 'V' shaped clusters in time. The drift time of each wire is measured by the TDCs and converted into the drift distance, or the perpendicular distance from the trajectory to the wire plane. A linear fit is performed on the drift distances within a cluster, giving the 

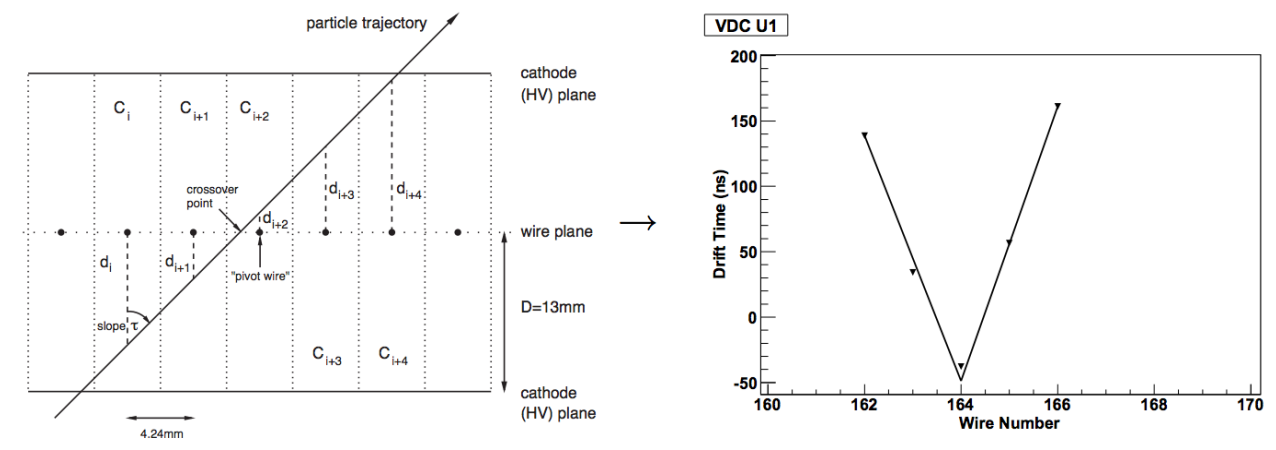

FIG. 7.5: Example of a VDC cluster containing five activated wires. Wires toward the edge of the cluster have a longer drift time than wires near the center. Thus, the drift times of the wires within the cluster exhibit a ' $V$ ' shape.

approximate track angle, $\theta_{Q_{i}}$, and intersection point, $Q_{i}$, at the wire plane [31].

Two VDCs are used in each HRS, each with $\mathrm{U}$ and $\mathrm{V}$ planes. As shown in Fig. 7.6a, the global trajectory angles are defined according to

$$
\tan \theta_{Q}=\frac{Q 2-Q 1}{d}
$$

where $Q \ni(U, V)$ and the distance $d$ between like wire planes (U1 and U2; V1 and $\mathrm{V} 2)$ is $\simeq 0.335 \mathrm{~m}$ [5]. Keep in mind that the wires in the $\mathrm{U}$ planes are orthogonal to those in the $\mathrm{V}$ planes. The global angles $\theta_{Q}$ can then be used to project the track coordinate measured by the V1 plane into the U1 plane, as shown in Fig. $7.6 \mathrm{~b}$. The 


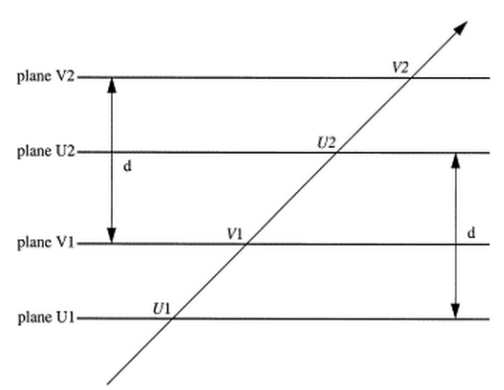

(a)

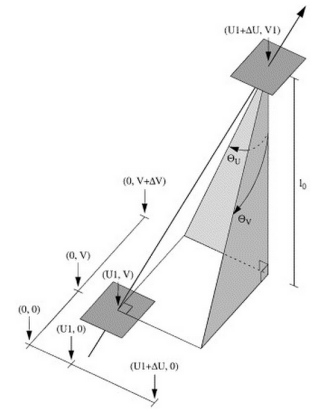

(b)

FIG. 7.6: (A) The intersection points at like planes (U1 and U2; V1 and V2) can be used to determine the global trajectory angles, $\theta_{U}$ and $\theta_{V}$. Note that the wires in the $\mathrm{U}$ planes are orthogonal to those in the $\mathrm{V}$ planes. The wires in both planes are oriented $45^{\circ}$ with respect to the plane of the page. (B) The global trajectory angles can then be used to project the V1 plane coordinates into the U1 plane.

variable $\mathrm{V}$ is the $\mathrm{V} 1$ coordinate projected into the $\mathrm{U} 1$ plane, and is given by

$$
V=V 1-\Delta V=V 1-l_{0} \tan \theta_{V}
$$

where the distance $l_{0}$ between the $\mathrm{U} 1$ and V1 planes is $26 \mathrm{~mm}$ [5]. The design of this two-VDC system allows all trajectories to be characterized by a set of four unique coordinates $\left(\mathrm{U}, \mathrm{V}, \theta_{U}\right.$, and $\left.\theta_{V}\right)$. These coordinates can then be transformed into other coordinate systems, as described in Sec. 6.3.

A couple of modifications were made to the track reconstruction algorithm for the analysis of the test run data. The track reconstruction required that the time difference between the $\mathrm{U}$ cluster and $\mathrm{V}$ cluster be within $\pm 40 \mathrm{~ns}$. Also, if there is any ambiguity in the association of $\mathrm{U}$ clusters with $\mathrm{V}$ clusters, i.e. a cluster in the $\mathrm{U}$ plane is associated with more than one $\mathrm{V}$ cluster, then the tracking ends and the event is flagged. Fig. 7.7 demonstrates both of these modifications.

An understanding of the track reconstruction efficiency is required to compute the rate of $e^{+} e^{-}$pair production during the test run. The track reconstruction 


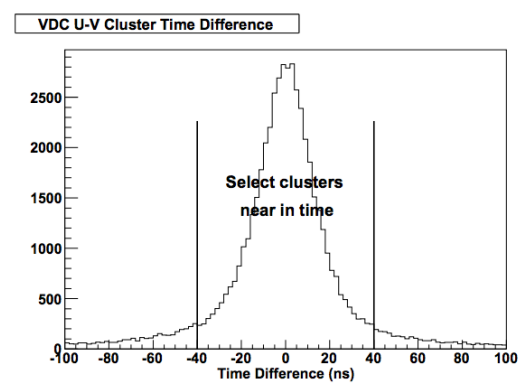

(a)

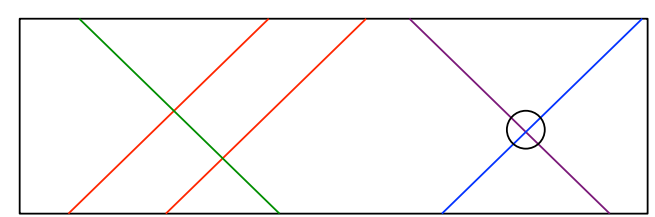

(b)

FIG. 7.7: Modifications made to the tracking algorithm. (A) shows the distribution of the time difference between the $\mathrm{U}$ and $\mathrm{V}$ clusters. A cut was made requiring that the time difference be within $\pm 40 \mathrm{~ns}$. (B) demonstrates a case where a U cluster (green line) is associated with more than one $\mathrm{V}$ clusters (red lines). Tracks are not constructed for events exhibiting UV association ambiguity.

TABLE 7.1: "Loose" acceptance cuts used for estimating the track reconstruction efficiency. All variables are in the detector coordinate system.

\begin{tabular}{cc} 
Left-HRS & Right-HRS \\
\hline$-0.7 \mathrm{~m}<x<0.6 \mathrm{~m}$ & $-0.7 \mathrm{~m}<x<0.6 \mathrm{~m}$ \\
$-0.05 \mathrm{~m}<y<0.03 \mathrm{~m}$ & $-0.04 \mathrm{~m}<y<0.04 \mathrm{~m}$ \\
$-150 \mathrm{mrad}<\theta<120 \mathrm{mrad}$ & $-150 \mathrm{mrad}<\theta<120 \mathrm{mrad}$ \\
$-25 \mathrm{mrad}<\phi<25 \mathrm{mrad}$ & $-20 \mathrm{mrad}<\phi<25 \mathrm{mrad}$ \\
\hline
\end{tabular}

efficiency can be estimated by determining how often the reconstructed trajectories of true $e^{+} e^{-}$pair events fall within the active region of the VDCs. True coincidence events are selected by applying the coincidence peak timing cut described above. The number of these events with trajectories passing through the acceptable region of the VDC plane is determined by applying the "loose" acceptance cuts defined in Table 7.1. The cuts are made on the detector coordinate system variables (see Sec. 6.3.1) and are shown in Fig. 7.8. The track reconstruction efficiencies are calculated by taking the ratio of coincidence events that pass the cuts to the total coincidence events, $N_{\text {cut }} / N$. The track reconstruction efficiencies for the LHRS (RHRS) are $99.0 \%(98.2 \%)$. 

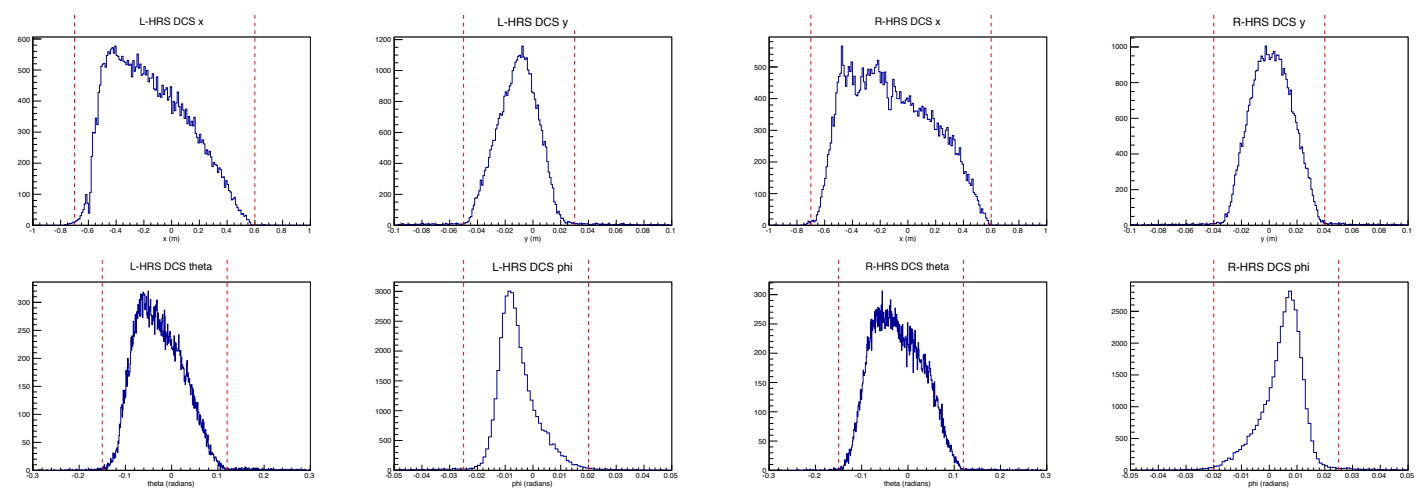

(a)

(b)

FIG. 7.8: Plots illustrating the "loose" acceptance cuts used to determine the track reconstruction efficiency for the (A) Left-HRS and (B) Right-HRS. All variables are in the detector coordinate system.

\subsection{Invariant mass calculation and comparison to}

\section{MC data}

The invariant mass of $e^{+} e^{-}$pairs can be calculated as

$$
m_{0}^{2}=\left(E_{e^{+}}+E_{e^{-}}\right)^{2}-\left(\vec{p}_{e^{+}}+\vec{p}_{e^{-}}\right)^{2}
$$

Resolving the parentheses:

$$
m_{0}^{2}=E_{e^{+}}^{2}+E_{e^{-}}^{2}+2 \cdot E_{e^{+}} \cdot E_{e^{-}}-\vec{p}_{e^{+}}^{2}-\vec{p}_{e^{-}}^{2}-2 \cdot \vec{p}_{e^{+}} \cdot \vec{p}_{e^{-}}
$$

Substituting in $m_{e^{ \pm}}^{2}=E_{e^{ \pm}}^{2}-\vec{p}_{e^{ \pm}}^{2}$ gives

$$
m_{0}^{2}=m_{e^{+}}^{2}+m_{e^{-}}^{2}+2 \cdot E_{e^{+}} \cdot E_{e^{-}}-2 \cdot \vec{p}_{e^{+}} \cdot \vec{p}_{e^{-}} .
$$

Since the masses of $e^{+}$and $e^{-}$are negligible, the invariant mass can be expressed

$$
m_{0}^{2}=2 \cdot\left|p_{e^{+}}\right|\left|p_{e^{-}}\right|-2 \cdot \vec{p}_{e^{+}} \cdot \vec{p}_{e^{-}} .
$$


Eq. 7.7 can be written in terms of the $x, y$, and $z$ momentum components in the lab frame or "Hall A Coordinate System" (HCS):

$$
m_{0}^{2}=2 \cdot\left(\left|p^{e^{+}}\right|\left|p^{e^{-}}\right|-\left(p_{x}^{e^{+}} p_{x}^{e^{-}}+p_{y}^{e^{+}} p_{y}^{e^{-}}+p_{z}^{e^{+}} p_{z}^{e^{-}}\right)\right)
$$

In the $\mathrm{HCS}+z$ points in the beam direction and $+y$ points upward.

Each HRS measures the $\delta, \theta$, and $\phi$ variables in the "Target Coordinate System" or TCS, whose z-axis points towards the center of HRS's entrance. $\delta=1-\frac{p}{p_{0}}$, where $p_{0}$ is the central momentum setting of the HRS, and $\theta$ and $\phi$ are the vertical and horizontal angles with respect to $z_{\text {TCS }}$. These variables can be translated into the three dimensional momentum variables of the particle at the vertex in the HCS using the equations

$$
\begin{gathered}
p_{x}=p_{z_{\mathrm{TCS}}}\left(\tan \phi \cos \theta_{0}+\sin \theta_{0}\right) \\
p_{y}=p_{z_{\mathrm{TCS}}} \tan \theta \\
p_{z}=p_{z_{\mathrm{TCS}}}\left(\cos \theta_{0}-\tan \phi \sin \theta_{0}\right)
\end{gathered}
$$

where

$$
p_{z_{\mathrm{TCS}}}=\frac{p_{0}(1+\delta)}{\sqrt{1+\tan \theta^{2}+\tan \phi^{2}}}
$$

and $\theta_{0}$ is the angle of the HRS with respect to the beam $\left(+5^{\circ}\right.$ for the Left-HRS and $-5^{\circ}$ for the Right-HRS).

After making the final event selection of the entire production data sample, the invariant mass of each event was calculated. Over 770,500 true $e^{+} e^{-}$pairs were collected in the mass range of 175-250 MeV. Fig. 7.9 shows the invariant mass spectrum for all good $e^{+} e^{-}$pairs collected during the test run. The bin width of the histogram is $50 \mathrm{keV}$.

The production of leading order QED trident processes was calculated using 


\section{APEX Test Run Data, $\sim 770 \mathrm{~K}$ evts}

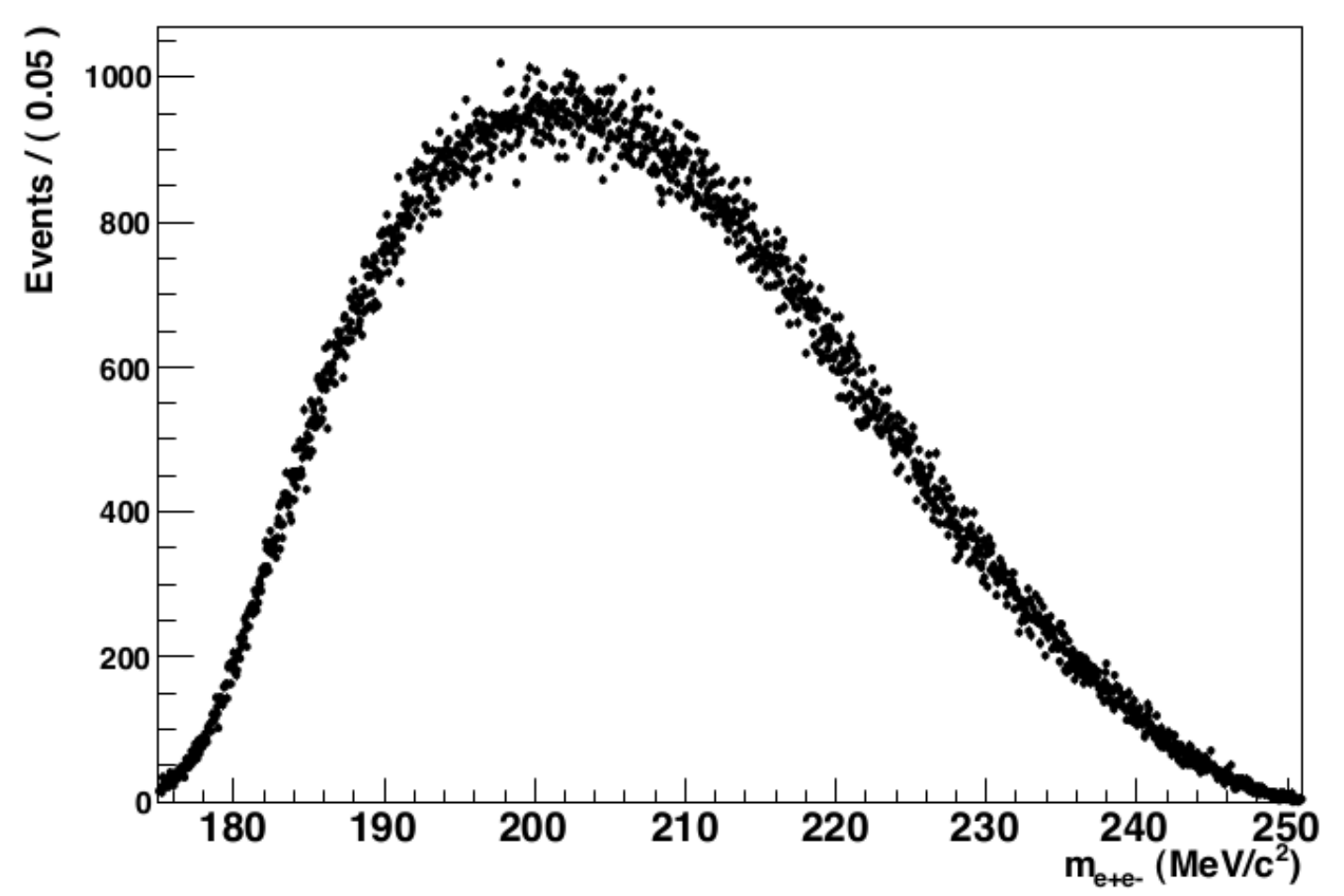

FIG. 7.9: The invariant mass spectrum of all true $e^{+} e^{-}$pairs collected during the test run. Data was collected in the mass range of $175-250 \mathrm{MeV}$.

MadGraph and MadEvent, as discussed in Sec. 4.2. The acceptance cuts that were applied to the final data sample of the test run (Sec. 7.3.2) were also applied to the calculated coincident event sample. The invariant mass spectrum of the calculated event sample overall normalized to the test run data is shown in Fig. 7.10. The calculated QED trident spectrum was added to the accidental event sample, which consisted of trigger events residing outside of the coincidence timing peak (see Fig. 7.2b). The QED trident rates calculated for the test run configuration agree within a few percent with the actual data. The differential momentum and angular distributions agree with the data to within $5-10 \%$. Fig. 7.11 compares the Monte Carlo data (histograms) with the test run data (black dots). 


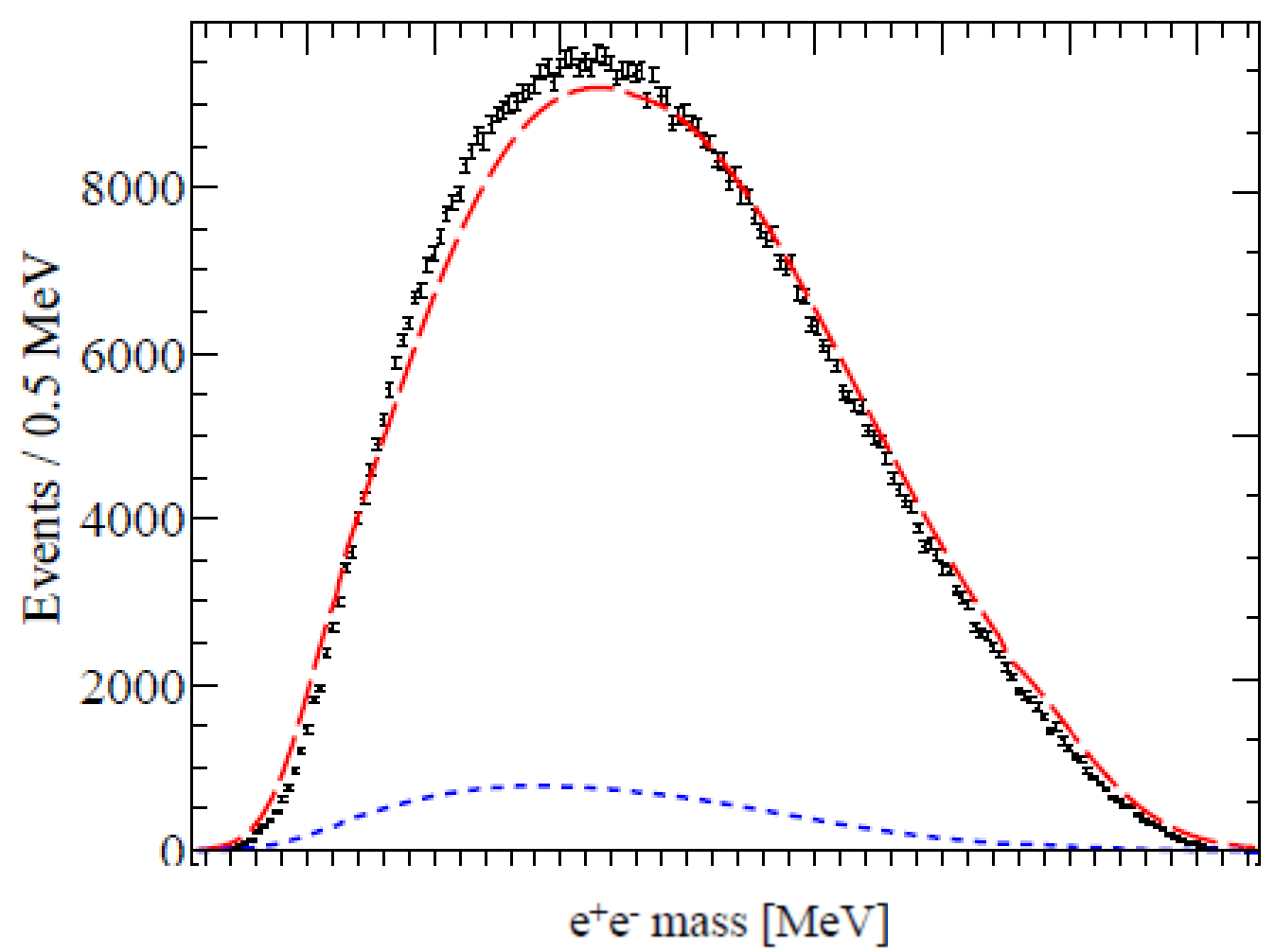

FIG. 7.10: The invariant mass spectrum of $e^{+} e^{-}$pair events collected during the test run (black points, with error bars), accidental coincidence events (blue short-dash line), and the QED calculation of trident background added to the accidental event sample (red long-dash line).

\subsection{Mass resolution}

The $A^{\prime}$ will appear as a small peak on top of a QED trident background, with the width of the peak corresponding to the mass resolution of the experiment. Therefore, determining the mass resolution is essential to understanding the sensitivity of the peak search. The invariant mass of $e^{+} e^{-}$pairs can be calculated to leading order according to

$$
m^{2} \approx p_{0}^{2}\left(4 \theta_{0}^{2}+4 \theta_{0}^{2} \delta_{+}+4 \theta_{0}^{2} \delta_{-}+8 \theta_{0}\left(\phi_{+}-\phi_{-}\right)+2 \theta_{+} \theta_{-}\right)
$$



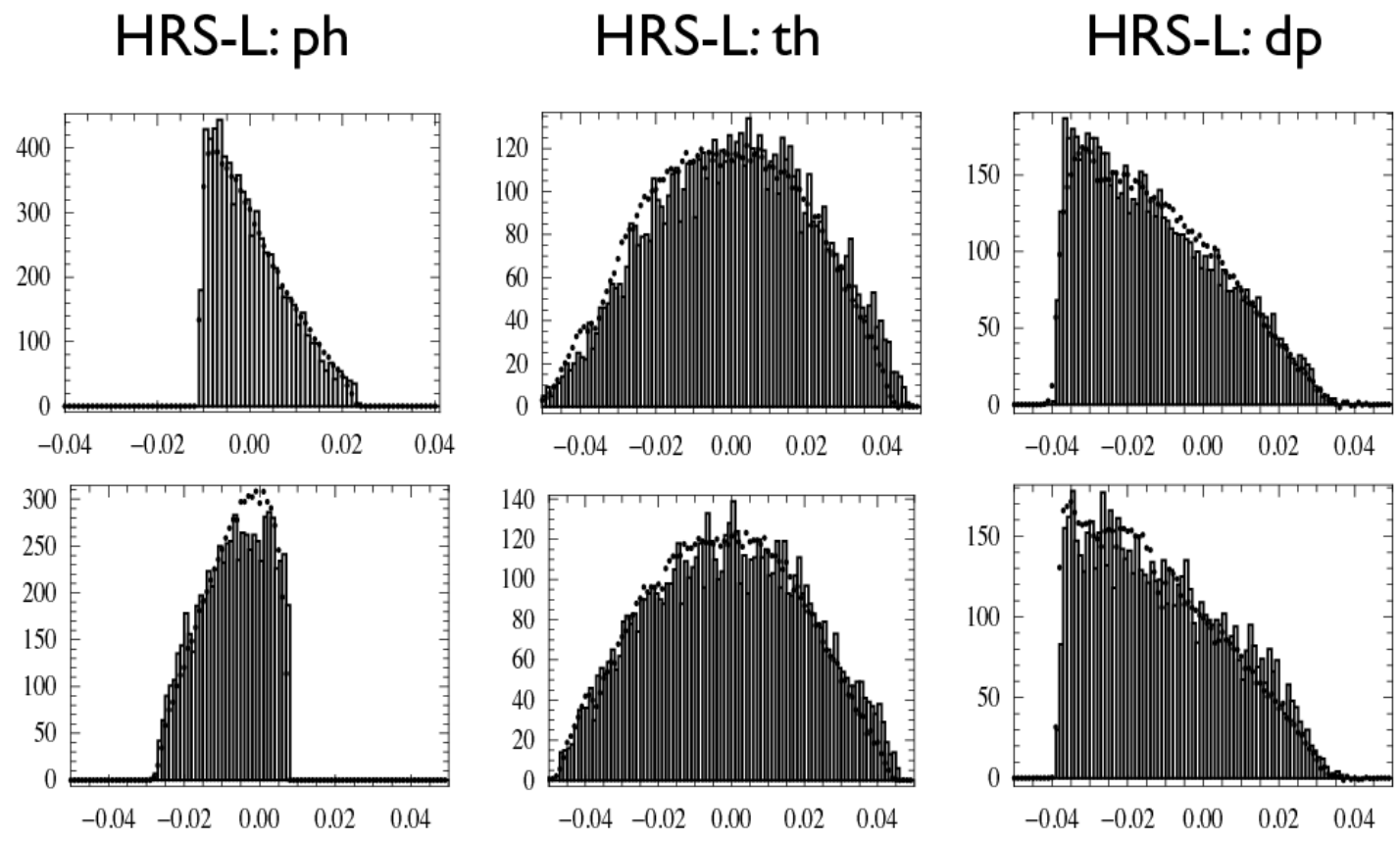

HRS-R: ph
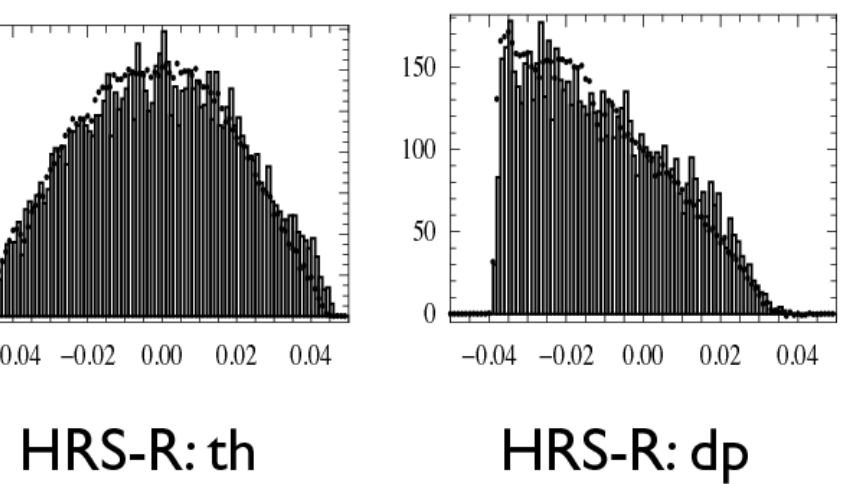

\section{HRS-R: dp}

FIG. 7.11: Angular distributions of the Monte Carlo simulations (histogram) and the test run data (black dots).

where $p_{0}$ is the central momentum setting of both HRSs, $\theta_{0}$ is the angle between the beamline and the central trajectory of the spectrometer, $\delta_{ \pm}$is the relative momentum, $\phi_{ \pm}$is the horizontal angle, $\theta_{ \pm}$is the vertical angle, and all variables are in the target coordinate system. Due to the excellent HRS relative momentum resolution of $\varnothing\left(10^{-4}\right)$, the mass resolution is completely dominated by horizontal and vertical angular resolutions.

\subsubsection{Angular resolution}

The angular resolution can be broken up into 3 separate parts: multiple scattering inside of the target, track measurement errors by the HRS detectors, and imperfections in the magnetic optics reconstruction matrix. We will discuss the 
calculation of all three contributions and how each affects the final mass resolution.

\section{Multiple scattering in the target}

When traveling through a medium electrons and positrons experience many small angle deflections due to Coulumb scattering from nuclei. The resulting angular distribution from this multiple scattering is given by / citepmultiscat

$$
\Omega=\frac{13.6 \mathrm{MeV}}{p} \sqrt{\frac{X}{X_{0}}}\left(1+0.038 \ln \frac{X}{X_{0}}\right)
$$

where $p$ is the particle momentum, $x$ is the target thickness, and $x_{0}$ is the radiation length of the target material. The tantalum foil target used during the test run has a thickness of $22 \mathrm{mg} / \mathrm{cm}^{2}$, or $0.0032 X_{0}$. This target thickness leads to a $0.37 \mathrm{mrad}$ contribution to the uncertainty in both horizontal and vertical angles.

\section{Track measurement errors and imperfections in the optics matrix}

To calibrate the magnetic optics matrix elements, data was taken with a tungsten sieve plate inserted at the entrance of each spectrometer, as discussed in Sec. 6.3.3. The sieve plates each have a grid of holes drilled through them with known positions and widths. When electrons scatter from the target toward the spectrometer only electrons with trajectories passing directly through the sieve holes will be detected. Therefore the reconstructed trajectories of the electrons projected at the sieve plane should resemble the sieve hole pattern. The reconstruction of the holes can be compared with the surveyed locations and widths of the holes to

determine the track measurement errors of the HRS and the imperfections in the final optics reconstruction matrix.

The $\mathrm{x}$ and $\mathrm{y}$ distributions of each hole were plotted and fit with a guassian on top of a linear background (Fig. 7.12). The reconstructed positions and widths of each 
hole are extracted from the fit and compared with the surveyed hole positions and widths. The track measurement uncertainty of the HRS detectors was determined by comparing the reconstructed and surveyed hole widths:

$$
\Delta_{\sigma_{x}}^{2}=\sigma_{x_{\text {reconstructed }}}^{2}-\sigma_{x_{\text {surveyed }}}^{2}, \quad \Delta_{\sigma_{y}}^{2}=\sigma_{y_{\text {reconstructed }}}^{2}-\sigma_{y_{\text {surveyed }}}^{2}
$$

The imperfections of the optics reconstruction matrix were determined by comparing the reconstructed and surveyed hole positions:

$$
\Delta_{x}=x_{\text {reconstructed }}-x_{\text {surveyed }}, \quad \Delta_{y}=y_{\text {reconstructed }}-y_{\text {surveyed }} .
$$

These results were converted into angular uncertainties by dividing them by the distance to the center of the target and taking the inverse tangent:

$$
\phi=\arctan \frac{x}{D_{\text {target }}}
$$

Table 7.2 summarizes the contributions to the angular resolution averaged over all sieve holes located within the calibrated acceptance.

TABLE 7.2: Summary of the contributions to the angular resolution averaged over all sieve holes located within the calibrated acceptance.

\begin{tabular}{|l|cc|cc|}
\hline & \multicolumn{2}{|c|}{ Left-HRS } & \multicolumn{2}{c|}{ Right-HRS } \\
\hline & $\begin{array}{c}\text { Track } \\
\text { reconst. } \\
\text { errors }\end{array}$ & $\begin{array}{c}\text { Optics matrix } \\
\text { imperf. }\end{array}$ & $\begin{array}{c}\text { Track } \\
\text { reconst. } \\
\text { errors }\end{array}$ & $\begin{array}{c}\text { Optics matrix } \\
\text { imperf. }\end{array}$ \\
\hline Horizontal $(\mathrm{mrad})$ & 0.33 & 0.1 & 0.43 & 0.1 \\
Vertical $(\mathrm{mrad})$ & 1.85 & 0.22 & 1.77 & 0.22 \\
\hline
\end{tabular}



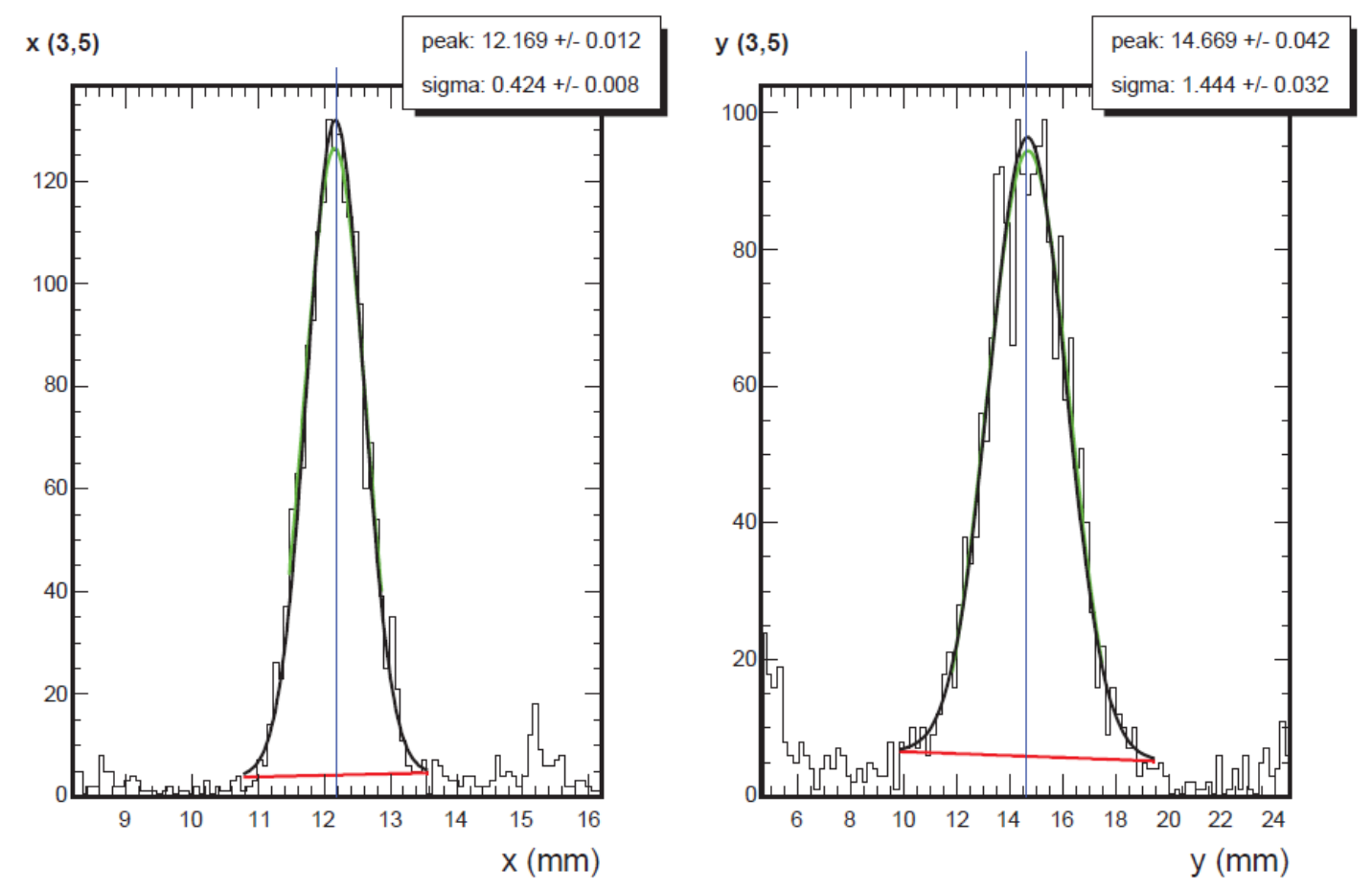

FIG. 7.12: The $\mathrm{x}$ and $\mathrm{y}$ distributions for a single sieve hole. The distributions were fit with a guassian (black) on top of a linear background (red). The blue line indicates the surveyed location of the hole. The mean and the sigma of the fit correspond to the reconstructed position and width of the hole.

\subsubsection{Determining the mass resolution}

In this section we will discuss the procedure used to determine the mass resolution of the APEX test run. The first step is to simulate a new invariant mass spectrum by adjusting existing data according to the angular resolutions of the experiment. The simulation was done using real events from a single production run. For each event the horizontal and vertical components of the trajectories were randomly adjusted. More specifically, a new angle was randomly selected according to a Gaussian distribution centered at the real angle for that event and with a $\sigma$ equal to the angular resolution (Fig. ??). The angular resolution was determined for each 
sieve hole individually, so the angular resolution associated with the hole located closest to the particle's trajectory was used in generating the new angle (multiple scattering was also included). After the new angles were generated a new invariant mass was calculated for that event.

After simulating new invariant masses for all events, the difference between the fake mass and real mass was plotted in a histogram (Fig. 7.13). The RMS value of the resulting distribution corresponds to the mass resolution of the experiment. Finally, the mass resolution was determined for different values of invariant mass. Fig. ?? shows how the mass resolution varies with invariant mass. The resulting fit function is

$$
-0.0001 x^{2}+0.058 x-5.3961
$$

where $x$ is the invariant mass. This equation was used in the peak search, the details of which are discussed in Ch. 8.

\subsection{Addressing the PAC concerns}

The purpose of the APEX test run was to address several concerns had by the PAC35 about the experiment's proposal. In this section we will present the results of our investigation of the issues raised by the PAC35 report. These results were also discussed at the "Searching for a New Gauge Boson at JLab" workshop [32.

\section{The multi-foil target}

The multi-foil target has a superior design over the wire-mesh target presented in the original proposal. The details of the multi-foil target are discussed in Sec. 4.4.1. The target was constructed and shipped to Jefferson Lab, although it was never installed due to time constraints. 


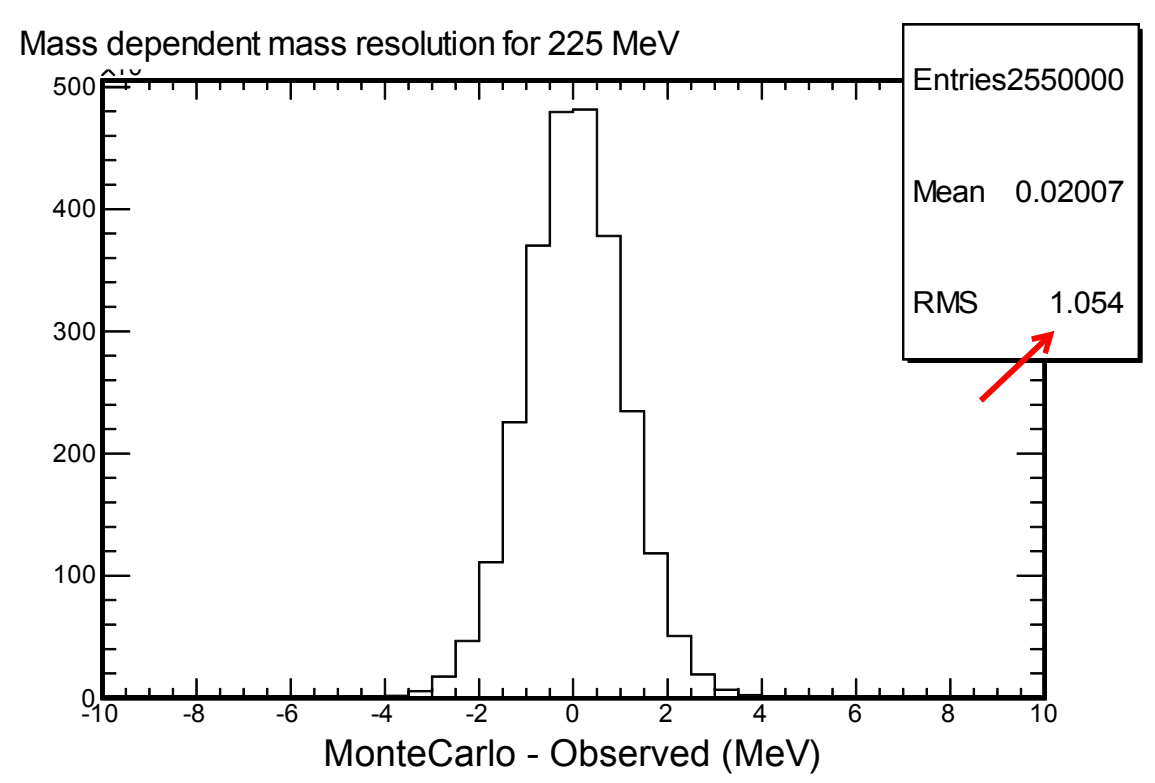

FIG. 7.13: New invariant masses were simulated by adjusting real data to the angular resolutions of the experiment. The differences between the simulated masses and real masses are shown in the histogram. The $\sigma$ of this distribution corresponds to the mass resolution of the experiment.

The vertex resolution achieved during the Pentaquark experiment was $1.1 \mathrm{~cm}$ [33]. This resolution would be sufficient for resolving the production target foils which are separated by $5.5 \mathrm{~cm}$ along the beam line.

\section{Central scattering angle}

Fine resolution of the $e^{+} e^{-}$invariant mass is required to achieve a high precision search for the $A^{\prime}$. The resolution of the invariant mass measurements is dominated by the horizontal angular resolution which has contributions coming from multiple scattering in the target, uncertainties in the track reconstruction by the HRS detectors, and imperfections in the optics reconstruction matrix. Multiple scattering in the target contributes $\sim 0.4 / p[\mathrm{GeV}]$ mrad in each HRS with a spectrometer setting of $p$, contributing $\sim 0.5 \mathrm{MeV}$ to the mass resolution. The second and third contri- 


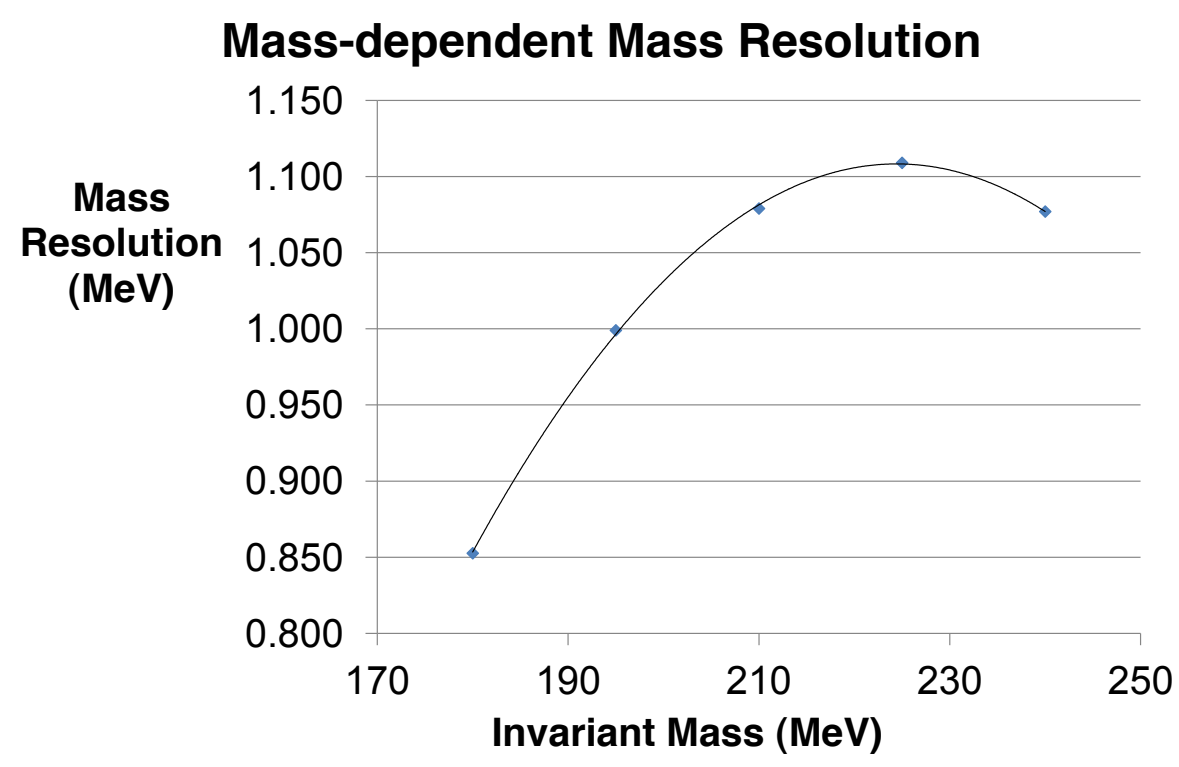

FIG. 7.14: The mass resolution as a function of invariant mass. The resulting fit function was used in the final peak search.

butions combined give an uncertainty of $\sim 0.5 \mathrm{mrad}$ in each HRS, and contributes $0.7 \mathrm{MeV} \dot{p}[\mathrm{GeV}]$ to the mass resolution.

Due to the high momentum resolution of the HRS, particle momenta are measured to within $10^{-4}$. A fractional momentum resolution of $5 \times 10^{-4}$ only contributes $0.07 \mathrm{MeV} \dot{p}[\mathrm{GeV}]$ to the mass resolution, which is considered negligible. The $1 \mathrm{mrad}$ uncertainty in vertical angles also only contributes $0.12 \mathrm{MeV} \dot{p}[\mathrm{GeV}]$ to the mass resolution.

The position of the spectrometers defines the absolute scale of the $e^{+} e^{-}$pair invariant mass, but has a small effect on the event-by-event mass resolution. A Monte Carlo simulation was done to determine what effect the uncertainty in the angle between the spectrometers would have on the mass resolution. The simulation demonstrated that a $1 \mathrm{mrad}$ uncertainty would only contribute $8 \times 10^{-5}$ to the eventby-event invariant mass resolution. 


\section{VDC operation at high rates}

For the proposed experiment the VDCs must be capable of operating at a maximum rate of $5 \mathrm{MHz}$. Such high rate operation was made possible by utilizing the the custom amplifier/discriminator cards described in Sec. 4.4.3. These A/D cards allow the VDCs to operate at $-3.5 \mathrm{kV}$ instead of $-4 \mathrm{kV}$. Operating at a reduced voltage allows long term operation while still using the standard VDC gas mixture. The custom A/D cards were installed during the test run and data was taken using a lead target in order to achieve tracking rates up to $5 \mathrm{MHz}$.

The VDC wire efficiency during high rate data taking was checked during the test run. For every event, all wires in a given VDC plane are scanned. Events are identified where two wires in the plane fire and a third wire is between them. If the third wire also fires, the event is defined to be efficient. An example of the VDC wire efficiency of a single VDC plane for high rate data is shown in Fig. 7.15 . The drift time distribution during high rate data taking exhibited a normal profile, as shown in Fig. 7.16. Also, the drift time to coordinate calibration is the same for low and high rate data, as shown in Fig. 7.17. We observe a small reduction in cluster size at high rates (Fig. 7.18), which is due to reduced efficiency. Finally, the test run demonstrated a track reconstruction efficiency of $\sim 60 \%$ for high rate operation.

\section{$\pi^{+}$Rejection at high rates}

The Right-HRS GC detector is used in the trigger to provide on-line $\pi^{+}$rejection. To study the GC pion rejection efficiency at high rates, data was taken with a $30 \mu \mathrm{A}$ electron beam incident on the lead target described in Sec. 5.2.1. The Right-HRS trigger rate for this data was $\sim 765 \mathrm{kHz}(85 \mathrm{kHz}$ positrons and $680 \mathrm{kHz}$ meson background). Fig. 7.19 shows the GC amplitude spectrum for the high rate data. Just as before, the sharp peak on the left contains meson background whereas 


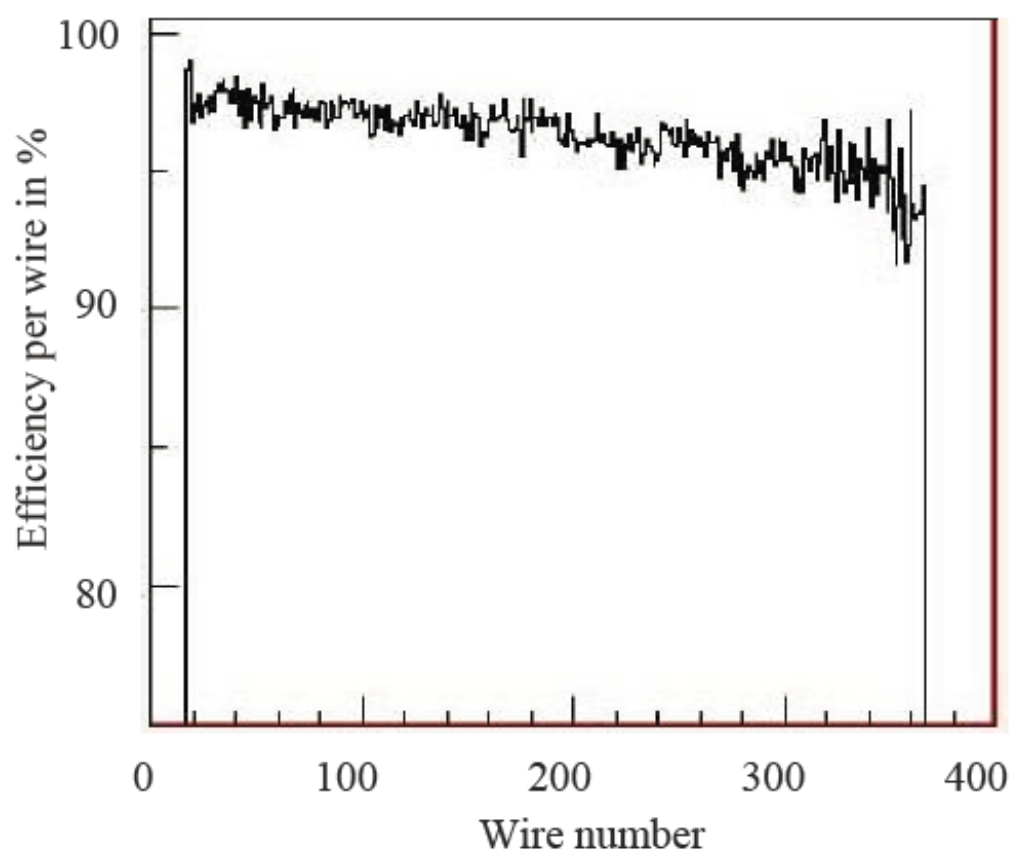

FIG. 7.15: The VDC wire efficiency for a single plane for high rate data. Events are identified where two wires fire and a third wire is between them. If the third wire also fires, the event is considered efficient. Otherwise the event is defined as inefficient.

the distribution that follows contains positrons. The red and blue distributions correspond to the meson background and $e^{+}$samples respectively as determined by the LG. The pion rejection efficiency of the GC was determined using the same method described in Sec. ??. Using this analysis we obtain an on-line meson background rejection factor of 30 for data taken at close to the expected maximum rate.

\section{Coincidence timing window}

For high rate data taking it is important to have a small coincidence timing window in order to minimize the DAQ deadtime and avoid recording excess accidental coincidence events. The APEX test run used a trigger consisting of a coincidence between the two S2m scintillator planes and the GC of the positron arm (Sec. 5.3). Both S2m planes produced 20 ns signals while the GC produced 10 ns signals. The 


\section{VDC Drift Time Spectrum}

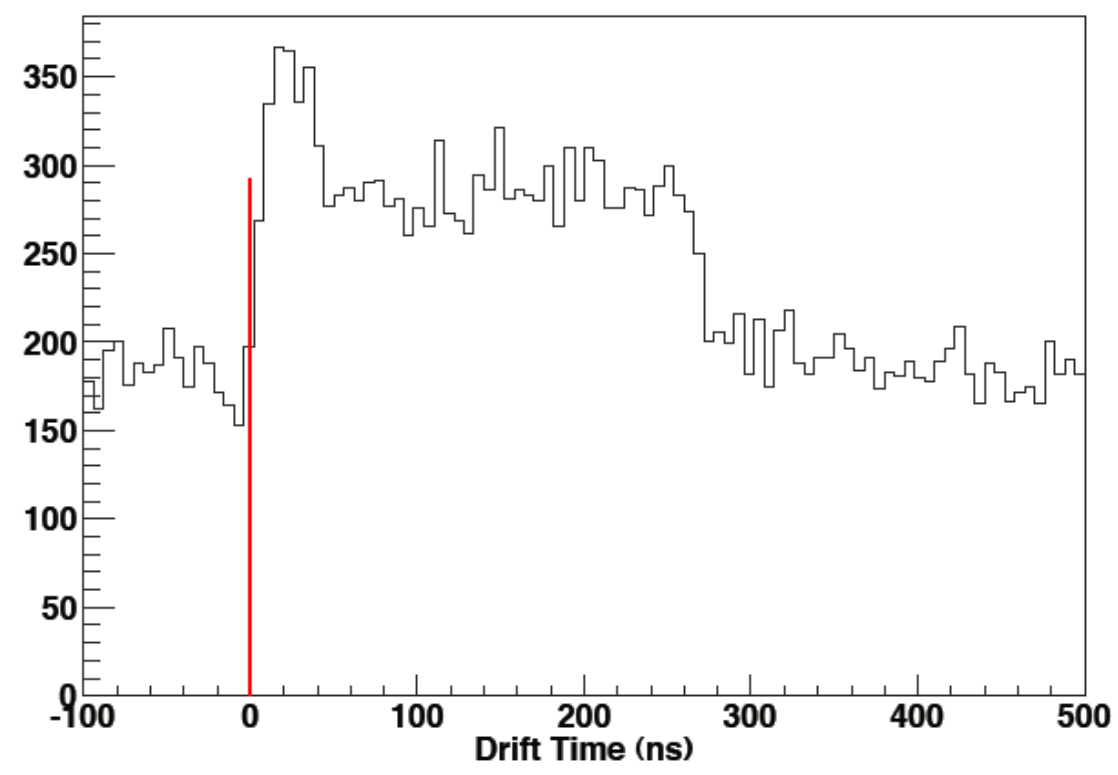

FIG. 7.16: An example of the VDC drift time during high rate operation.

overlap of these three signals provides a coincidence timing window of 40 ns. The S0 counters of each arm were used to align the average timing of the S2m and GC signals. This fine tuning allowed us to produce a $15 \mathrm{~ns}$ wide timing peak consisting of true coincidence events, as shown in Fig. $7.2 \mathrm{~b}$. This demonstrates the possibility of implementing a 15 ns coincidence timing window without losing true coincidence data.

\section{Septum magnet field}

The test run demonstrated that the septum magnet provides the required field uniformity when used to bend $1.13 \mathrm{GeV}$ particles to $5^{\circ}$. The highest-energy configuration for APEX (4.46 GeV) requires bending of $2.23 \mathrm{GeV}$ particles to $5.5^{\circ}$. A new septum magnet is currently being designed for Hall A experiments and will provide a uniform magnetic field under such a configuration [34]. 

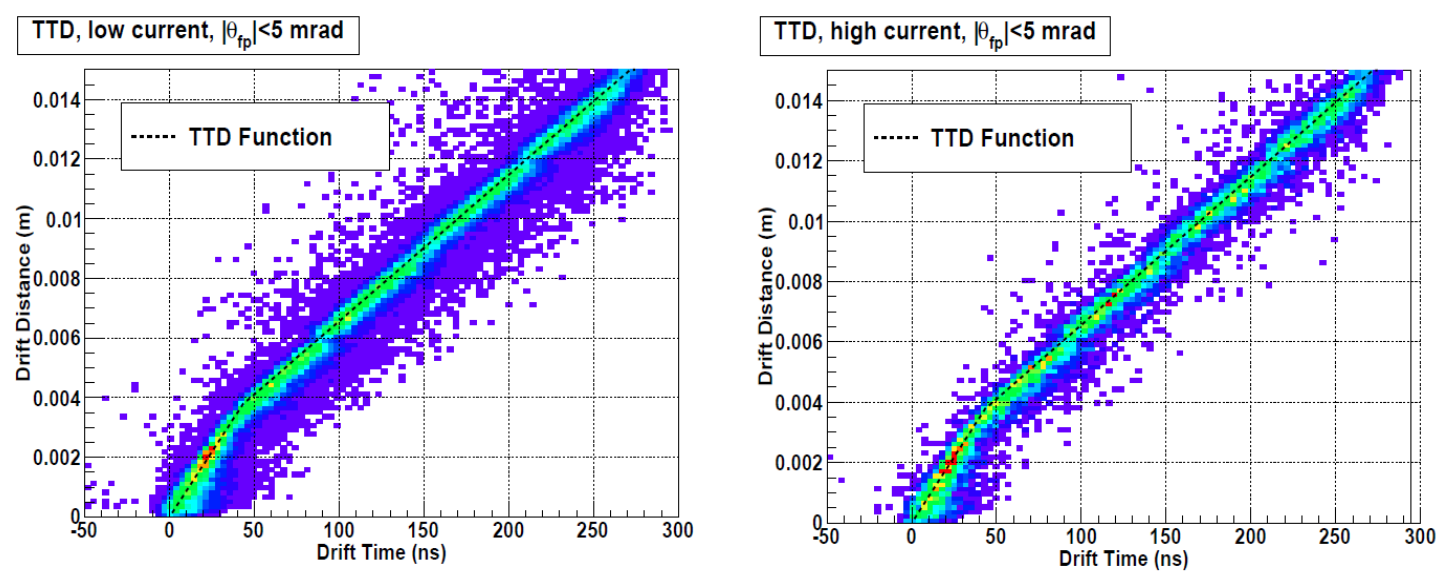

FIG. 7.17: The VDC drift time-to-distance calibration. There are no serious differences between high and low rate data.

\section{Backgrounds}

The $A^{\prime}$ will appear as a narrow resonance on top of a smooth QED background distribution. The QED background will consist primarily of $e^{+} e^{-}$pairs produced through bremsstrahlung radiation of virtual photons. The signal to background ratio is not reducible but defined by the ratio of coupling constants, $\alpha^{\prime} / \alpha_{\mathrm{fs}}$. Any additional contributions to the background, physics or accidental, lead to a relatively small loss in the experiment's sensitivity as long as their rates are only a fraction of the QED pair production rate. The amount of additional background present in the final test run data sample was calculated in order to understand its impact on the sensitivity of the $A^{\prime}$ search.

The GC detectors in both spectrometers were used to determine how much 

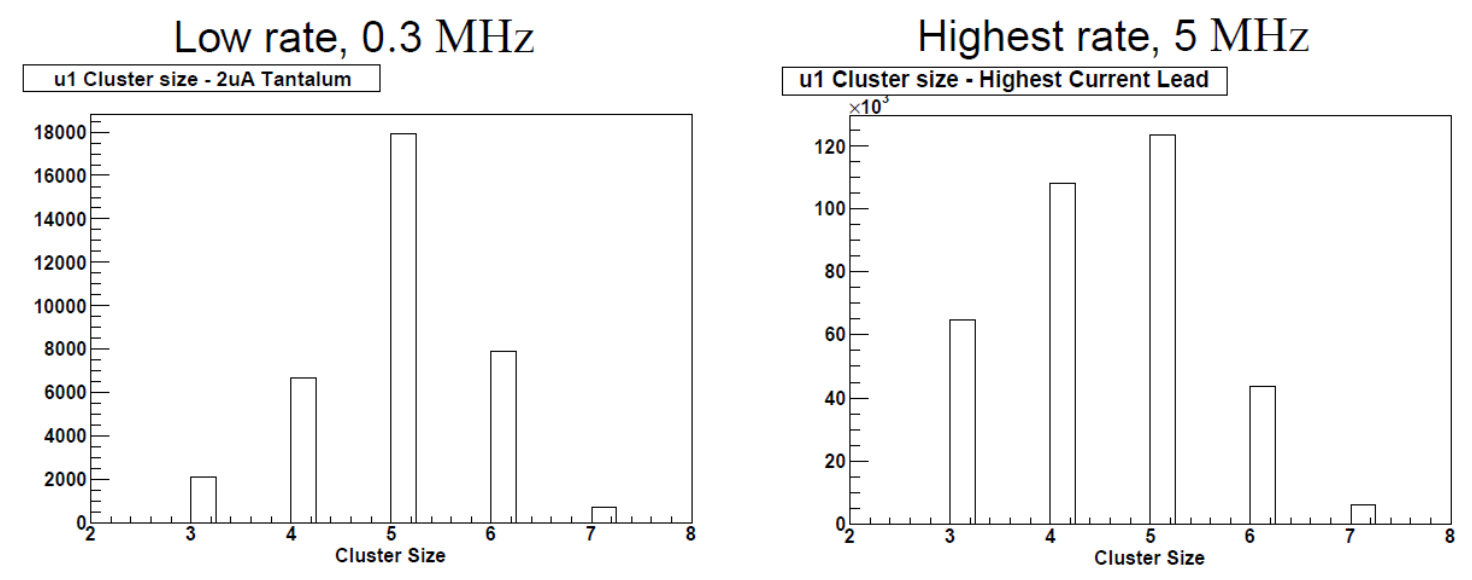

FIG. 7.18: Cluster sizes for low and high rate data. We observe a reduction in cluster size for high rate data due to reduced efficiency.

meson background was present in the final data sample. Events with a GC amplitude (sum of all 10 PMTs) less than 100 ADC counts are considered to be meson background. The final data sample contains only $0.9 \%$ meson background contamination, the majority of which is made of $e^{+} \pi^{-}$events. The accidental background was estimated using the coincidence timing spectrum shown in Fig. 7.2b. True coincidence events were selected by making a $12.5 \mathrm{~ns}$ cut centered about the peak. Events outside of this $12.5 \mathrm{~ns}$ window are considered to be accidentals. Using this coincidence time spectrum we find that $7.4 \%$ of the final event sample is made of accidental coincidence events. 


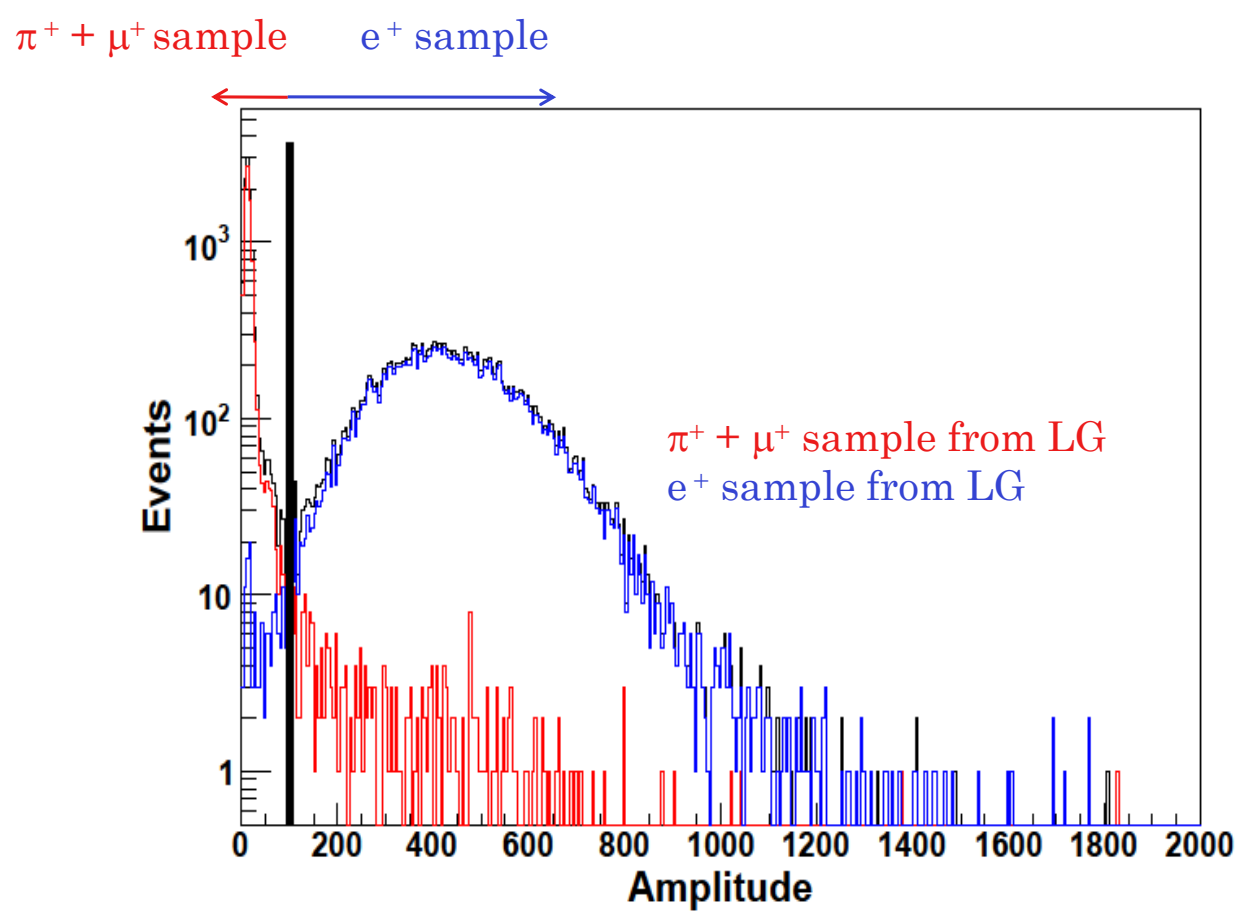

FIG. 7.19: The Right-HRS gas Cherenkov amplitude spectrum at a track rate of 750 $\mathrm{kHz}$. At this rate the pion rejection factor is 30 , which is sufficient for the reduction of the DAQ rate. 


\section{CHAPTER 8}

\section{Resonance Search and Results}

The analysis of the APEX test run data yields an invariant mass spectrum of $e^{+} e^{-}$pairs. This mass spectrum provides the starting point for the $A^{\prime} \rightarrow e^{+} e^{-}$ search. The next steps are to search the mass spectrum for a resonance and, if a resonance is not found, set an exclusion limit on the coupling $\alpha^{\prime}$. In this chapter we will describe the procedure used to search for peaks in the spectrum and quantify both the significance of any observed peaks and the exclusion power of the experiment. The entire APEX collaboration contributed to the resonance search analysis. The final results of the search will be presented at the end of the chapter.

\subsection{Searching for a resonance}

An $A^{\prime}$ signal would appear as a Gaussian peak on top of a polynomial background distribution. The signal would have an unknown height and width $\sigma$ equal to the experimental mass resolution. The general idea behind the $A^{\prime}$ resonance search is to scan the entire $e^{+} e^{-}$invariant mass spectrum searching for a significant number of signal events at each mass hypothesis. The search is performed by constructing a fixed size window centered around the mass hypothesis $m$. The data in this window 
is fit with a polynomial plus a Gaussian centered at $m$ with a width $\sigma$ equal to the experimental mass resolution. The polynomial coefficients and the normalization of the Gaussian are free parameters chosen to maximize the Poisson likelihood of the data (see Sec. ??).

The significance of a resonance can be formulated in terms of the probability that the resonance could be observed by accident. In other words, the probability that such a resonance can be observed due to statistical fluctuations. To determine this probability a series of pseudo-experiments are performed on background only Monte Carlo data sets. These data sets are independently generated based on the toy function described in Sec. 8.1.1. To quantify the significance of an observed signal we use a quantity known as a $p$-value. The $p$-value is the probability that an experimental observation agrees with the null hypothesis (no signal present). Observing a large signal would have small agreement with the null hypothesis, and thus give a small $p$-value. For example, if an observed signal has a $p$-value $p_{\text {obs }}$, and a $p$-value that is less than or equal to $p_{\text {obs }}$ is observed in $50 \%$ of the background only pseudo-data sets, then there is a $50 \%$ chance of accidentally producing such a signal through statistical fluctuations and it should not be considered as evidence of new physics.

Often in physics the statistical significance of a result is given in terms of the standard deviation $\sigma$ of a normal distribution. A normal distribution and integer multiples of $\sigma$ are shown in Fig. 8.1. The number of sigmas measures the probability of observing the same result by chance. This probability is obtained by integrating the normal distribution from $n \sigma$ to infinity. For example, a $3 \sigma$ signal would have a $1.35 \times 10^{-3}$ chance of occurring due to statistical fluctuations. So in our case, a $3 \sigma$ evidence would require that such an observed signal only occurs with a frequency of $1.35 \times 10^{-3}$ in the background only pseudo-data sets. 


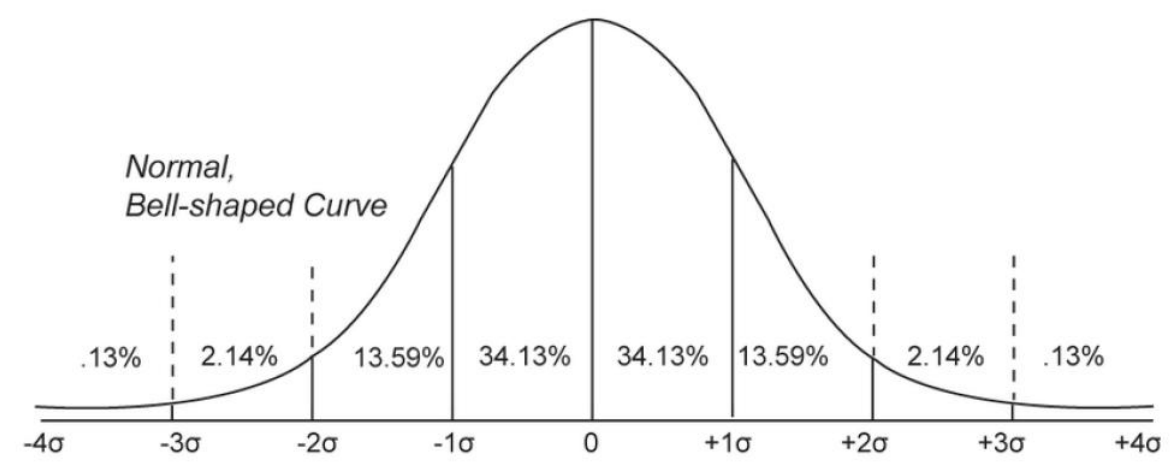

FIG. 8.1: A normal distribution with integer multiples of the standard deviation $\sigma$ shown. The statistical significance of a result is often given in terms of the number of standard deviations, $n \sigma$. The probability of producing a result by chance is found by integrating the normal distribution from $n \sigma$ to infinity. These probabilities are shown up to $3 \sigma$.

\subsubsection{Pseudo-data sets}

The pseudo-data sets were generated from the "toy function"

$$
\frac{(170-m)^{2}(265-m)^{2}}{m^{4}}
$$

between 171 and $264 \mathrm{MeV}$ (Fig. 8.2). Each bin of a data set was filled according to independent Poisson distributions with expectation values given by the function above scaled to provide a total of $7 \times 10^{5}$ events. Pseudo-data sets with signals were also created using the same procedure with signal events generated according to a Gaussian probability distribution function (PDF).

\subsubsection{Profile likelihood ratio}

For a fixed $A^{\prime}$ mass $m_{A^{\prime}}$, the distribution of events can be modeled using the probability distribution

$$
P\left(m_{e^{+} e^{-}}\right)=\frac{1}{S+B}\left(S \cdot \mathrm{N}\left(m_{e^{+} e^{-}} \mid m_{A^{\prime}}, \sigma\right)+B \cdot \operatorname{Polynomial}\left(m_{e^{+} e^{-}}, a_{i}\right)\right),
$$




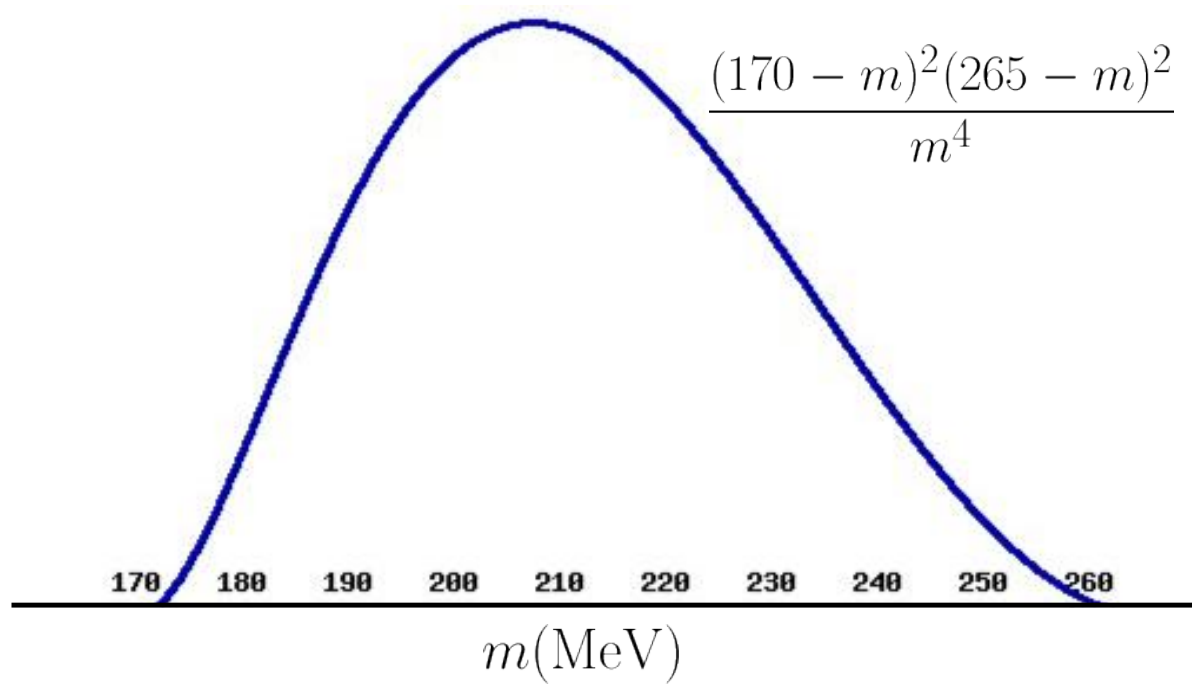

FIG. 8.2: The "toy function" used to generate pseudo-data sets.

where $m_{e^{+} e^{-}}$is the invariant mass of the $e^{+} e^{-}$pair, $S$ is the number of signal events, $B$ is the number of background events, $\mathrm{N}$ is a normal (Gaussian) probability distribution, and the background shape is given by a polynomial with coefficients $a_{i}$.

This probability function becomes a likelihood function, $L$, as a function of the model parameters. To test a hypothesized value for $S$ against alternatives, we use the profile likelihood ratio (PLR) 35]

$$
\lambda(S)=\frac{L\left(S, \hat{\hat{B}}, \hat{\hat{a}}_{i}\right)}{L\left(\hat{S}, \hat{B}, \hat{a}_{i}\right)} .
$$

In the numerator, $\hat{\hat{B}}$ and $\hat{\hat{a}}_{i}$ are the values of $B$ and $a_{i}$ that maximize $L$ for the assumed $S$. In other words, $\hat{\hat{B}}$ and $\hat{\hat{a}}_{i}$ are conditional Maximum Likelihood Estimators (MLEs) and consequently are functions of $S$ itself. The denominator, instead, is maximized to best fit the data without any constraints on $S$, thus $\hat{S}, \hat{B}$, and $\hat{a}_{i}$ are the unconditional MLEs. When the hypothesized $S$ coincides with $\hat{S}$, the PLR goes to 1, thus showing great compatibility between the data and the hypoth- 
esis. If $S$ does not agree with $\hat{S}$, the PLR goes to 0 , thus showing a high degree of incompatibility between the data and hypothesis.

The Wilks' theorem states that under the null hypothesis, or $S=0$, the loglikelihood ratio $t=-2 \ln \lambda(S)$ is distributed according to a $\chi^{2}$ function with the number of degrees of freedom equal to the number of parameters of interest, which in our case is the one parameter $S$ [36]. Because of its $\chi^{2}$ distribution under the null hypothesis, the quantity $t$ can be used as a test statistic to either claim discovery of a new signal or to put an upper limit on the case of the absence of a signal.

For example, if we want to establish an upper limit at $90 \%$ confidence, a threshold is set so that the integral of the null hypothesis probability distribution function (PDF) from 0 to the threshold is 0.90. Then for a given experimental outcome, the value of $t$ is calculated for the observed data. If $t$ is found to be equal to or greater than the threshold, the presence of a signal is excluded at a level equal or greater than $95 \%$.

The observed test statistic $t_{o b s}$ can be translated into a $p$-value using

$$
p=\int_{t_{o b s}}^{\infty} f(t) d t
$$

where $f(t)$ is the probability distribution function of $t$. Fig. 8.3 illustrates the relation between the $p$-value obtained from the observed $t$ as well as its relation to the significance $Z$.

\subsubsection{The Look Elsewhere Effect}

When testing the background only (null) hypothesis it is possible for a large statistical fluctuation to mimic a signal. Furthermore, when scanning a wide mass range for a resonance it is possible for such statistical fluctuations to occur anywhere within the mass range. Thus the probability of observing an accidental signal 


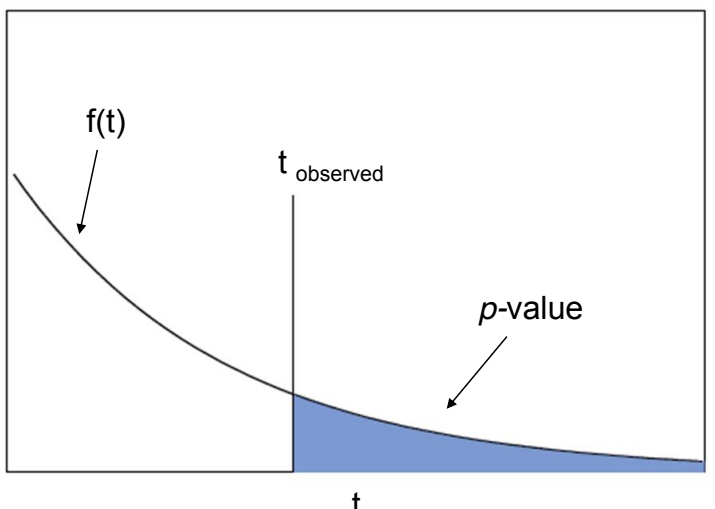

(a)

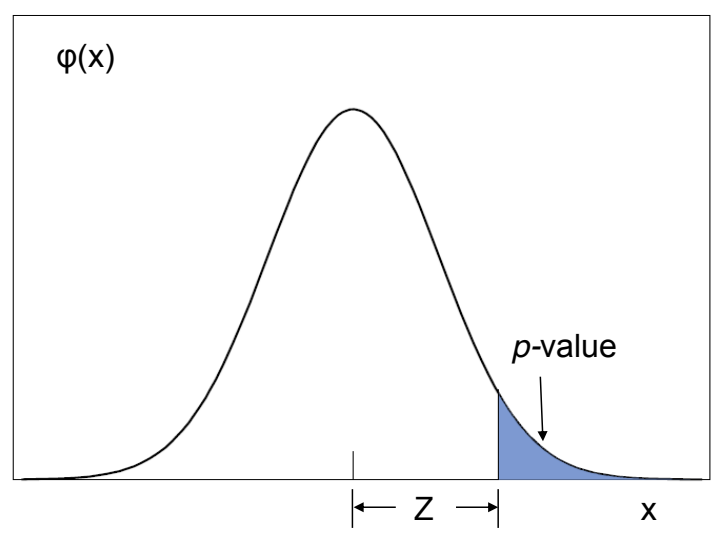

(b)

FIG. 8.3: (A) The relation between the $p$-value and PDF of the test statistic $f(t)$. The $p$-value is obtained from the observed value of the test statistic $t_{\text {observed }}$ (B) The standard normal distribution $\Phi(x)$ showing the relation between the $p$-value and the significance $Z$.

increases significantly. This phenomenon is known as the "Look Elsewhere Effect" (LEE).

Take, for example, a mass range consisting of a single bin. If we perform 100 pseudo-experiments and find $S$ signal events in 10 of them, then there is a $10 \%$ chance of observing such a signal from statistical fluctuations. Now let's take a mass range consisting of 10 bins. For simplicity, let's assume the probability of observing $S$ signal events in each of the 10 bins is $10 \%$. The probability of observing an accidental signal in our 10 bin spectrum would be $100 \%$.

One simple way of accounting for the LEE is to scale the lowest $p$-value obtained from the data by a "trials factor", which is the ratio between the probability of observing a signal at some fixed mass point to the probability of observing it anywhere in the range. This scaling can be quantitatively expressed as

$$
p \rightarrow p \times \frac{\text { massrange }}{\text { massresolution }}
$$

This is equivalent to requiring a smaller threshold for the uncorrected $p$-value when 
claiming evidence of new physics. In reality, the "trials factor" is only a first order correction and is too crude for determining a threshold for the smallest $p$-value with good accuracy.

Another solution is to run many pseudo-experiments on the background only Monte Carlo data sets described in Sec. 8.1.1 and find for each one the fluctuation resulting in the lowest $p$-value. For $3 \sigma$ evidence of an $A^{\prime}$ signal, a $p$-value less than or equal to the threshold should only be observed with frequency $1.35 \times 10^{-3}$. Using this method would yield the right answer, however obtaining the correct threshold with any accuracy would require a very large number of pseudo-experiments. Instead, the method described in [37] was used to obtain a threshold. This method only requires a modest number of pseudo-experiments. The lowest $p$-value from each pseudoexperiment is ranked, and the estimated quantile is obtained by dividing the rank by the total number of pseudo-experiments performed plus one. The $p$-values are then plotted versus their respective quantile. Fig. 8.4 shows an example of such a plot containing data from 7500 pseudo-experiments. In this example the $p$-value for $3 \sigma$ significance is around $10^{-5}$.

\subsubsection{Search parameters}

Before performing the resonance search some search parameters must be optimized. These parameters include the size of the invariant mass binning, the fitting window size, and the order of the polynomial in the fit function. The search parameters were tested on the pseudo-data sets described in Sec. 8.2 in order to find values for the parameters that maximize the sensitivity of the search while also minimizing systematic pulls. The parameters were also tested on a $10 \%$ sample of the experimental data that was scaled up in order to avoid potential bias. 


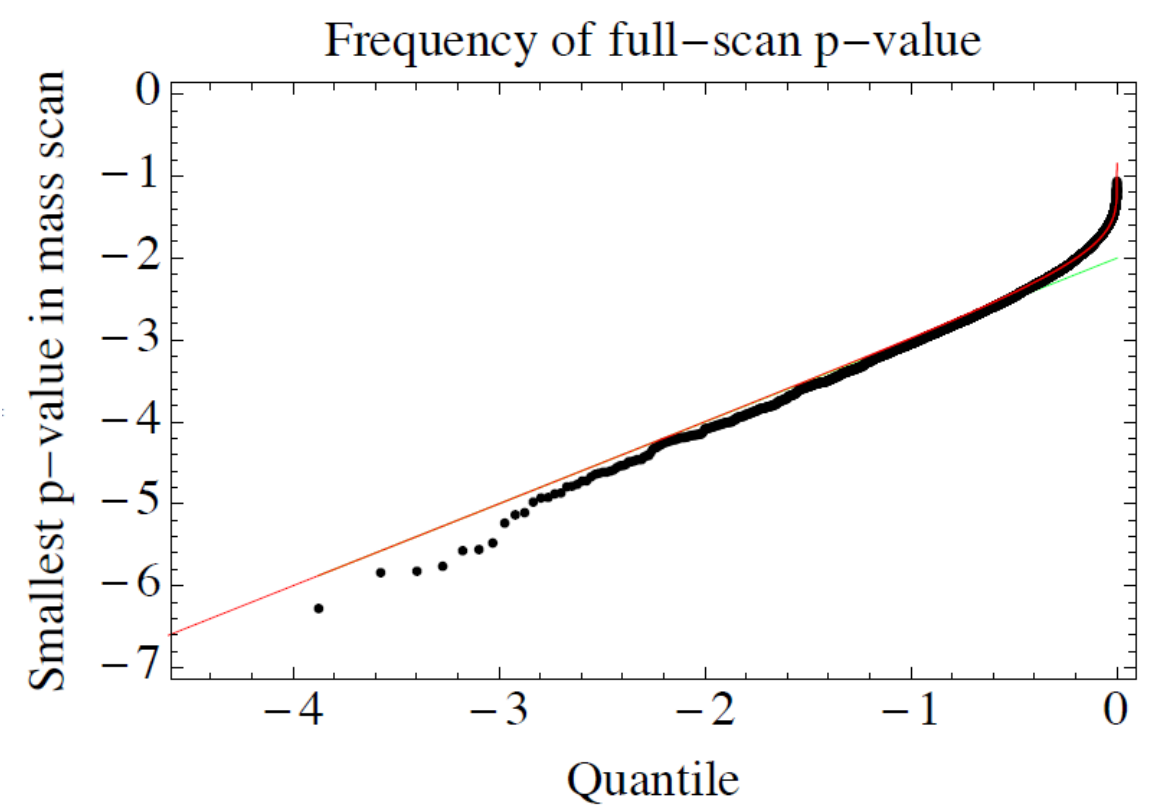

FIG. 8.4: An example of limit setting on the lowest observed $p$-value. Full mass scans were performed on 7500 pseudo-data sets. The lowest $p$-value was obtained from each mass scan and ranked. The quantile of each pseudo-experiment was calculated by dividing the rank by the (total number of pseudo-experiments performed +1 ). This plot shows the $p$-value vs. quantile for all 7500 mass scans. The numbers on the axes are exponents. From this plot we find that a signal with $3 \sigma$ significance, or occurring with a frequency of $1.35 \times 10^{-3}$ from statistical fluctuations, would require a $p$-value of $\sim 10^{-5}$.

\section{Mass binning}

Ideally the peak search would be performed using an unbinned mass spectrum. Due to the large amount of statistics, however, this would be intractably time consuming. A binned analysis is much more manageable and any systematic pulls due to choice of statistical tool (ROOT, Mathematica, etc.) can be made negligible by choosing small enough bins.

The systematic pull was calculated for pseudo-data sets using

$$
\text { Pull }=\frac{S_{\text {bestfit }}-S_{\text {inserted }}}{S_{\text {error,fit }}} .
$$



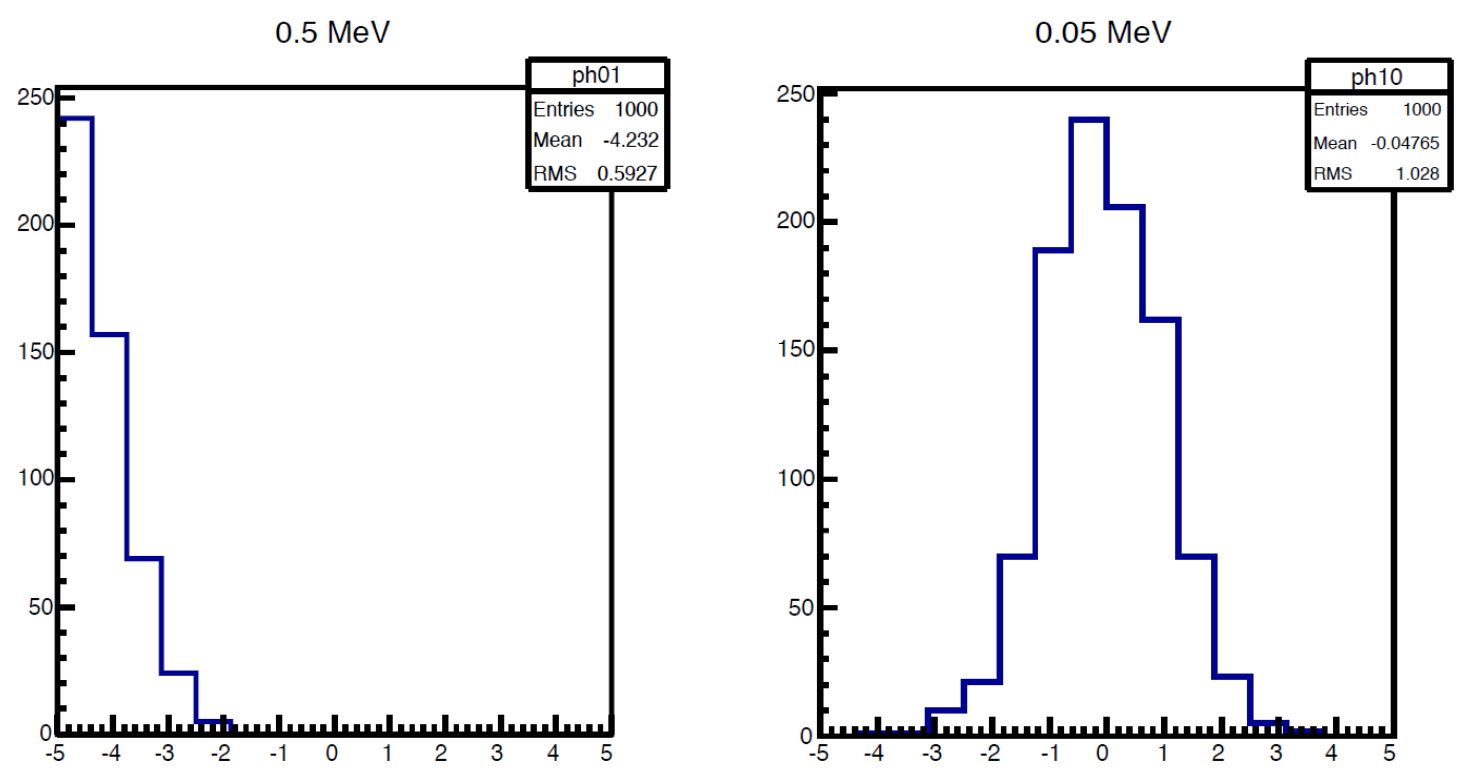

FIG. 8.5: Pulls generated for pseudo-data sets when $S_{\text {inserted }}=0$. The pull is calculated using Pull $=\frac{S_{\text {bestfit }}-S_{\text {inserted }}}{S_{\text {error,fit }}}$. An average pull of -4.232 is generated for $0.5 \mathrm{MeV}$ binning (left) and -0.048 for $0.05 \mathrm{MeV}$ binning (right).

The pulls were calculated for several different bin sizes. The pull distributions for 0.5 $\mathrm{MeV}$ and $0.05 \mathrm{MeV}$ bin sizes are shown in Fig. 8.5. We found that when $S_{\text {inserted }}=0$ the average pull generated using a bin size of $0.05 \mathrm{MeV}$ was $\sim 100$ times smaller than when using a bin size of $0.5 \mathrm{MeV}$. A bin size of $0.05 \mathrm{MeV}$ was used in the final analysis.

\section{Polynomial order and window size}

To search for the $A^{\prime}$ a polynomial background model plus a Gaussian signal is fit to a window centered about each candidate $A^{\prime}$ mass. The uncertainty in the polynomial coefficients corresponds to the uncertainty in the shape of the background 


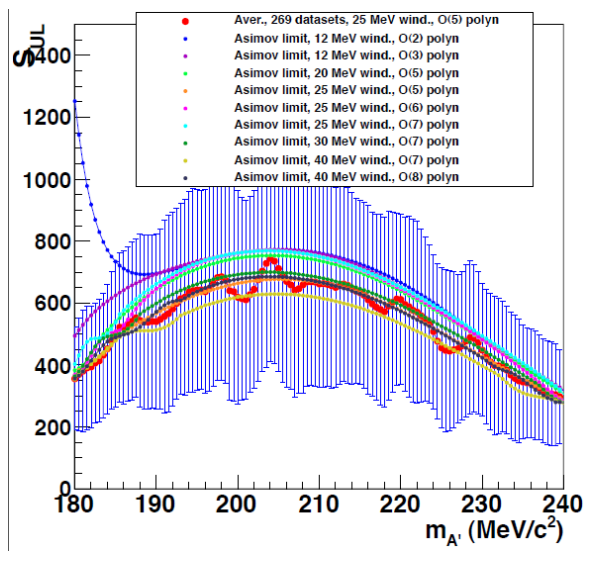

(a)

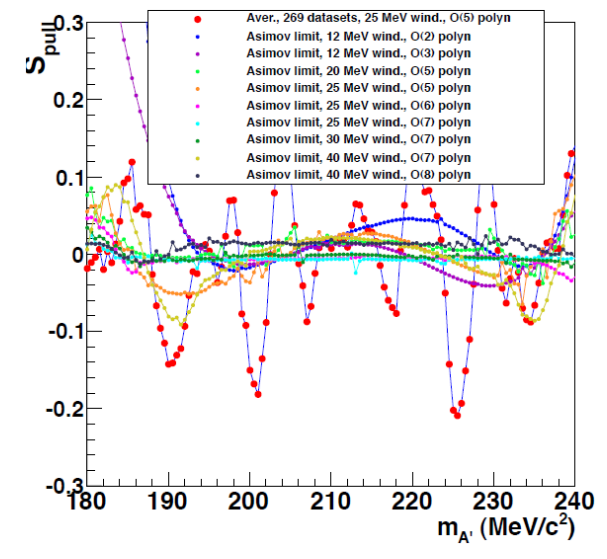

(b)

FIG. 8.6: Different combinations of polynomial order and window size are tested in order to optimize between sensitivity in $S$ and minimal pull. (A) shows the upper limit on the number of signal events $S$ allowed for the null hypothesis to hold true. (B) shows the average pulls generated by the different combinations.

model. To optimize the sensitivity of the resonance search the uncertainty in the shape of the background model must be minimized. Extensive tests were done on pseudo-data sets to determine which window size and order of polynomial optimized the sensitivity of the search while also minimizing systematic pulls.

Fig. 8.6 shows results of tests that were done using several different values of polynomial order and window size. Fig. 8.6a demonstrates the upper limit on the number of signal events allowed for the null hypothesis to hold true. Fig. 8.6b shows the systematic pulls generated by each test case. It was found that a $30.5 \mathrm{MeV}$ window with 7 th order polynomial (dark green line) optimize between sensitivity in $S$ and minimal pull. The window is centered about each mass candidate, except for masses within $15 \mathrm{MeV}$ of the edge of the spectrum, for which a window of equal size touching the boundary is used. The fit is repeated across the mass spectrum in steps of $0.25 \mathrm{MeV}$. 


\subsubsection{Results}

The results of the resonance search show no evidence of an $A^{\prime}$ signal in the mass range of $175-250 \mathrm{MeV}$. The most significant signal found $224.5 \mathrm{MeV}$ has a local $p$-value of $0.6 \%$ (see Fig. 8.7). After correcting for the LEE the associated global $p$-value is $40 \%$, meaning that $40 \%$ of the background only pseudo-experiments resulted in more significant signals due to statistical fluctuations.

\subsection{Setting limits on $\alpha^{\prime} / \alpha_{\mathrm{fs}}$}

The second part of performing the $A^{\prime}$ search is setting a limit on the coupling $\alpha^{\prime}$. The goal is to translate the upper limit on the number of signal events $S$ into a limit on $\alpha^{\prime}$. If $\alpha^{\prime}$ is extremely small, the $A^{\prime}$ production cross section will also be small and the number of observed signal events will be reduced. Statistical fluctuations may then mask the presence of a true $A^{\prime}$ signal. To search for $A^{\prime}$ s with smaller couplings we must increase the amount statistics collected during the experiment, thus reducing the size of the statistical fluctuations. By collecting more statistics we extend the experimental reach to smaller values of $\alpha^{\prime}$. A large number of signal events observed at $m_{\text {hyp }}$ might not be large enough to show evidence of an $A^{\prime}$ signal if there is a significant probability of producing such a signal through statistical fluctuations. This doesn't mean that an $A^{\prime}$ with mass $m_{\text {hyp }}$ does not exist. The $A^{\prime}$ could have a very small coupling, thus masking the "true" signal with statistical fluctuations. In such a case the number of observed signal events translates into a limit on $\alpha^{\prime}$ that can be excluded. The existence of an $A^{\prime}$ with $\alpha^{\prime}$ smaller than this limit would not be excluded.

As discussed in Sec. 4.2 we use a ratio method that normalizes $A^{\prime}$ production to the measured QED trident rate to minimize systematic uncertainty from acceptance 
and trigger efficiencies. Using this method we will set an upper limit on $\alpha^{\prime} / \alpha_{\mathrm{fs}}$ using the equation

$$
\left(\frac{\alpha^{\prime}}{\alpha_{\mathrm{fs}}}\right)_{\max }=\left(\frac{S_{\mathrm{max}} / m_{A^{\prime}}}{f \dot{B} / \delta m}\right) \times\left(\frac{2 N_{\mathrm{eff}} \alpha_{\mathrm{fs}}}{3 \pi}\right),
$$

where $N_{\text {eff }}$ is the number of possible decay channels $\left(N_{\text {eff }}=1\right.$ for $m_{A^{\prime}}<2 m_{\mu}$, and increases to $\simeq 1.6$ at $\left.m_{A^{\prime}} \simeq 250 \mathrm{MeV}\right), f$ is the ratio of the radiative-only cross section to the full trident cross section (varies linearly from 0.21 to 0.25 across the APEX mass range), $S_{\max }$ is the upper limit on the number of signal events observed at $m_{A^{\prime}}$, and $B / \delta m$ is the number trident background events observed per unit mass evaluated in a $1 \mathrm{MeV}$ range around $m_{A^{\prime}}$.

\subsubsection{Setting limits on $S$}

The number of observed signal events $S$ is determined using the PLR method described in Sec. 8.1.2. This method finds the value of $S$ that best fits the data. The log-likelihood ratio is used to calculate the corresponding $p$-value, which is then corrected for the LEE. If the $p$-value does not show evidence of an $A^{\prime}$ signal, then we set a limit on $\alpha^{\prime}$ using the upper limit of $S$. The $2 \sigma$ (90\% confidence) upper limit of $S$ is derived by setting the $p$-value to 0.1 and then inverting the PLR to solve for $S_{\max }$. The LEE does not need to be accounted for when deriving $S_{\max }$.

The statistical fluctuations that produce artificial signals can also have the opposite effect, resulting in flat or negative signals. Regions of the mass spectrum where the best fit results in a value of $S$ that is equal to or even less than zero would result in the exclusion of all possible values of $\alpha^{\prime}$ according to Eq. 8.7. As a result, these regions lack sensitivity in the search for a signal. To avoid completely excluding such regions we use a $50 \%$ power-constrained limit on $S$ [38]. For each mass candidate there is an associated $S_{\text {median, }}$ which is the median value of the $2 \sigma$ limit of signal events observed for all pseudo-experiments. If $S_{\max }<S_{\text {median }}$ 
(the observed upper limit is less than the pseudo-experiment median upper limit) then the resonance search is said to have insufficient signal sensitivity for that mass candidate. In such a case, $S_{\text {median }}$ is used instead of $S_{\max }$.

\subsubsection{Results}

The middle section of Fig. 8.7 shows the $2 \sigma$ upper bound on the absolute yield of $A^{\prime} \rightarrow e^{+} e^{-}$signal events across the $A^{\prime}$ mass spectrum. The red line shows the best fit of $S$. The shaded grey region denotes the $2 \sigma$ upper limit with $50 \%$ powerconstraint. The expected limit $\left(S_{\text {median }}\right)$ is denoted by the dashed grey line. The solid (dotted) blue line shows the $2 \sigma$ limit when it is above (below) the expected limit. For comparison, the dot-dashed line shows the expected limit if the background shape were known exactly, i.e. if the polynomial coefficients were fixed. This illustrates the contribution of statistical uncertainty to the expected limit.

The resulting $2 \sigma$ limit on $\alpha^{\prime} / \alpha_{\mathrm{fs}}$ is shown in blue in Fig. 8.8. The small gaps are associated with the larger signal excesses that were observed in the data. Also shown are existing $2 \sigma$ limits from the muon anomalous magnetic moment $a_{\mu}$ (fine hatched) [14], KLOE (solid gray) [39], the result reported by Mainz (green) [40], and an estimate using a BaBar result (wide hatched) [15]. The full APEX experiment will roughly cover the entire area of the plot. 


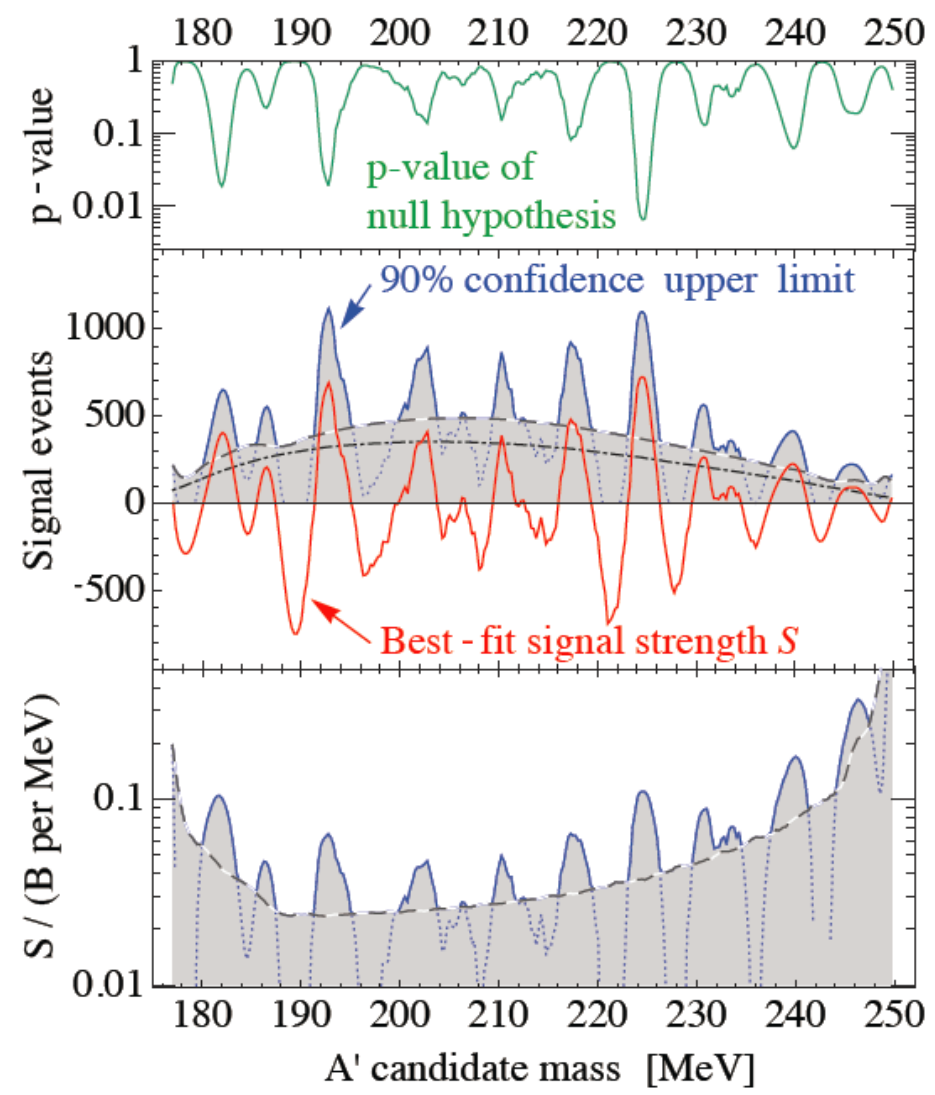

FIG. 8.7: Top: Local $p$-value versus $A^{\prime}$ mass. Middle: The $2 \sigma$ upper limit on signal events. The shaded grey region shows the $50 \%$ power-constrained region. The red line denotes the best fit on the number of signal events. The expected limit is shown by the dashed line. The blue solid (dotted) line denotes the $2 \sigma$ upper limit when it falls above (below) the expected limit. To illustrate the contribution from statistical fluctuations, the dot-dashed line shows the expected limit if the background shape were known exactly. Bottom: The $50 \%$ power-constrained $2 \sigma$ upper limit and expected limits as above, but shown as the ratio of signal events to QED background. 


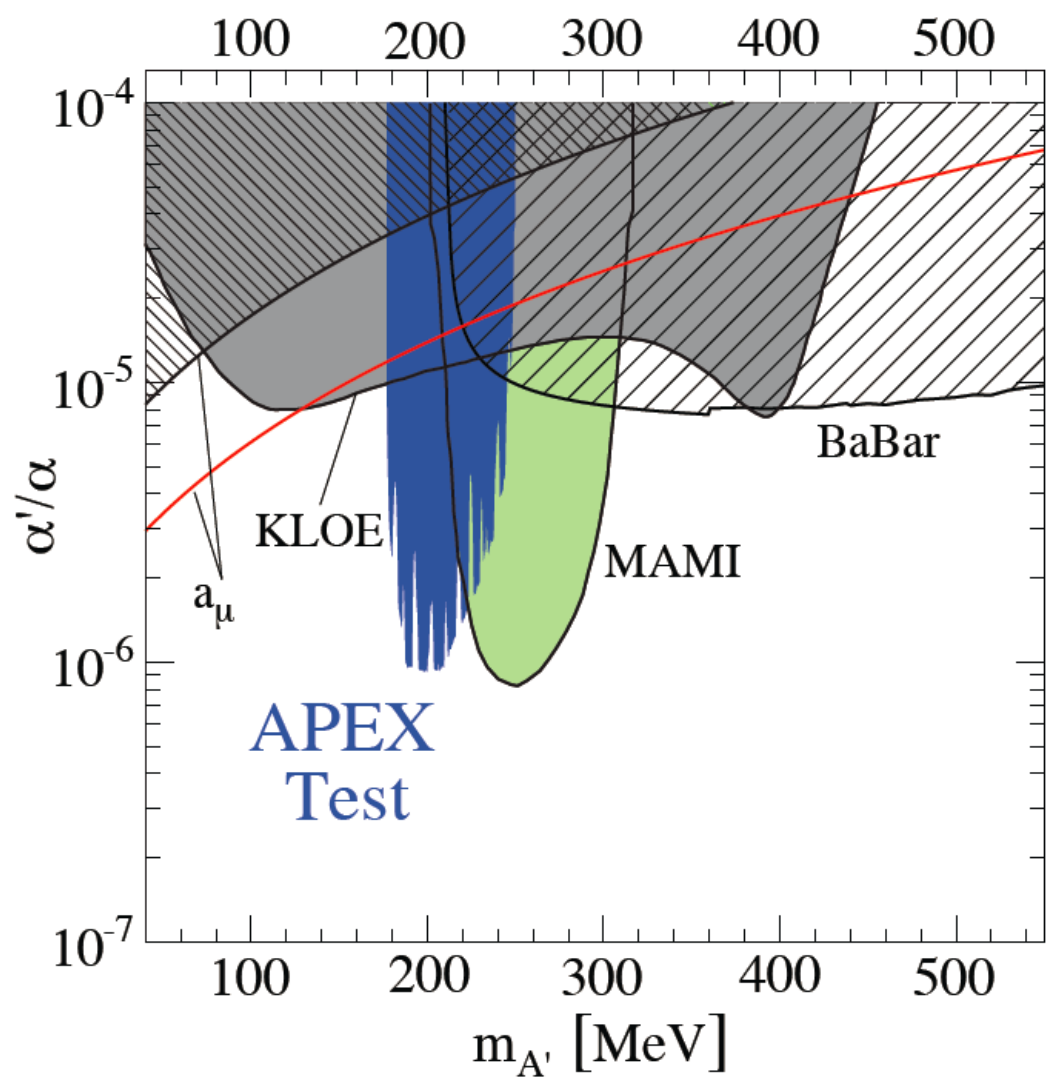

FIG. 8.8: The $2 \sigma$ upper limit on $\alpha^{\prime} / \alpha_{\mathrm{fs}}$ versus $A^{\prime}$ mass for the APEX test run is shown in blue. Also shown are existing $2 \sigma$ limits from the muon anomalous magnetic moment $a_{\mu}$ (fine hatched) [14], KLOE (solid gray) [39], the result reported by Mainz (green) [40], and an estimate using a BaBar result (wide hatched) [15]. 


\section{CHAPTER 9}

\section{Conclusion}




\section{BIBLIOGRAPHY}

[1] C. W. Leemann, D. R. Douglas, and G. A. Krafft, Annu. Rev. Nucl. Sci. 51, $413(2001)$.

[2] K. Unser, Nucl. Sci. 28, 2344 (1981).

[3] H. A. Collaboration, Nucl. Phys. A522, 294 (2004).

[4] W. Barry, Tech. Rep. JLab-TN-91-087, Jefferson Lab (1991).

[5] K. Fissum et al., Nucl. Phys. A474, 108 (2001).

[6] H. A. Collaboration, Tech. Rep., Jefferson Lab (2008), URL hallaweb.jlab. org/experiment/E05-102/e05-102/docs/osp_4.pdf.

[7] W. A. Watson, J. Chen, G. Heyes, E. Jastrzembski, and D. Quarrie, Tech. Rep., Jefferson Lab (1993).

[8] J. D. Bjorken et al., Tech. Rep., Jefferson Lab (2010), URL http://hallaweb. jlab.org/collab/PAC/PAC37/E12-10-009-APEX.pdf.

[9] R. Essig, P. Schuster, and N. Toro, Phys. Rev. Lett. D80, 015003 (2009).

[10] J. D. Bjorken, R. Essig, P. Schuster, and N. Toro, Phys. Rev. Lett. D80, 075018 (2009).

[11] K. J. Kim and Y. Tsai, Phys. Rev. D 8, 3109 (1973). 
[12] J. Alwall, P. Demin, S. de Visscher, R. Frederix, M. Herquet, et al., J. High Energy Phys. 0709, 028 (2007).

[13] S. Abrahamyan et al., arXiv:1108.2750 [hep-ex] (2011).

[14] M. Pospelov, Phys. Rev. Lett. D80, 095002 (2009).

[15] B. Aubert et al. (BABAR Collaboration), in 2009 Aspen Winter Conference on Astronomy: THIRTY YEARS OF MAGNETARS: NEW FRONTIERS (2009).

[16] J. Bjorken, S. Ecklund, W. Nelson, A. Abashian, C. Church, et al., Phys. Rev. Lett. D38, 3375 (1988).

[17] E. Riordan, M. Krasny, K. Lang, P. De Barbaro, A. Bodek, et al., Phys. Rev. Lett. 59, 755 (1987).

[18] A. Bross, M. Crisler, S. H. Pordes, J. Volk, S. Errede, et al., Phys. Rev. Lett. 67, 2942 (1991).

[19] J. D. Bjorken, R. Essig, P. Schuster, and N. Toro, Phys. Rev. Lett. D80, 075018 (2009).

[20] S. Abrahamyan et al., Tech. Rep., Jefferson Lab (2010), URL http://www. jlab.org/conferences/boson2010/program.html.

[21] I. Rachek et al., Tech. Rep., Jefferson Lab (2007).

[22] I. Rachek and B. Wojtsekhowski, Tech. Rep. JLab-TN-11-017, Jefferson Lab (2011).

[23] H. E. Montgomery et al., Tech. Rep., Jefferson Lab (2010), URL http://www . jlab.org/exp_prog/PACpage/PAC35/PAC35_report.pdf. 
[24] S. Abrahamyan et al. (PREX Collaboration), Phys. Rev. Lett. 108, 112502 (2012), URL http://link .aps .org/doi/10.1103/PhysRevLett.108. 112502 .

[25] S. Riordan, Ph.D. thesis, Carnegie Mellon University (2008).

[26] M. Mihovilovic, Ph.D. thesis, University of Ljubljana (2012).

[27] N. Liyanage, Tech. Rep. JLAB-TN-02-012, Jefferson Lab (2002).

[28] J. Huang, Ph.D. thesis (Massachusetts Institute of Technology).

[29] O. Hanson, Root/c++ analyzer for hall a, URL http://hallaweb.jlab.org/ podd/.

[30] R. Brun, ROOT, an object-oriented data analysis framework, URL http:// root.cern.ch/drupal/.

[31] A. Orsborn, Tech. Rep. JLAB-TN-05-069, Jefferson Lab (2005).

[32] (2010), URL http://conferences.jlab.org/boson2010/program.html.

[33] Y. Qiang, Ph.D. thesis (????).

[34] B. Wojtsekhowski, Apex: A search for dark photons in hall a (e1210-009), URL http://hallaweb.jlab.org/collab/meeting/2012-winter/ talks/Tuesday\%20Afternoon/Wojtsekhowski_APEX.pdf.

[35] G. Ranucci, Nuclear Instruments and Methods in Physics Research A 661, 77 (2012).

[36] S. S. Wilks, The Annals of Mathematical Statistics 9, 60 (1938).

[37] E. Gross and O. Vitells, European Physical Journal C 70, 525 (2010). 
[38] G. Cowan, K. Cranmer, E. Gross, and O. Vitells, arXiv:1105.3166 (2011).

[39] F. Archilli et al., arXiv:1107.2531 (2011).

[40] H. Merkel and others (A1), Phys. Rev. 106, 251802 (2011). 